Acquisitions and

Bibliographic Services Branch

395 Wellington Street

Ottawa. Ontario

K1A ON4
Bibliothèque nationale

du Canada

Direction des acquisitions et

des services bibliographiques

395, rue Wellinglon

Ottawa (Ontario) rour tile votes retereyce

Out the Notre reterences

\section{NOTICE}

\section{AVIS}

The quality of this microform is heavily dependent upon the quality of the original thesis submitted for microfilming. Every effort has been made to ensure the highest quality of reproduction possible.

If pages are missing, contact the university which granted the degree.

Some pages may have indistinct print especially if the original pages were typed with a poor typewriter ribbon or if the university sent us an inferior photocopy.

Reproduction in full or in part of this microform is governed by the Canadian Copyright Act, R.S.C. 1970, c. C-30, and subsequent amendments.
La qualité de cette microforme dépend grandement de la qualité de la thèse soumise au microfilmage. Nous avons tout fait pour assurer une qualité supérieure de reproduction.

S'il manque des pages, veuillez communiquer avec l'université qui a conféré le grade.

La qualité d'impression de certaines pages peut laisser à désirer, surtout si les pages originales ont été dactylographiées à l'aide d'un ruban usé ou si l'université nous a fait parvenir une photocopie de qualité inférieure.

La reproduction, même partielle, de cette microforme est soumise à la Loi canadienne sur le droit d'auteur, SRC 1970, c. C-30, et ses amendements subséquents. 


\title{
THE USE OF CONTEXTUAL INFORMATION BY RIGHT BRAIN-DAMAGED INDIVIDUALS \\ IN THE RESOLUTION OF AMBIGUOUS PRONOUNS
}

\author{
Carol Leonard \\ School of Communication Sciences and Disorders \\ McGill University \\ Montreal, Canada \\ January, 1994
}

\begin{abstract}
A thesis submitted to the Faculty of Graduate Studies and Research
in partial fulfillment of the requirements for the degree of Doctor of Philosophy
\end{abstract}

(c) Carol Leonard 1994 
National Library

of Canada

Acquisitions and

Bibliographic Services Branch

395 Wellington Street

Ottawa. Ontario

KIA ON4
Bibliothèque nationale

du Canada

Direction des acquisitions el

des services bibliographiques

395, rue Wellington

Ottawa (Ontatio)
Your the volresterence

Our the Notre reterence
The author has granted an irrevocable non-exclusive licence allowing the National Library of Canada to reproduce, loan, distribute or sell copies of his/her thesis by any means and in any form or format, making this thesis available to interested persons.

The author retains ownership of the copyright in his/her thesis. Neither the thesis nor substantial extracts from it may be printed or otherwise reproduced without his/her permission.
L'auteur a accordé une licence irrévocable et non exclusive permettant à la Bibliothèque nationale du Canada de reproduire, prêter, distribuer ou vendre des copies de sa thèse de quelque manière et sous quelque forme que ce soit pour mettre des exemplaires de cette thèse à la disposition des personnes intéressées.

L'auteur conserve la propriété du droit d'auteur qui protège sa thèse. Ni la thèse ni des extraits substantiels de celle-ci ne doivent être imprimés ou autrement reproduits sans son autorisation. 
THE USE OF CONTEXTUAL INFORMATION BY RIGHT BRAIN-DAMAGED INDIVIDUALS 


\begin{abstract}
Three experiments were conducted with the primary purpose of investigating the ability of right brain-damaged (RBD) individuals to use contextual information -- at the level of the single sentence, in terms of the integration of information between clauses, and at the level of a minimal discourse (i.e. two sentences) -- in the resolution of ambiguous pronouns. The investigation was extended to a group of left brain-damaged (LBD) and non brain-damaged (NBD) individuals. Four additional studies investigated and found no age effects in the use of contextual information in pronoun resolution. The results of the experiments with brain-damaged subjects were contrary to initial expectations. All three experiments were consistent in demonstrating that the RBD group was influenced by contextual information in a manner similar to that demonstrated by both the LBD and NBD groups. The results are discussed in terms of the distinction between automatic and effortful processing.
\end{abstract}


Résumé

Trois expériences furent faites dont le but principal était d'enquêter sur la capacité d'un groupe de cérébrolésés droits (CLD) à utiliser une information contextuelle -- au niveau d'une simple phrase, en fonction de l'intégration de l'information provenant de diverses propositions, et au niveau d'un texte minimal (i.e. deux phrases) -- pour la résolution de pronoms ambigus. Cette enquête fut aussi dirigée sur un groupe de cérébrolésés gauches (CLG) et sur un groupe de sujets contrôles. Quatre autres expériences ne révélèrent aucun effet de l'âge sur l'emploi de l'information contextuelle pour la résolution de pronoms. Les résultats des expériences avec les cérébrolésés furent contraires aux prévisions initiales. Les trois expériences furent uniformes à démontrer que le groupe CLD était influencé par une information contextuelle de façon semblable aux groupes CLG et contrôle. Les résultats sont discutés en fonction de la distinction entre traitement automatique et traitement conscient. 


\section{Acknowledgments}

This research was supported by a Natural Sciences and Engineering Research Council of Canada University Research Fellowship and Grant (\#URF0035061), and a Medical Research Council of Canada Grant to Dr. Gloria Waters (\#MA9671). I was supported by a fellowship from the Natural Sciences and Engineering Research Council of Canada, a McGill Major Fellowship (J.W. McConnell), and a Faculty of Medicine Internal Studentship from McGill University. The equipment used in this study was made available through a cooperative project between McGill University and IBM Canada.

Many people were involved at various stages along the way in the completion of this thesis and I am glad to have the opportunity to acknowledge their contributions now. I would first like to thank all of the individuals who participated as subjects in this research. In particular, I would like to extend my gratitude to the patients involved. They demonstrated an unselfish willingness to share their experiences with the consequences of a stroke with the hope of ultimately helping others in the future. Their interest and participation in my research were very much appreciated. As well, I would like to acknowledge the cooperation of the following hospitals in obtaining patients: Jewish General Hospital, Montreal; Jewish Rehabilitation Hospital, Montreal; Massachusetts General Hospital, Boston; Mississauga Hospital, Mississauga; Ottawa Civic Hospital, Ottawa; Ottawa General Hospital, Ottawa.

I also wish to extend a sincere thanks to the numerous people involved in the recruitment and testing of subjects, in particular Sheila Young, Jennifer Marshall, Marc Gervais, Theresa Hinz, Melanie Malus-Abramowitz, Jodi Parnass, Amy Sullivan, and Joyce Srebro. Thanks also to Glenna Waters for her help in setting up the computer programs and to Yves Parisien for translating the abstract. 
I am especially grateful to Jackie Williams. During the past year she served as the crucial link between myself and the lab. I called on her services many times and she was always ready to help, not only with the technical and administrative aspects of my thesis but also in offering moral support at just the right times. I am sure her ears are still ringing from all my calls.

Elizabeth Rochon helped me to learn the ropes in the early goings of this thesis and was always forthcoming with helpful advice. I am particularly indebted to Darla Orchard -- my statistics buddy, fellow collaborator, and good friend. We have shared in many discussions that have provoked thought and understanding and I am sure that I am a better researcher because of it.

Thank you also to the members of my committee -- David Caplan for his time, interest, and advice, and Shari Baum, who was always ready to lend an ear, offer an opinion and who was a particularly calming influence on me.

And finally, thanks to my supervisor Gloria Waters for her time and willingness to read and re-read countless versions of my thesis. I am particularly grateful to her for providing me with the necessary guidance and resources along the way, while allowing me the freedom to make some of my own choices and decisions.

My list of acknowledgments would not be complete without extending a heartfelt thanks to my family and friends, and in particular to my parents for their love and support. And last, but not least, thanks to Pat O'Shaughnessy for his love, encouragement and belief in the value of my work. 
CHAPTER 1: INTRODUCTION. .............................

1.1 Contextual influences in language processing:

The influence of context on normal language processing. .........2

The influence of context on anaphor resolution. . . . . . . . . . 10

1.2 Contextual influences on language processing by LBD individuals. . . 21

1.3 Language deficits following right brain-damage. . . . . . . . . . . 29

Narrative deficits. . . ............................ 30

Deficits in the processing of non-literal language. ............ 37

Deficits in the processing of humor. . . . . . . . . . . . . . . . 42

Possible explanations for language deficits following right

brain-damage. ............................... 43

Summary.................................. 45

Right brain-damage and pronoun resolution. ............... 46

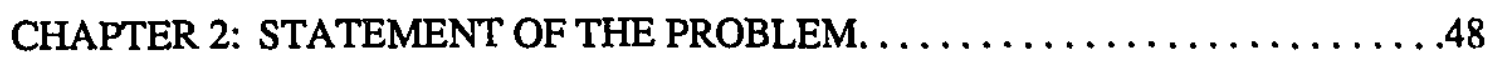

Summary of the problem......................... 51

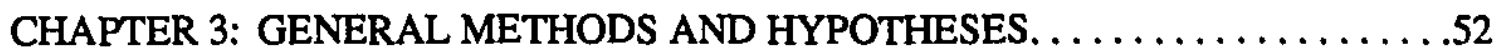

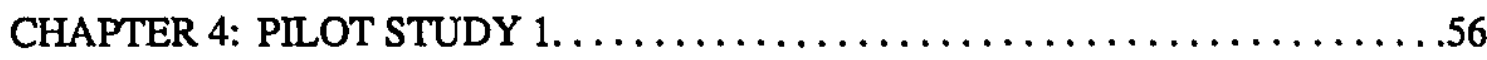

Method................................... 56

Results.................................. 57

Discussion. .................................61

CHAPTER 5: PILOT STUDIES 2a AND 2b

Pilot Study 2a...............................6 63

Method.....................................64

Results. ..................................68

Discussion............................... 72

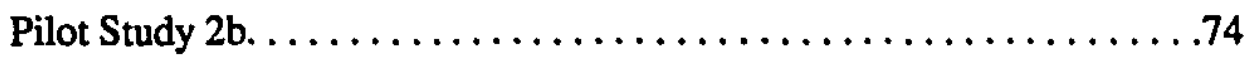

Method....................................74

Results. .................................76

Discussion...................................79

General discussion for Pilots Studies $2 \mathrm{a}$ and $2 \mathrm{~b} . \ldots \ldots \ldots \ldots \ldots \ldots . \ldots 0$ 
CHAPTER 6: PILOT STUDY $3 . \ldots \ldots \ldots \ldots \ldots \ldots \ldots \ldots \ldots \ldots \ldots \ldots \ldots \ldots \ldots$

Method. ...............................

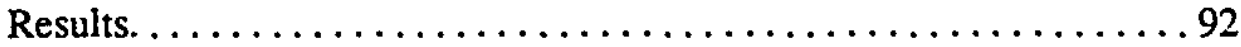

Discussion. .......................... 98

CHAPTER 7: GENERAL DISCUSSIION FOR PILOT STUDIES. . . . . . . . 102

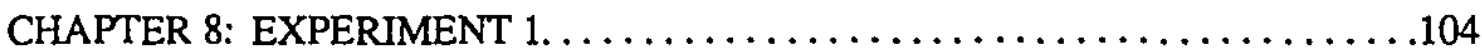

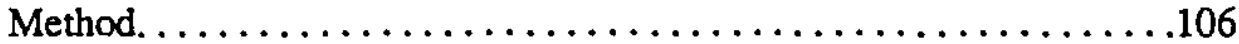

Results................................ 119

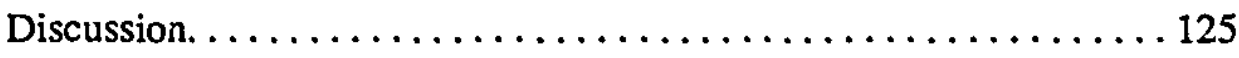

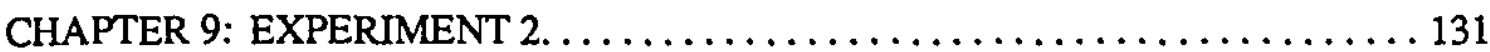

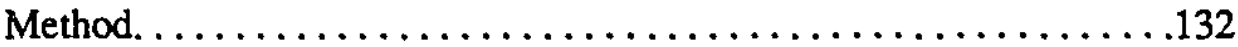

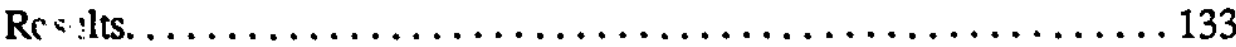

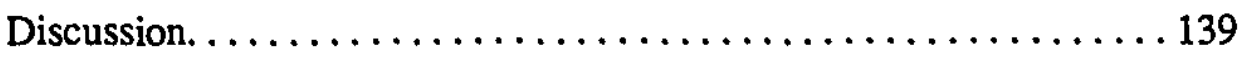

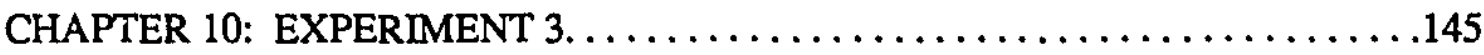

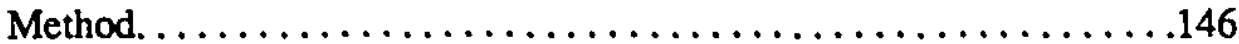

Results. ............................ 147

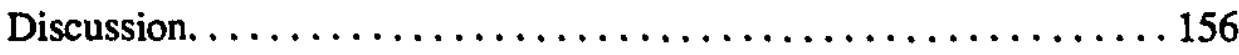

CHAPTER 11: GENERAL DISCUSSION FOR EXPERIMENTS 1, 2, AND 3. . . . 160

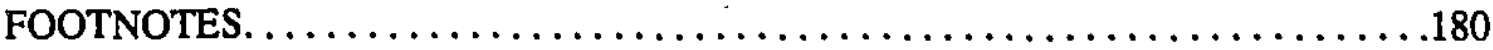

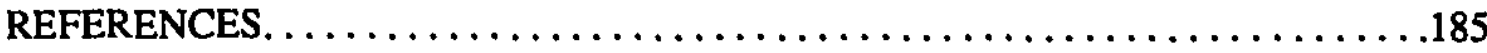

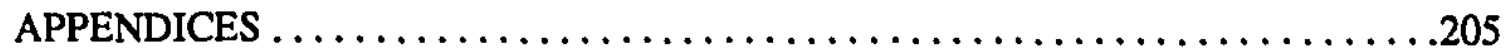




\section{Chapter 1: Introduction}

Since the mid nineteenth century and the early observations of Marc Dax and Paul Broca, it has generally been accepted that the left hemisphere is dominant for speech and language in most right-handed individuals (Joanette, Goulet \& Hannequin, 1990). The prevailing position at the time also attributed virtually no contribution of the right hemisphere to language processing. While this position has dominated until most recently, it is worth noting that some individuals at that time, including Paul Broca, attributed a more significant role to the right hemisphere in language processing (Joanette et al., 1990). Most notable among these was John Hughlings-Jackson who, based on observations that many aphasic individuals preserved intact automatic speech, proposed that "...the right is the half of the brain for the automatic use of language, the left the half for both the automatic and the voluntary use" (Hughlings-Jackson, 1915, p.82). Since these early ohservations, the importance of the right hemisphere to language processing has become increasingly recognized and more defined. While it is still maintained that the left hemisphere is dominant for language in most right-handers, it is now clear that the right hemisphere exerts an important influence on the communication process --ranging from semantic and sentence level processing skills to discourse-level phenomena.

The present set of experiments sought to more clearly define the role of the right hemisphere in language processing. In particular, the ability of right brain-damaged (RBD) individuals to use contextual information to resolve ambiguous pronouns was investigated. The motivation for the specific line of inquiry followed should become obvious in the pages to follow. Briefly, the existing literature documenting the language profile of RBD individuals has found processing deficits over a wide range of discourse level phenomena -- at the level of narratives, non-literal language, and humor. As will be elaborated upon later, a recurrent theme that has emerged from these investigations is that the nature of the communication deficit exhibited by RBD individuals relates to their difficulty in interpreting linguistic entities within the situational and discursive context of an exchange. Thus, this 
investigation attempted to methodically test this position and help to clarify the possible levels at which difficulty in the use of contextual information might exist. The investigation was extended to the left brain-damaged (LBD) population and a group of non braindamaged (NBD) controls.

Note that postulating a deficit in the use of contextual information as the underlying source for the discourse level deficits exhibited by RBD individuals, necessarily presupposes an important role for context in discourse processing. Indeed, support for the notion that context is important to discourse processing can be found when we consider its important influence in many aspects of language processing. Thus, the following review will first discuss the literature documenting the role of contextual information in normal language processing, with particular emphasis on its role in anaphor processing. This will be followed by a discussion of the use of contextual information by left brain-damaged individuals. Interestingly, while the specific issue of the use of contextual information in language processing has only recertly evolved within the RBD literature (and, as will be expanded upon later, has only really become a directly tested hypothesis in a limited number of studies), it has been the focus of an abundance of research aimed at the LBD population. Finally, the results of studies investigating the language deficits following right brain-damage will be discussed, with particular emphasis on how these results speak to the issue of a possible underlying deficit in the use of contextual information.

\subsection{Contextual influences in language processing}

\section{The influence of context on normal language processing:}

In recent years there has been considerable interest in the role that contextual information may play in normal language processing. Early investigations (e.g. Bransford \& Johnson, 1972; Dooling \& Lachman, 1971) in this area explored the effects of topics on the recall of ambiguous passages. Bransford and Johnson (1972), for example, presented 
subjects with passages such as in (1) with either no topic, a topic before, or a topic after the passage.

(1) The procedure is actually quite simple. First you arrange things into different groups depending on their makeup. Of course, one pile may be sufficient depending on how much there is to do. If you have to go somewhere else due to lack of facilities that is the next step, otherwise you are pretty well set. It is important not to overdo any particular endeavor. That is, it is better to do too few things at once than too many. In the short run this may not seem important, but complications from doing too many can easily arise. A mistake can be expensive as well. The manipulation of the appropriate mechanisms should be self-explanatory, and we need not dwell on it here. At first the whole procedure will seem complicated. Soon, however, it will become just another facet of life. It is difficult to foresee any end to the necessity for this task in the immediate future, but then one never can tell.

Topic before sentence: The paragraph you will hear will be about washing clothes.

Topicafter sentence: It may help you to know that the paragraph was about washing clothes.

Subjects were required to recall as much of the passage as possible. They found that recall was significantly better when the passage was preceded by the topic than when the topic followed the passage or when there was no topic. Bransford and Johnson (1972) interpreted these results as evidence that contextual information plays an important role in guiding language processing. The authors argued that the topic was useful in setting a context by which to interpret the subsequent passage.

Tyler and Marslen-Wilson, in a series of studies, have also found evidence to suggest that contextual information is used to guide language processing. They found that a semantic context could bias subjects' reading of syntactically ambiguous ciause fragments (Tyler \& Marslen-Wilson, 1977; Marslen-Wilson \& Young, reported in Marslen-Wilson \& Tyler, 1987). The stimuli in their study consisted of a biasing context clause ( $2 \mathrm{a}$ or b)

followed by an ambiguous word pair (3).

(2a) If you walk too near the runway...

(2b) If you've been trained as a pilot...

(3) ...landing planes...

The ambiguity of the word pair was such that it could be interpreted as either the Adjective type ("landing planes are dangerous") or the Verb type ("landing planes is dangerous"). 
The sentence fragments were presented to the subjects auditorily. Following presentation of the sentence fragments, a probe word ("is" or "are") appeared on a screen. The appropriateness of the probe word depended upon the biasing context. In the example given above, "are" would be consistent with (2a) and "is" with (2b). The subjects were instructed to name the probe word as quickly as possible. Naming latencies were recorded. The results indicated that when the probe word was inconsistent with the bias set up by the context clause, naming latency was significantly slower than when it was consistent. These results suggested that semantic context was influential in determining syntactic structure.

The results of another set of experiments by Marslen-Wilson and Tyler (1980) lend further support to the importance of context on language processing. Subjects were required to detect a target word in three types of stimulus sentences: 1) Normal Prose, in which both a syntactic and interpretative (semantic) analysis of the string was possible; 2) Syntactic Prose, which consisted of a string of words for which syntactic structuring was possible but the interpretation of the sentence was seinantically anomalous; and 3) Random Word-Order Prose which consisted of an unstructured list of words. The position of the target-words in the test-sequences was also varied in order to track the development of sources of contextual constraint across the sentence. Examples of the stimuli used are found in (4), (5), and (6) (target word is underlined):

(4) Normal Prose:

(The church was broken into last week.)

Some thieves stole most of the lead off the roof.

(5) Syntactic Prose:

(The power was located into great water.)

No buns puzzle some in the lead off the text.

(6) Random Word-Order Prose:

(Into was power water the great located.)

Some the no puzzle buns in lead text the off.

In the first experiment, a lead-in sentence (in parentheses in the above examples) was used and the results were as expected. Reacticn times were faster to target words in 
the Normal Prose than in Syntactic Prose condition, and faster in Syntactic Prose than in Random Word-Order Prose conditions. Of particular interest was the finding that targetword position had no effect in the Random Word-Order Prose condition, yet did affect reaction times in both Normal Prose and Syntactic Prose conditions. Under both conditions, there was a trend for reaction times to decrease as the target-word moved from first to ninth word position. However, the difference in reaction times to target words found under Normal Prose versus those found under Syntactic Prose remained just as great at the beginning as at the end of the sentence. It was hypothesized, therefore, that the Normal Prose condition provided an advantage over the Syntactic Prose condition due to the interpretative analysis of the sentence possible under Normal Prose. Moreover, since the first few words of an utterance are not sufficient in providing the same degree of interpretative power as would be obtained in the latter part of a sentence, the facilitation must have been provided by information contained in the lead-in sentence.

In order to check this hypothesis a second experiment was conducted that used the exact same stimuli except that the lead-in sentence was omitted. The result of this manipulation, in terms of reaction times, was to effectively eliminate the early advantage under the Normal Prose condition. These results thus provide support for the notion that discourse context is influential in the processing of sentences. Cole and Perfetti (1980) corroborated these findings using a word mispronunciation detection task. They found that reaction times to detect a mispronounced word were faster when the mispronounced word was predictable from the preceding context.

The results discussed thus far provide evidence for the influence of contextual information in language processing. However, an important theoretical issue concerns the question of at exactly what point in language processing contextual information is used. Contextual information could be used to direct initial processing decisions or instead may only be used following an initial commitment to a certain line of processing. With respect to the latter possibility, an interesting question concerns whether it is used only following 
the completion of an initial analysis or whether it is used earlier on in the analysis to guide processing operations. This question is relevant to the debate concerning the issue of whether the language processing system is modular (e.g. Altmann, 1987; Fodor, 1983; Forster, 1979; Frazier, 1987; Marslen-Tyler \& Wilson, 1987).

Advocates of modularity (Fodor, 1983; Forster, 1979) have argued that the levels of analysis involved in language processing are totally autonomous in that processing first occurs within a particular level of the language system (for example within the lexicon or syntax) and is not influenced by information higher up in the system (such as contextual information). Fodor (1983) has referred to this notion as "informational encapsulation" and has argued that a system is encapsulated in the sense that it does not have access to facts that are available to other systems. Interestingly, with respect to the influence of context on syntactic processing, however, Fodor (1983) does accept the possibility that the proposal of modularity can be maintained while acknowledging some limited interaction between the syntactic parser and what he terms the "context analyzer". He explains that it is possible that during the processing of a sentence, the parser provides the context analyzer with information concerning the particular analysis it is engaged in. The context analyzer can then use this information to determine whether or not the particular analysis is consistent with the facts provided by the context. If the analysis is consistent, the context analyzer can signal the parser to continue with the analysis. If it is not consistent, it can signal to the parser to abort that particular line of analysis. The crucial point to note is that the context analyzer cannot suggest a particular analysis to the parser, but rather can only determine whether or not a particular parse is compatible with the context. In this sense, the notion of modularity is maintained in that contextual information does not guide parsing decisions.

Opponents of the modular view of language processing (most notably MarslenWilson \& Tyler, 1987), on the other hand, argue that the levels of analysis involved in language processing are strongly interactive. In particular they propose that contextual 
information can exert a top-down influence on language processing and in this way direct the initial analysis. With respect to the studies just described (i.e. Tyler \& Marslen-Wilson, 1977; Marslen-Wilson \& Tyler, 1980), they have argued (Marslen-Wilson \& Tyler, 1987) that because of the early context effects demonstrated and the apparent speed of processing, the empirical distinction between a modular model and an interactive model is difficult to make.

Investigations into the effect of contextual information on lexical and syntactic ambiguity resolution have attempted to distinguish the locus of effects. Not surprisingly, the results have been mixed. With respect to lexical ambiguity resolution, until recently, the evidence provided by a number of studies (for example, Foss \& Jenkins, 1973; Swinney, 1979; Tanenhaus, Leiman \& Seidenberg, 1979) using a variety of methodologies, supported the "post decision hypothesis" (Swinney, 1979). This hypothesis maintains that all meanings of an ambiguous word are initially accessed and contextual information is used only to choose the appropriate meaning following initial lexical access.

More recently, however, the notion that contextual information is only used following lexical access has been challenged (Paul, Kellas, Martin \& Clark, 1992) and there is some suggestion that context effects may be modulated by lexical factors. For example, Tabossi, Colombo and Job (1987) and Tabossi (1988) have demonstrated contextual facilitation effects for the dominant, but not subordinate, meanings of ambiguous words. In a cross-modal lexical decision task it was found that if a sentential context for the dominant meaning of an ambiguous word was "sufficiently constraining" (i.e. it highlighted a particularly remarkable characteristic of the dominant meaning) then reaction times to a target word related to the dominant meaning were faster than reaction times to a target word that was either related to the subordinate meaning, or was unrelated to either meaning (with no significant difference in reaction times to the latter two). If, on the other hand, the context biased the subordinate meaning, both meanings of the 
ambiguous word were accessed, reflected in a lack of difference in reaction times to targets related to either meaning of the word. Thus, it was concluded that contextual information can influence lexical access, under certain circumstances. MacDonald, Pearlmutter and Seidenberg (1993) have recently reported similar evidence to suggest that context effects may be modulated by the dominance (or frequency) of meaning. They also found contextual facilitation effects for meanings of ambiguous words that were of high frequency, but not for those of low frequency.

The studies investigating the locus of context effects in syntactic ambiguity resolution have been equally inconclusive. Two primary positions have been advanced. One position maintains that the initial parsing of a sentence is completely autonomous and, therefore, uninfluenced by other factors such as contextual information. The most well debated theory reflecting this position is that of the "garden-path" theory advanced by Frazier and her colleagues (Frazier, 1978; 1987; Frazier \& Rayner, 1982; Rayner, Carlson, \& Frazier, 1983). According to the garden-path theory of sentence processing, the parser initially commits itself to only one parse and the initial commitment to an analysis is totally uninfluenced by other considerations, including the use of contextual information. The theory maintains that contextual information is only used following the initial parse, to help recover from misanalyses.

In contrast to this position, Altmann and Steedman (1988; Altmann, 1987, 1989; Steedman \& Altmann, 1989) have proposed a significantly more influential role for contextual information in early sentence processing. They have advanced the "Incremental Interactive" theory of sentence processing which views the "human sentence processor" as having a "parallel, fine-grained, weakly interactive architecture" (Altmann \& Steedman, 1988, p. 205). They have argued that the processor is not strongly interactive in that it does not specifically direct syntactic processing by proposing a certain course of analysis. Instead, they view the sentence processor as weakly interactive in that it evaluates analyses that have been automatically generated against semantic and contextual information. The 
important point to note is that the processor is not predisposed to a particular analysis based on contextual information. Note that this view is compatible with the notion of modularity advanced by Fodor (1983) in which he accepts the possibility of a "context analyzer". In terms of the sentence processor being described as fine-grained, it is argued that interactive processing and, hence, the use of contextual information, can occur on very small syntactic units (perhaps as small as the word). Finally, the human sentence processor is described as parallel, thereby implying that alternate analyses of a sentence are offered in parallel, not serially (as proposed by Frazier's (1978) garden-path theory).

It is important to note that both the garden-path theory and the incremental interactive theory of sentence processing adhere to the notion of modularity within the language system. The key difference between the two positions concerns the point at which contextual information comes into play. The garden path theory posits a role for contextual information only following a completed analysis, to aid in the recovery of the interpretation. In contrast, the incremental interactive theory views contextual information as being influential during the analysis -- not to direct it initially, but to guide it before the analysis is completed. Experimental evidence supporting both positions has been found in a number of studies examining the effects of manipulating both intrasentential semantic and pragmatic information (Ferreira \& Clifton, 1986; Rayner et al., 1983; Trueswell, Tanenhaus, \& Garnsey, 1993) and previous discourse on the resolution of syntactic ambiguities (Altmann, 1987; 1989; Altmann, Gamham \& Dennis, 1992; Altmann \& Steedman, 1988; Crain, 1980 reported in Crain \& Steedman, 1985; Clifton \& Ferreira, 1989; Ferreira \& Clifton, 1986; Mitchell, Corely \& Garnham, 1992; Murray \& Liversedge, 1993; Rayner, Garrod \& Perfetti, 1992; Steedman \& Altmann, 1989).

To summarize, a considerable amount of interest within the psycholinguistic literature has concerned the influence of contextual information on language processing. The evidence supports an important role for context in language comprehension; however, the evidence does not so clearly specify the point at which contextual information is 
influential in processing. The question remains as to whether it is used early to guide parsing decisions (Altmann, 1987; 1989; Altmann \& Steedman, 1988; Steedman \& Altmann, 1989) and possibly even to direct them (Marslen-Wilson \& Tyler, 1980; 1987; Tyler \& Marslen-Wilson, 1977) or during an off-line process following an initial parse (Clifton \& Ferreira, 1987; 1989; Ferreira \& Clifton, 1986; Frazier, 1978; 1987; Frazier \& Rayner, 1982; Rayner et al., 1983).

\section{The influence of context on anaphor resolution:}

Anaphora may be defined as, "... the relation between a 'proform' (called an 'anaphor') and another term (called an 'antecedent'), wherein the interpretation of the anaphor is in some way determined by the interpretation of the antecedent" (Lust, 1986, p.9). Types of anaphoric forms include lexical replacement (e.g. The Buick is on the street. That car still looks brand new), repeated forms (e.g. Tom went to the store. Tom bought some bread), pronominal forms (e.g. Sally is in the school play and she is a princess), and verb-phrase ellipsis (e.g. Someone had to paint the room so Tom did __), among others. The resolution of all types of anaphors requires the listener or reader to replace the form with a representation of the antecedent to which it refers.

Anaphora is important to discourse processing in that it is viewed as an important linguistic device for achieving cohesion. Cohesion, as defined by Halliday and Hasan (1976), "... refers to the relations of meaning that exist within the text and that define it as a text" (p.4). These semantic relations are necessary for the interpretation of the text (or discourse). Given its paramount role in establishing successful discourse, many researchers have sought to identify the factors involved in anaphor resolution. Not surprisingly, a large amount of research has focused on the influence of discursive factors (i.e. those related to the use of discourse and contextual information) on the resolution of anaphors. Emerging evidence suggests that while specifically linguistic factors (i.e. those related to syntax and semantics) help to constrain and direct anaphor resolution, final interpretation is often subject to contextual considerations. Before discussing the evidence 
related to the influence of discursive factors on anaphor resolution, a brief review of specifically linguistic factors is in order.

With respect to linguistic factors, some general lexical/syntactic properties have been identified as aiding in the resolution of certain types of anaphors. For example, in the case of personal pronouns, knowledge of gender and number is often sufficient for determining the correct antecedent. On a more heuristic level, in the absence of other sources of information, the noun phrase in the subject position of a sentence is often the preferred referential candidate for an anaphor in the following sentence or clause. In fact, this strategy of choosing the subject of the sentence as the referent results in correct resolution approximately $90 \%$ of the time in written texts and $75 \%$ of the time in dialogues (Hobbs, 1979). Along a similar vein, Sheldon (1974) has proposed the use of a "parallel function strategy" in resolving pronominal reference which states that in the case of a conjoined sentence, "...the pronoun in the second conjunct is interpreted as being coreferential with the NP that has the parallel grammatical function in the first conjunct" (p.280). Of course, more formal generative accounts have also been offered (e.g. Langacker, 1969; Lasnik, 1976; Reinhart, 1983) which have postulated structural conditions which permit and constrain pronominal reference. In addition to these lexical/syntactic factors, a semantic factor, termed implicit causality, has also been identified as influencing pronoun resolution. Given that this factor is directly relevant to the series of experiments which comprise this investigation, it will be discussed in detail.

The notion of implicit causality relates to the fact that the very nature of some verbs conveys attribution for the particular action or emotion stated by the verb. In a series of sentence completion studies, Garvey and his colleagues (Garvey \& Caramazza, 1974; Garvey, Caramazza \& Yates, 1976) found that when subjects were asked to complete a sentence fragment of the form, NPI V(ed) NP2 because Pronoun, the assignment of the pronoun to either the first or second noun-phrase was largely determined by the verb used. For example, the verb telephoned as in, John telephoned Bill because he..., consistently 
induced such completions as, ...had good news, which clearly identified the cause of the action, to telephone, to the first NP, "John". On the other hand, with a verb such as admired as in, John admired Bill because he..., completions such as, ...was a fine athlete, were consistently induced. In this case the cause of the emotion was clearly designated to the second NP, "Bill". Thus, each verb tested was identified as having either an NP1 bias or an NP2 bias. It should be noted that the bias implicit in the verb which assigns causality to either the first or second noun-phrase was not found to be complete for all verbs studied. Rather, the results indicated that the strength of the bias for any given verb should be viewed as being along a continuum.

The strong influence of the implicit causality of a verb on the assignment of referents for ambiguous pronouns was further demonstrated by Caramazza, Grober, Garvey and Yates (1977) using a timed comprehension task. Pairs of sentences of the form Cynthia sold the bike to Maureen because she needed cash/could pay cash were presented visually to a group of subjects. Their task was to decide to whom the pronoun referred in each sentence, by vocalizing the name of the referent. The stimuli were constructed such that for each verb, the interpretation produced by the reason provided in the subordinate clause for the action or emotion stated by the verb in the first clause, was either consistent or inconsistent with the verb's bias. It was hypothesized that if the implicit causality of a verb was an important factor in determining reference, then vocalization latencies should be longer when the interpretation of the sentence was inconsistent, rather than consistent, with the verb's natural bias. The results supported this hypothesis. Vocalization latencies to the preferred referent were shorter when the interpretation that was produced was consistent with the verb's natural bias than when it was inconsistent with it. As pointed out by Vonk (1984; 1985b), the exact linguistic basis for this phenomenon is unresolved (cf. Au, 1986; Brown \& Fish, 1983; Fiedler \& Semin, 1988). However, the effect of implicit causality on pronoun resolution appears to be quite robust (cf. Ehrlich, 1980) and has been confirmed by a number of studies (e.g. Garnham, 
Oakhill \& Cruttenden, 1992; Grober, Beardsley, \& Caramazza, 1978; Light and Capps, 1983, cited in Light and Albertson, 1988; Vonk, 1985a; 1985b).

Returning to the notion that discursive factors (relating to both linguistic and situational contexts) influence anaphor resolution, it might be noted that, at first glance, this proposal seems trivial. Obviously context must influence anaphor resolution in that it is in the context that the antecedent to an anaphor must be found. However, it is important to realize that this is not what is at issue when discussing contextual effects on anaphor resolution. Rather, the interesting question concerns how or if context can influence the decision for a preferred antecedent, especially in the case of ambiguous reference.

Bosch (1983) and Brown and Yule (1983) have addressed the issue of the importance of context to anaphor resolution in terms of "context models" and "discourse representations", respectively. Both propose essentially the same notion. In particular, both view the participants of a discourse as continually developing models, as the discourse evolves, that serve to represent the environment in focus. The development of these models is a function of the participants' background knowledge. Background knowledige includes general world knowledge, sociocultural knowledge, stereotypes, the awareness of a shared context, and interpretation of previous utterances. Speakers, therefore, when using an anaphor, will do so upon considering the hearer's discourse model and will choose an intended referent that is believed to be within the hearer's model. Similarly, it is believed that hearers will also be operating with this strategy in mind, and resolve the anaphor based on their present discourse model.

Experimental evidence for the influence of discourse structure has been found. For instance, it has been demonstrated that the notion of "focus", identified by Grosz and her colleagues (Grosz \& Sidner, 1986; Grosz, Pollack \& Sidner, 1989) as relating to the "attentional state" of the discourse participants, is a factor in constraining antecedent choices. Focused (or centered) elements appear to be the preferred referents for anaphors. For instance, Hudson, Tanenhaus and Dell (1986, reported in Sanford \& Garrod, 1989) 
presented subjects with a context-setting sentence like (7) followed by a pronominal sentence as in (7a) or (7b). The referent of the pronoun was either the agent of the verb in the context-setting sentence (as in 7a) or the patient (as in 7b).

(7) Jack apologized profusely to Josh.

(7a) He had been rude to Josh yesterday.

(7b) He had been offerided by Jack's comment.

According to Hudsoni et al., the agent of the verb ("Jack" in the example above) is the centered or more focused element of the sentence, and therefore should be the preferred referent for the pronoun. The results were coissistent with this hypothesis. Reading times were significantly faster to sentences like (7a), in which the correct referent was the centered target, than to sentences like (7b), in which the correct referent was the noncentered target (i.e. the patient of the verb).

Garrod and Sanford (1985, Experiment 2) found similar evidence to support the notion that elements within a discourse focus have preferential status as referents for pronouns. They used a spelling detection task to investigate the immediacy of interpretation of anaphoric expressions in discourse. The first experiment was concerned with the interpretation of proper nouns and definite descriptions. Subjects were presented with a text that included a "scene-setting" sentence which served to introduce the main character (realized as a proper noun) and the secondary character (realized as a definite description). The target sentences were constructed such that the anaphor was in the subject position, and the verb was consistent with one character but not the other. An example of the stimulus set is provided in (8).

(8) Title: A dangerous incident at the pool.

Context: Elizabeth was a very inexperienced swimmer and wouldn't have gone into the pool if the lifeguard hadn't been nearby. But as soon as she was out of her depth she started to panic and wave her hands about in a frenzy. 
Target sentences:

(a) Within seconds Elizabeth jumped into the pool.

(b) Within seconds the lifeguard jumped into the pool.

(c) Within seconds Elizabeth sank beneath the surface

(d) Within seconds the lifeguard sank beneath the surface.

Follow on: She was lucky that he had been attending.

Misspellings: jumped - jimped; sank - senk

The authors reasoned that in order to determine the consistency of the verb to the subject, resolution of the anaphoric expression with respect to the preceding discourse must first be completed. For example, in the case presented in (8), the inconsistency of the verb "jumped" with respect to the subject "Elizabeth" can only be determined with reference to the context, which indicates that Elizabeth is in the pool, and to world knowledge which indicates that it is improbable for someone to "jump" when they are floating in a pool. Subjects were required to indicate detections of spelling errors as quickly as possible. It was hypothesized that if the anaphor was being resolved immediately with respect to the preceding context, detection of verb spelling errors (as a measure of the ease with which a word is recognized) should be faster when the verb was consistent with the subject of the sentence (i.e. the anaphor) than when it is inconsistent with it. The results were in support of this hypothesis and indicated that spelling error detection was significantly faster for consistent than for inconsistent verbs.

A second experiment was conducted which investigated the immediacy with which anaphoric pronouns were interpreted. Materials similar to those described above were used except that a pronoun served as the anaphoric expression. The results were particularly interesting with respect to their implication for the influence of discourse focus on pronominal reference. It was found that the anaphor-verb consistency effect found in Experiment 1 held only for the case where the pronoun referred to the main character of the story. The authors interpreted this result in terms of focusing within the discourse model. They pointed out that in this experiment, since the secondary characters wr.e not the thematic subjects of the discourse, they did not warrant the special status of being in focus 
when a referent search was activated. Thus, a pronoun referring to a secondary character was not immediately integrated with the contextual information provided by the discourse, resulting in no effect of verb consistency. The authors concluded, therefore, that the immediacy of use of contextual information is dependent upon the elements in focus. Furthermore, they suggested that different anaphoric expressions have different requirements for the limits of focused information such that pronouns which appear to serve a maintenance function and are clearly under-specified, require a much more limited focus system than the more fully specified anaphoric expressions such as proper nouns. Overall, therefore, the results of Garrod and Sanford (1985) suggest that contextual information can serve to constrain the pragmatic choices for antecedents, but that the immediacy of use of context in resolving pronouns is largely a function of the elements in focus.

The use of contextual information to constrain pragmatic choices for antecedents has also been found by Hirst and Brill (1980). They investigated subjects' abilities to choose the referent of an ambiguous pronoun, when the preferred choice was largely determined by the plausibility of the situation detailed in the sentences. They presented subjects with leading sentences such as (9). Once the leading sentence was read it disappeared from view and a sentence beginning with the pronoun "he" as in (9a) and (9b) appeared. Subjects were required to choose the correct referent of the pronoun "he" by pressing a response key corresponding to either "John" or "Henry".

(9) John stood watching while Henry fell down some stairs.

(9a) He ran firr a doctor.

(9b) He thought of the future.

The pragmatic plausibility of the information contained in the second sentence with respect to the information contained in the leading sentence was manipulated. In some cases, the pronominal sentence largely favored one of the two antecedents based on praginatic reasoning. In this example, for instance, the preferred referent of "he" in (9a) is clearly 
"John" as it is more plausible that someone "watching", and not "falling", is in a better position to "run for a doctor". In other cases, there was no preferred referent as in (9b), where it is equally likely for both referents to "think about the future". It was hypothesized that if contextual information was influential in pronoun assignment, then referent selection by the subjects should be a function of the plausibility of the sentences. The results were in support of this hypothesis. Reaction times to choose the preferred referent were significantly faster to sentences which highly favored a particular referent based on pragmatic plausibility such as in (9a), than to those that were more ambiguous, as in (9b). Light and Capps (1986) reported similar results.

In line with the findings of Hirst and Brill (1980), Tyler and Marslen-Wilson (1982; see also Marslen-Wilson, Tyler \& Koster, reported in Marslen-Wilson \& Tyler, 1987) also found evidence to suggest that pragmatic inference based on the use of contextual information plays a role in anaphor resolution. They presented subjects with stimulus items consisting of a context such as (10) followed by a continuation fragment $(10 \mathrm{a}, \mathrm{b}$, or $\mathrm{c}$,$) .$

(10) As Philip was walking back from the shop, he saw an old woman trip and fall flat on her face. She seemed unable to get up again.

(10a) Philip ran towards...

(10b) He ran towards...

(10c) Running towards...

The sequences of sentences were presented auditorily to the subjects. Following the presentation of the incomplete continuation fragment, a probe word was presented visually and subjects were required to name it. The probe words "him" and "her" were used for the examples given above, with "him" being the inappropriate probe in all cases. Longer naming latencies to inappropriate probes were expected. It is important to note that any difference in naming latencies between appropriate and inappropriate probes required first 
that the anaphor (the agent of the verb) was resolved. Otherwise, both "him" and "her" would be equally appropriate.

The key difference between (10a,b,or c) hinged on the type of anaphoric device used. In (10a), the device used was a repeated noun phrase. In (10b), an unambiguous personal pronoun was used. In (10c), however, no explicit linguistic cues were used. Correct resolution depended on pragmatic inference (i.e. determining the most likely candidate to be running). The results indicated equally significant differences between appropriate and inappropriate continuations in all three conditions and, thus, provided support for the notion that pragmatic inference based on contextual information is used in anaphor resolution.

Of particular interest to the issue of the use of contextual information in resolving anaphors has been the mounting evidence to suggest that contextual information may even be used when the choice of the antecedent may be constrained purely on linguistic grounds. Hirst and Brill (1980), for instance, who, as just reported, found evidence for the use of contextual information to constrain pragmatic choices of antecedents, conducted a second experiment in which the referent was constrained on purely syntactic grounds. They transformed the sentences used in Experiment 1 such that the referent of the pronoun could be unambiguously determined, while preserving the pragmatic plausibility of the sentences, as in (11).

(11) John stood watching. He ran for a doctor after Henry fell down some stairs.

Once again, subjects were presented with a leading sentence followed by a pronominal sentence and required to explicitly specify the referent of "he". The results indicated that the contextual information still had an effect, reflected in faster reaction times to sentences with stronger plausibility constraints, as in (11) compared to those with weaker plausibility constraints as in, "John stood watching. He laughed with a vengeance after Henry fell down some stairs". 
Similar results were found by Stevenson and Vitkovitch (1986, Experiment 2) using materials adapted from Hirst and Brill (1980). In addition to manipulating both the pragmatic plausibility of the sentences and syntactic constraints (pronominal reference vs. ellipsis), they manipulated gender cues such that half of the sentences were unambiguous based on gender. Examples of the type of stimuli used are provided in (12) and (13).

(12) Jane stood watching. Henry jumped across a ravine and (he) fell into the river. (High plausibility)

(13) Jane stood watching. Henry jumped across a ravine and (he) picked up some money. (Low plausibility)

The task was the same as that used in Hirst and Brill (1980). Consistent with the findings of Hirst and Brill (1980), reaction times to assign the referent to sentences of low plausibility were significantly slower than to those of high plausibility, even when the assignment was unambiguous based on gender cues. The authors interpreted this result as evidence that assignment of a referent is delayed until contextual information following the pronoun is integrated into the discourse model. This view was also supported by Experiments 1 and 3 of Stevenson and Vitkovitch (1986). Both experiments compared anaphor resolution in pronoun and elliptical sentences under two verb conditions -- verb informative, as in (14) and verb uninformative, as in (15).

(14) Jane was late for her appointment with Sue and (she) hurried to get a taxi.

(15) Anna lent Felicity the steam iron and (she) forgot to give the instructions.

In the informative verb condition, the information contained in the verb was sufficient to disambiguate the referent based on general world knowledge (for example, a person who is late is likely to be the one hurrying). In the uninformative verb condition, the verb was neutral with respect to the conditions set up in the first clause and it was only by reading the information following the verb that the preferred referent could be identified. Notice, however, that in the elliptical sentences, the anaphor is also constrained by the syntax. 
Consequently, if assignment is immediate, there should be no difference in responses between the informative and uninformative verb conditions for elliptical sentences. On the other hand, if assignment is delayed, faster reaction times should be found to sentences in the informative verb condition compared to those in the uninformative verb condition for both pronoun and elliptical sentences. Indeed, it was found that even though, in the case of elliptical sentences, assignment was constrained by the syntax, reaction times to determine who was performing the action in the second clause (in Experiment 1) and reading times (in Experiment 3) were longer for sentences in the uninformative verb condition.

Interestingly, the results from some of the studies on the effect of implicit causality on pronoun resolution may also be interpreted as support for the integration of contextual information following the appearance of a pronoun. The information provided in the second clause of the "because" sentences used in these studies (e.g. Cynthia sold the bike to Maureen because she needed cash/could pay cash) must be integrated with the information in the first clause in order to both react to the bias of the verb (in terms of its consistency or inconsistency) and to find the correct referent.

Finally, it should be noted that it is important to distinguish between initial coindexation of a pronoun and final assignment. The results of Hirst and Brill (1980) and Stevenson and Vitkovitch (1986) seem to suggest that assignment is delayed until information subsequent to the anaphor is processed. In this sense, contextual information following the pronoun is useful in the resolution of the pronoun in terms of integrating the interpretation of the pronoun within a discourse model. However, whether or not coindexation of the anaphor is initiated immediately upon encountering it is not specifically addressed by their results nor can this possibility be discounted. In fact, there is some evidence to suggest that, at the very least, the referent is activated very early upon encountering the anaphor (Dell, McKoon, \& Ratcliff, 1983; Stevenson, 1986, reported in Sanford \& Garrod, 1989; Tyler \& Marslen-Wilson, 1982). Furthermore, due to the offline nature of the tasks using explicit assignment, it is possible that assignment is actually 
completed, if possible, before the end of the sentence, based, for instance, on syntactic considerations. According to this view, contextual information following the pronoun may, therefore, have only influenced the actual response, resulting in faster reaction times to referents that are contexiually supported, simply because "two cues are better than one" (as suggested by Garrod \& Sanford (1985) concerning other results).

To summarize, it appears that a number of factors are involved in determining the influence of contextual information on pronoun resolution. Certainly, preceding discourse structure may be involved in constraining antecedent choices while information subsequent to the anaphor may be involved in its full integration into the discourse. The question of immediacy of contextual integration of an anaphor remains. Emerging evidence suggests that resolution may be initiated immediately with the use of certain cues such as gender cues, implicit causality, and discourse focus, but that the process may not be completed until information following the pronoun has been processed (Sanford \& Garrod, 1989; Vonk, 1985b).

The preceding discussion has focused on the influence of contextual information on normal language processing -- ranging from the resolution of lexical and syntactic ambiguities to anaphor resolution. Interestingly, further support for the importance of contextual information on language processing can be found in the literature on LBD individuals.

\subsection{Contextual influences on language processing by LBD individuals}

It is well documented that significant linguistic deficits frequently emerge as a function of left brain-damage. Interestingly, however, the functional communicative competence of LBD individuals in everyday discourse is often much better than would be predicted based on their linguistic abilities. Left brain-damaged patients have been shown to be capable of using contextual information involving both linguistic and extralinguistic cues, as well as evaluations based on general word knowledge and semantic plausibility. 
Thus, one hypothesis that has been advanced to account for the apparent discrepancy frequently observed between the linguistic and functional communicative abilities of these patients is that contextual information plays an important role in their communicative competence.

Several studies have examined the influence of extralinguistic cues on the language comprehension abilities of LBD patients. For example, Wilcox, Davis \& Leonard (1978) have demonstrated that the processing of indirect speech acts by aphasic individuals is considerably aided when presented in naturalistic settings as opposed to in isolation as in standard test batteries. Pictures have also been found to aid in the interpretation of utterances by low comprehending aphasics by Pierce and Beekman (1985). In contrast, however, other studies (e.g. Hough, 1990; Waller \& Darley, 1978) have not found pictorial contexts to be beneficial in the processing of text by aphasic individuals. However, methodological factors such as the length of the experimental stimuli have been advanced to account for the discrepant results (Hough, 1990).

Studies investigating the use of linguistic contextual information by LBD patients have assessed the use of contextual information present within the sentence as well as that contained outside the sentence boundary. With respect to contextual information contained within the isolated sentence, it has been frequently demonstrated that comprehension of sentences by aphasic individuals is better when the processing of the sentence is constrained by the semantic plausibility of the events depicted (Caramazza \& Zurif, 1976; Deloche \& Seron, 1981; Heeschen, 1980; Kudo, 1984). For example, Caramazza and Zurif (1976), using a sentence picture matching task, found that the interpretation of object relative center-embedded sentences by aphasic individuals was significantly worse for reversible (e.g. The boy that the girl is chasing is tall) and improbable (e.g. The boy that the dog is patting is fat) sentences than for semantically constrained nonreversible sentences (e.g. The apple that the boy is eating is red). As well, comprehension of target words by aphasic subjects has been found to be facilitated by the inclusion of semantically related 
words within the sentences (e.g. "You see a cat that is furry" versus "You see a cat that is nice", target = cat $)($ Gardner, Albert \& Weintraub, 1975; Clark \& Flowers, 1987). Similarly, Pierce (1982) has demonstrated that the processing of tense and word-order by aphasics is aided by the existence of redundant markers (e.g. "already" to signify past tense).

Concerning the use of information contained outside the sentence boundary, converging evidence from a number of studies suggests that the interpretation of sentences and paragraphs by aphasics is influenced by preceding linguistic contexts (Cannito, Jarecki and Pierce, 1986; Friederici, 1983; Germani \& Pierce, 1992; Hough, Pierce \& Cannito, 1989; Pierce, 1988; Pierce \& Beekman, 1985; Pience \& DeStefano, 1987; Pience \& Wagner, 1985; Waller and Darley, 1978). Waller and Darley (Experiment 2, 1978), for instance, found that aphasics' comprehension of paragraphs was significantly improved when preceded by a short context of two or three sentences that stated information concerning what was about to be described. In contrast, however, Waller and Darley (1979) failed to find an effect of context for aphasic individuals in the processing of various syntactically complex sentences that have been shown to pose problems for them (e.g. passive sentences, sentences with "before", "after", "under"). A possible explanation to account for these discrepant findings is provided by the results of Pierce and his colleagues. In a series of studies (Cannito et al., 1986; Germani \& Pierce, 1992; Hough et al., 1989; Pierce \& Beekman, 1985; Pierce \& DeStefano, 1987; Pierce \& Wagner, 1985), they have found evidence to suggest that the discrepant results may be accounted for by the severity of the aphasic patients' comprehension deficits. In particular they found that only aphasics with low comprehension skills benefited from the presence of a preceding linguistic context (Cannito et al., 1986; Pierce \& Beekman, 1985; Pierce \& Wagner, 1985). As discussed by Pierce and Wagner (1985), such a distinction may help to account for the discrepant findings of Waller and Darley (1979) since the aphasics used by Waller and Darley (1979) were quite high functioning. In addition to the possibility that the level of 
auditory comprehension affected the findings in the different studies, Pierce and Wagner (1985) also noted that the contexts used in the Waller and Darley (1979) study were more general in nature, whereas in Pierce and Beekman (1985) and Pierce and Wagner (1985) they clearly biased a particular interpretation.

Another interesting finding derived from this series of studies relates to whether or not the preceding context is only beneficial to aphasic individuals when it is predictive of the ensuing action. The study by Pierce and Wagner (1985), in which the preceding context consisted of only one sentence suggested that contextual information was only useful when it clearly biased a particular interpretation. However, more recent studies (Cannito et al., 1986; Germani \& Pierce, 1992; Hough et al., 1989) have found evidence to the contrary. In these studies, the preceding context consisted of short narratives and it was found that the interpretation of target sentences by aphasics was facilitated by paragraphs that were both predictive and nonpredictive. The authors have argued that the advantage in processing the target sentences gleaned from information contained in the nonpredictive paragraphs is based on redundancy of information. Hough et al. (1989) have proposed that both predictive and nonpredictive contexts provide relevant information that allow the listener or reader to familiarize themselves with the characters and actions involved, to such an extent that when the target sentence is processed, most of the subjects' attentional resources can be directed towards determining the syntactic relationship among the participants. However, when a sentence is presented in isolation or preceded by only a single nonpredictive sentence (as in Pierce \& Wagner, 1985), processing resources must be directed to not only determining the syntactic relationship among participants, but to determining who and what is/are involved.

The notion that redundancy of information aids aphasic individuals in processing discourse was also advanced by Stachowiak, Huber, Poeck, and Kerschensteiner (1977). They investigated subjects' abilities to choose a picture that best depicted the interpretation of an idiom presented in a narrative. Contrary to expectations, they found no difference 
between normals and aphasics in their abilities to correctly choose the target picture. They concluded that the redundancy of information presented in the narratives facilitated comprehension for the aphasics. Interestingly, Kohlert (1979, reported in Huber, 1990) qualified this interpretation. He used the same stimuli as in Stachowiak et al. (1977) but reduced the amount of informational content in the stories. As well, one less foil was used in the picture stimuli. The effect of these changes was that the number of errors made by normals was reduced, but those made by aphasics remained high. According to Huber (1990), therefore, it seems that redundancy only had an effect in that the narratives became longer and more complicated and thereby induced more errors in normals -- thereby lessening the gap between the scores for normals and aphasics.

An important finding in the literature regarding the abilities of LBD individuals to process discourse has been the consistent demonstration that their comprehension of discourse cannot be predicted from tests aimed at assessing sentence comprehension. Numerous studies (Stachowiak et al., 1977; Waller \& Darley, 1978; Wegner, Brookshire \& Nicholas, 1984) have found a dissociation between sentence comprehension skills and discourse comprehension such that discourse comprehension may be relatively intact although accompanying syntactic comprehension deficits exist. Caplan and Evans (1990) specifically manipulated syntactic complexity at both the sentence level (using only irreversible sentences) and at the discourse level. 'They were interested in the possibility that LBD patients may respond differentially to stories comprised of complex syntactic constructions versus those with more simple syntactic constructions. They hypothesized that if aphasics have syntactic comprehension difficulties, comprehension of complex stories should be reduced relative to comprehension of simple stories. Interestingly, the results did not support this hypothesis. While LBD individuals evidenced syntactic complexity effects at the level of the isolated sentence, they did not show any differences between the processing of syntactically simple or complex stories. These results provided strong support for the view that aphasic individuals may demonstrate sentence 
comprehension difficulties, yet still be capable of understanding language at the discourse level.

Attempts to explain how aphasics are capable of understanding discourse irrespective of sentence comprehension deficits have led many researchers to propose that they retain the ability to extract the macrostructure of a discourse (which is based on more heuristic processing related to general world knowledge) and, therefore, do not need to engage in microprocessing (which is necessarily more specifically linguistic) (Huber, 1990). Evidence for the use of macroprocessing of narratives by aphasics is found in a study by Huber and Gleber (1982). They investigated aphasics' abilities to order scrambled sentences and pictures into coherent narratives. In the sentence arrangement task they manipulated the cohesiveness (a microprocess) of the narratives such that there were both low and high cohesion versions. They reasoned that if the patients were processing the narratives based predominantly on their macrostructures then there should be no difference between performance on the low and high cohesion versions. The results supported their hypothesis. No significant differences were found between the processing of low and high cohesion versions.

The notion that aphasics retain knowledge of narrative macrostructure has also been supported in a series of production tasks by Ulatowska and her colleagues (Ulatowska, Allard, \& Bond-Chapman, 1990; Ulatowska \& Bond, 1983; Ulatowska, Doyel, Freedman-Stern, Macaluso-Ȟiaynes, \& North, 1983; Ulatowska, Freedman-Stern, WeissDoyel, Macaluso-Haynes, \& North, 1983; Ulatowska, North, \& Macaluso-Haynes, 1981). These studies have consistently found that mildly and moderately, but not severely impaired aphasics have preserved knowledge of narrative structure as evidenced by their use of essential "superstructure" elements (settings, complications and resolutions) in their narrative accounts. These findings have been corroborated by Glosser and Deser (1990) using similar narrative tasks. Differences that emerged between the narrative and procedural discourse produced by aphasics and normals were more quantitative rather than 
qualitative in nature. Aphasics generally produced less discourse and the complexity of the language used was reduced.

To summarize, there is consistent evidence to suggest that aphasic individuals use contextual information to process language. Factors that appear to modulate the degree of influence of contextual information include the level of auditory comprehension exhibited by the patient (low comprehenders appearing to benefit from its use but not high comprehenders) and the amount of redundancy of information (Cannito et al., 1986; Germani \& Pierce, 1992; Hough et al., 1989; Pierce \& Beekman, 1985; Pierce \& Wagner, 1985). It appears that redundancy in a context is important for aphasic individuals in establishing a contextual model upon which the interpretation of utterances may be based. Important as well has been the demonstration that, irrespective of sentence level comprehension deficits, aphasic individuals may still be capable of engaging in macroprocessing and thereby satisfactorily comprehend (Caplan \& Evans, 1990; Stachowiak et al., 1977; Waller \& Darley, 1978; Wegner et al, 1984) and produce language at the discourse level (Glosser \& Deser, 1990; Ulatowska et al., 1990; Ulatowska \& Bond, 1983; Ulatowska et al., 1983; Ulatowska et al., 1983; Ulatowska et al., 1981), consistent with clinical observations.

It is interesting to note, however, that while LBD individuals have been shown to be influenced by contextual information in many aspects of language processing, there is some suggestion that they may have difficulty in using linguistic contextual information to resolve pronouns. Evidence for this was suggested by the results of Grober and Kellar (1981). They investigated the sensitivity of aphasic individuals to the implicit causality of verbs. As in Caramazza et al. (1977), they presented subjects with sentences of the form NP1 Verbed NP2 because Pronoun Reason (e.g. John telephoned Bill because he needed some information). The information provided in the subordinate clause was either consistent or inconsistent with the bias of the verb. Only aphasic patients were tested and they were required to answer questions which queried the referent of the pronoun in the 
target sentences (e.g. "Who needed some information?"). The results indicated an effect of implicit causality. More errors were made on inconsistent than consistent sentences. Moreover, this result held even when the pronoun could be disambiguated based on gender cues alone (e.g. John telephoned Sue because he needed some information); nevertheless, fewer errors were made overall when the pronoun was unambiguous due to gender constraints. These results paralleled the reaction time data of Caramazza et al. (1977) and provided evidence that the semantic property of verbs termed implicit causality is preserved in aphasic patients. Interestingly, however, the results also suggested that aphasics have difficulty integrating the information between both clauses to resolve the pronoun. As has been pointed out previously, in order to successfully resolve the pronoun in inconsistent sentences the information in the second clause must be integrated with that of the first clause. In this study the aphasic patients demonstrated considerable difficulty resolving ambiguous pronouns for inconsistent sentences, responding close to chance (mean percent accuracy $=53$ ).

Difficulty in using linguistic context to resolve pronouns has also been suggested by the results of a study by Chapman and Ulatowska (1989). They presented subjects with short stories (four sentences long). The leading sentence of each story introduced two characters of the same gender. Subsequent sentences referred to these characters either through the use of a repeated noun phrase anaphor (repetition of the name) or a pronoun. The plausibility of events involving the characters was also manipulated. An example of the types of stories used is found in (16).

(16) The customer shouted angrily at the waitress that tie meal was awful. The waitress/she was new at the job and did not know' how to respond. (High plausibility) The waitress/she hoped the food would be better next time. (Equally plausible) The customer/she was still mad and threw the food at the chef. (Low plausibility)

Stories were presented auditorily and subjects were required to recall the story and verify the participants in each sentence (e.g. "Who was new at the job and did not know how to respond?"). Overall, aphasics were significantly better at assigning referents for repeated noun phrase anaphors than for pronouns. As well, responses were more accurate to high 
plausibility items than to low (percentage of errors according to plausibility: $22 \%$ - high; $37 \%$ - equal; $40 \%$ - low). The authors reasoned that resolution of the anaphor in high plausibility sentences could be done based on the use of world knowledge in addition to cues contained in the text. In contrast, resolution of the anaphors in the low and equal plausibility conditions depended upon the use of the contextual cues of the text. Therefore, the authors interpreted the results as suggesting that aphasics have difficulty using contextual linguistic information to resolve anaphors.

It is interesting to note, however, that the normal subjects also had increasing difficulty identifying the referents of pronouns as the plausibility of the situation decreased (percentage of errors according to plausibility: $3 \%$ - high; $6 \%$ - equal; $10 \%$ - low). While overall the aphasic group made more errors than the normals, it would have been interesting to test for an interaction in order to determine whether or not the difference between conditions was greater for the aphasic patients. Perhaps aphasics were not specifically impaired in their ability to use the linguistic context in order to resolve pronouns when compared to normals. Unfortunately, the authors did not include the data from both groups in the same analysis.

Thus, while LBD individuals have been found to use contextual information to a considerable degree to aid in language processing, there is some suggestion that they may be impaired in the ability to use contextual information to support preferred referents (Chapman \& Ulatowska, 1989; Grober and Kellar, 1981).

\subsection{Language deficits following right brain-damage}

The two preceding sections have established that contextual information is important in normal language processing (including anaphor resolution) and in aiding LBD individuals to compensate for some of their linguistic deficits. The following section will review the language deficits that emerge as a consequence of right brain-damage. Particular emphasis will be given to detailing how the evidence suggests that, contrary to non brain- 
damaged and left brain-damaged individuals, right brain-damaged individuals have particular difficulty in using contextual information.

The current view of the communicative profile of $\mathrm{RBD}$ individuals is that, apart from the existence of some subtle semantic deficits (Cappa, Papagno, \& Vallar, 1990; Critchley, 1962; Diggs \& Basili, 1987; Eisenson, 1962; Gainotti, Caltagirone, \& Miceli, 1983; Gainotti, Caltagirone, \& Miceli, 1979; Gainotti, Caltagirone, Miceli, \& Masullo, 1981; Joanette, Goulet, \& Le Dorze, 1988; Joanette, Lecours, Lepage, \& Lamoureux, 1983; Lesser, 1974; Villardita, 1987; Villardita, Grioli, \& Quattropani, 1988; Weinstein, 1964) and subtle sentence processing deficits (Basili, Diggs, \& Rao, 1980; Caramazza, Gordon, Zurif, \& DeLuca, 1976; Cavalli, De Renzi, Faglioni, \& Vitale, 1981; Coughlan \& Warrington, 1978; Grossman, 1982; Hier and Kaplan, 1980; Joanette et al., 1983;

Schneiderman \& Saddy, 1988; Swisher \& Sarno, 1969; Ulatowska \& Baker, 1976), their basic linguistic abilities at the levels of syntax, semantics, and phonology are relatively intact. The primary language deficits that result as a function of right brain-damage are instead related to discourse level phenomena.

\section{Narrative deficits:}

Significant deficits at the narrative level of discourse have been found to be characteristic of right hemisphere damage. A consistent finding is that RBD patients are deficient in their abilities to integrate story details (Gardner, Brownell, Wapner, Michelow, 1983; Wapner, Hamby, and Gardner, 1981) possibly due to a deficit in the organization of linguistic entities at this level (Hough, 1990). Such difficulty has been evidenced in a number of tasks. For example, RBD patients have been shown to have difficulty producing the moral of a story (Gardner et al., 1983; Wapner et al., 1981). Of the RBD patients tested, $50 \%$ erred on the production of the moral as compared to $30 \%$ of the normals (aged 45-65 years). However, it should be noted that an even greater percentage (56\%) of normal elderly subjects (aged 65-85 years) than RBD subjects produced errors on this task. However, the nature of the errors produced by the normal elderly subjects was 
different from those produced by RBD patients. According to the authors, the most striking difference was that RBD patients tended to be literal in their productions of the morals of stories (e.g. "take care of your tools"), whereas the normal elderly subjects tended to personalize their accounts, while remaining moralistic (e.g. "if you keep busy and active you won't become bored").

Difficulty identifying central themes of sto-ies has also been demonstrated by RBD patients (Hough, 1990; Moya, Benowitz, Levine, \& Finklestein, 1986), particularly when events are presented in a noncanonical form - for example presenting the central theme of a narrative at the end of a story, as in Hough (1990). Hough (1990) has interpreted this difficulty as evidence for an imjairment in the use of the macrostructure of a story, which is important in establishing global coherence (Kintsch \& van Dijk, 1978; van Dijk \& Kintsch, 1983). It should be noted, however, that this interpretation may be challenged by the results of Brookshire and Nicholas (1984). They found that in a task requiring subjects to answer true or false questions about auditorily presented short stories, RBD patients exhibited the normal pattern of recall by remembering the main ideas of the stories better than the details. Although not directly stated by the authors, such a result would seem to indicate a certain sensitivity to the macrostructure of the text. A possible explanation for the discrepancy in these results relates to the size of the subject groups used. In Brookshire and Nicholas (1984) only five RBD patients and five normal controls were used. Perhaps, therefore, the size of the groups may not have been sufficiently large to provide for adequate statistical power to detect group differences. Also, as Hough (1990) pointed out, the discrepancy in results may be attributed to the redundancy in the stimuli. She noted that Brookshire and Nicholas (1984) suggested that their brain-damaged subjects performed well because the text was redundant. In contrast, according to Hough (1990), the stimuli she used were intentionally not redundant.

Further indications that RBD patients have difficulty appreciating the "gestalt" nature of narratives has been found in their retellings of stories (Gardner et al., 1983; 
Wapner et al., 1981). Instead of paraphrasing the story being recalled as done by normals, RBD individuals frequently repeated parts of the story verbatim. As well, the RBD patients differed from normals in terms of their reactions to bizarre elements of the stories.

Whereas normal controls, as well as aphasics, evidenced a puzzled reaction to these elements and either failed to report them, disputed them or normalized them in their reports, RBD patients exhibited no reaction to their bizarre nature and included them in their recall of the story, frequently trying to justify them. Another important characteristic to note in the retelling of stories by RBD patients is the high frequency of embellishments and confabulations (Gardner et al., 1983; Herzyk, 1989; Wapner et al., 1981). This characteristic has been noted in spontaneous discourse as well. It is important to keep in mind that in Gardner et al. (1983) and Wapner et al. (1981), no formal statistical tests were reported, therefore, interpretations of the results are based solely on descriptive measures.

It has also been suggested that RBD individuals are impaired in the organization of narratives. For example, using a story recall task, Gardner et al. (1983) found that RBD patients made more sequencing errors than matched normal controls, normal elderly controls and aphasics. However, two points with respect to this result should be highlighted. First, the mean number of sequencing errors produced by the RBD group (defined as "each time a subject backtracks or violates the temporal order of events") was relatively low (1.5). Second, the incidence of sequencing errors for the RBD group was nearly equivalent to that of the normal elderly controls (1.1). Since, as just indicated, this set of investigations reported no formal statistical analyses, there is no statistical evidence to suggest that the number of sequencing errors made by RBD patients differed from those made by normal elderly controls or, for that matter, from age matched controls (0.5).

The results of performances on more specific tasks aimed at assessing organizational skills at the narrative level have also been interpreted as evidence that RBD patients have difficulty in appreciating the organization of narratives. In particular, it has been demonstrated that RBD patients are impaired in their ability to arrange sentences into 
paragraphs (Delis, Wapner, Gardner, \& Moses, 1983); Gardner et al., 1983;

Schneiderman, Murasugi, \& Saddy, 1992; Wapner et al., 1981). In Delis et al. (1983), for example, subjects were first presented with an initial sentence (in written form) which provided an introduction to the general theme of the story. Next, they were provided with five other sentences (also in written form) and required to arrange them into a story. The results indicated that $\mathrm{RBD}$ patients correctly arranged significantly fewer paragraphs than normals (mean percentage: 50 versus 83 , respectively). The results of these studies, however, should be contrasted with those of Huber and Gleber (1982) who found that RBD patients did not differ significantly from normals on their performance on a story arrangement task either when it was presented in pictorial or written form.

It is also of interest to note that Delis et al.'s (1983) interpretation of their results may be qualified by observations by Joanette et al. (1990) concerning the nature of the stimuli used. Delis et al. (1983) used three types of paragraphs in their task -- spatial, temporal and categorical -- and predicted, based on the knowledge that RBD individuals often exhibit visuospatial difficulties (Benton, 1985), that they would be more impaired on the spatial paragraphs than on the others. Contrary to their expectations, however, it was found that the RBD group were most impaired in organizing the sentences for the categorical paragraphs, followed by spatial and then by temporal. The authors offered no explanation for this finding. However, Joanette et al. (1990) offered an interesting explanation by suggesting that the different types of paragraphs place different demands on the subject. They explained that the temporal paragraphs are the simplest because correct organization may be done on the basis of lexical knowledge (e.g. knowing that "morning" comes before "noon"). The spatial paragraphs are more difficult than the temporal ones because they require an understanding of the whole sentence in relation to the various prepositions used. Finally, the categorical stories are probably most difficult because they require the subject to use inferential information based on the sentences in order to impose some organizational structure (e.g. knowing that more familiarity between a dog and its 
master is depicted by the sentence "The dog accepted the caress" than by "The dog is beginning to stay in the same room as its master"). This explanation of the differential difficulty exhibited by RBD patients with the various types of paragraphs is very plausible given the apparent difficulty RBD patients have with making inferences (Brownell, Potter, Bihrle, \& Gardner, 1986). Evidence for such a deficit will be expanded upon later. In light of this qualification, a pressing question regarding the contention that RBD patients have a reduced sensitivity to the organization of narratives concerns whether observed deficits at this level are not instead a function of impaired processes required to impose structure on narratives (such as ability to generate inferences or to relate linguistic entities to one another). At present, the answer to this question remains unresolved, but clearly warrants further attention.

Schneiderman et al. (1992) attempted to qualify the nature of the narrative deficit exhibited by RBD patients in story arrangement tasks. They noted that a possible reason for the discrepancy in results between Delis et al. (1983) and Huber and Gleber (1982) relates to the issue of macrostructure. They observed that Delis et al. (1983) used an initial sentence to set the theme of the story, whereas Huber and Gleber (1982) did not. Schneiderman et al. (15:22) proposed, therefore, that RBD patients differed from normals and LBD controls in their use of this information, indicating that the deficit lies in the use of the macrostructure of a text. To test this hypothesis they compared the abilities of RBD patients, LBD patients and normal controls to arrange sentences into stories under two different conditions -- Theme and No Theme. Under the Theme condition, the initial sentence served to provide a summary of the story to be arranged. The results indicated that there was no significant difference between groups in terms of percentage correct under the No Theme condition. However, under the Theme condition, the RBD group performed more poorly than both the normal controls and the LBD group, who did not differ significantly from each other. Thus, it appears that the story arrangement task was equally difficult for RBD patients, LBD patients and normal controls in the absence of structural 
cues. However, when cues concerning the organizational structure of the story were available (in the form of a theme-setting sentence), RBD patients, unlike normals and LBD patients, were unable to utilize this information. These results thus supported the hypothesis that $R B D$ patients were impaired in their ability to use macrostructural information (consistent with the view of Hough (1990)). It should be noted, however, as Schneiderman et al. (1992) pointed out, that the proposal of a deficit in the use of macrostructural information is quite general and needs to be more precisely defined. However, at the very least, these results once again suggest that at some level RBD patients have difficulty in using contextual information to process language.

Finally, with respect to narrative level processing deficits, a selective difficulty in the processing of emotional components in linguistically based tasks has also been found to be characteristic of right brain-damage. Deficits in this domain have been demonstrated at all levels of language processing -- ranging from the level of the word (Borod, Andelman, Obler, Tweedy, \& Welkowitz, 1992) to that of the sentence (Borod et al., 1992; Cicone, Wapner, \& Gardner, 1980) and finally to the narrative level (Bloom, Borod, Obler, \& Gerstman, 1992; Brownell, Carroll, Rehak, \& Wingfield, 1992; Gardner et al., 1983; Ostrove, Simpson, \& Gardner, 1990; Rehak, Kaplan, Weylman, Kelly, Brownell, \& Gardner, 1992; Wapner et al., 1981; Wechsler, 1973). Interestingly, the deficits exhibited by RBD patients with respect to emotional components of narratives do not appear to be absolute. For example, Wapner et al. (1981) found that although their RBD subjects were not able to attribute the correct emotions to the characters in the stories, given a particular context, the emotions they provided were, in fact, plausible. Similar results were found by Cicone et al. (1980) using a picture task. In contrast, Rehak et al. (1992) found that RBD patients were equally capable as controls at judging the emotions of the characters in the stories they used. Rehak et al. explained that the discrepancy between their findings and those of others (e.g. Cicone et al., 1980; Wapner et al., 1981) may be accounted for by the fact that in their stories one main character was developed throughout the story, perhaps 
allowing the patients to focus more fully on the protagonist's feelings. Ostrove et al. (1990) also found that RBD patients differed from normals in their assessment of how the person in the story was feeling, but only in being "overly positive" in their attributions of emotions.

In terms of discourse production, the analysis of the performance of RBD individuals on productive narrative tasks has also revealed characteristic deficits. The key finding among all the studies which specifically assessed the verbal expression of these patients is that while the number of words used by RBD individuals is not significantly different than that used by normals, the content of the narrative is considerably impoverished (Bloom et al., 1992; Diggs \& Basili, 1987; Joanette \& Goulet, 1990; Joanette, Goulet, Ska, \& Nespoulous, 1986). Specifically, the number of propositions produced by RBD individuals is considerably fewer than that produced ty normals resulting in what has been described as "empty speech" (Diggs \& Basili, 1987).

To conclude, the tentatively emerging view of the narrative abilities of RBD patients is that they have difficulty in appreciating the whole of the narrative and in integrating the various elements of the discourse. Such difficulties are manifested in an impaired ability to extract the morals of stories, identify central themes, appreciate emotional elements, organize sentences into paragraphs and appreciate and react appropriately to noncanonical elements of stories. It should be noted, however, that to view RBD individuals as presenting with an absolute narrative deficit would be misleading for, among other things, they have demonstrated a sensitivity to the canonical aspects of story processing (Rehak et al., 1992; Roman, Brownell, Potter, Seibold \& Gardner, 1987) and conversations (Rehak, Kaplan \& Gardner, 1992). For example, Roman et al. (1987) have found evidence to suggest preserved knowledge for scripts (routine sequences of steps for a particular activity) in RBD patients. Similarly, Rehak et al. (1992) have found that RBD patients do not differ significantly from normals in the processing of "structural" aspects of narratives. For the canonical stories they used (i.e. suspense stories as opposed to surprise), the RBD 
patients were efficient at remembering details and predicting outcomes indicating that "...by no means have they (RBD patie-ts) lost the ability to use a story framework in making sense of a text" (Rehak et al., 1992, p. 331). Note that these results challenge the contention that RBD patients are impaired in their use of the macrostructure of a story (Hough, 1990; Schneiderman et al., 1992). Perhaps the difference lies in the "familiarity" of the story framework -- more familiar canonical stories (e.g. a roller coaster collapsing) and routinized scripts versus novel stories.

As well, it is important to emphasize that many of the studies identifying narrative deficits in RBD individuals are either descriptive in nature (e.g. Gardner et al., 1983; Wapner et al., 1981) or have been challenged by contradictory results from other studies. Thus, it is clear that the details characterizing the processing of narratives by RBD individuals require further specitication and refinement. Additionally, questions concerning the possible nature of the deficits (for example, difficulty using contextual information) must be more formally addressed.

\section{Deficits in the processing of non-literal language:}

Indirect speech acts involve statements or questions whose meaning is not intended to be the literal meaning of the words chosen. For example, when a person says to another, "Can you pass the salt?", the true intent of the question is not to query the other person's ability but rather to request, "Please pass the salt". Accurate interpretation of an indirect speech act requires that the listener be sensitive to certain factors involved in the communicative exchange. In particular, a sensitivity to the situational and linguistic context is needed. Moreover, the listener must be able to interpret what is said in light of these factors. Given the narrative profile of RBD individuals detailed above, in particular their apparent difficulty in appreciating the "gestalt" nature of discourse, it is not surprising that RBD individuals have also been found to exhibit an impairment in interpreting indirect speech acts (Foldi, 1987; Foldi, Cicone \& Gardner, 1983; Heeschen \& Reisches, 1979, reported in Foldi et al., 1983; Hirst, LeDoux \& Stein, 1984; Molloy, Brownell \& Gardner, 
1990; Weylman, Brownell, Roman \& Gardner, 1989). The most consistent finding among these studies was that, in judging the appropriateness of a response to an indirect request (Foldi, 1987; Hirst et al., 1984) or in choosing an appropriate continuation following an indirect request (Weylman et al., 1989), RBD individuals showed a significant tendency to favor the literal interpretation over the non-literal one. Importantly, Weylman et al. (1989) demonstrated that the impairment did not exist solely at the level of interpreting visual representations (e.g. pictures or videos) as in Foldi (1987) and Hirst et al. (1984). Due to the possibility of visuo-spatial difficulties in RBD patients (Benton, 1985), results based on pictorial stimuli frequently entail the extra confound that a particular deficit may be attributed, at least in part, to the nature of the stimuli. Weylman et al. (1989) averted this possibility by using auditorily presented material (Task 1) supplemented by a written version (Task 2) and still found that RBD patients preferred the literal interpretation of the indirect requests. Therefore, based on results using both pictorial and written stimuli, there is converging evidence to suggest that $\mathrm{RBD}$ patients have a tendency to prefer the literal over the non-literal meaning in the interpretation of indirect speech acts.

Verbal irony is another form of non-literal language whose interpretation requires the listener to integrate knowledge of the situation with the actual utterance used in order to accurately interpret intended meaning. Verbal irony differs from indirect speech acts mainly in that verbal irony hinges on the use of "affective content" (Molloy et al., 1990). So, for example, sarcasm as a form of verbal irony, may arise when what is said is inconsistent with a particular action (e.g. Saying, "You're a great tennis player", when the person clearly is a poor tennis player). Note, however, that interpretation of an utterance as sarcastic depends upon the relationship of the people involved. If the two people involved in the conversation are friendly, the above utterance may be interpreted as joking or as a white lie. In other words, accurate interpretation of verbal irony depends not only upon an appreciation of the situational context, but also of the relationship between the people involved in the verbal exchange (Molloy et al., 1990). Kaplan, Brownell, Jacobs and 
Gardner (1990) investigated RBD individuals' abilities to deal with verbal irony. They presented subjects with short vignettes detailing a situation (such as a golf game) and the relationship between the participants (friendly or hostile). A target utterance (e.g. "You sure are a good golfer") ended the vignette and it was either congruent or incongruent with the situation depicted. The subjects were required to assess the intent of the utterance. It should be noted that the possible effect of intonation was controlled in this study. The target utterance was produced with no change of inflection for the sarcastic situations. The authors hypothesized that RBD patients would have difficulty appreciating the relationship between the discourse participants in evaluating the intended meaning of utterances. The results supported this hypothesis. When the target utterance was congruent with the situation depicted, there was no difference between the normal controls and the RBD patients. However, when it was incongruent with the situation depicted, RBD patients had significantly more difficulty than normals recognizing the intent of the speaker as joking, sarcastic, or a white lie relative to the relationship of the participants involved in the exchange. Similar resuits were found by Brownell et al. (1992).

Metaphors and idioms constitute the third tyje of non-literal language that has been investigated with respect to right hemisphere processing. In view of their difficulty in interpreting indirect speech acts and verbal irony, one would expect that RBD patients would similarly have difficulty with the processing of metaphors and idioms. In point of fact, research in this area has been equivocal. Some studies have, indeed, produced results to suggest that $\mathrm{RBD}$ patients are impaired in their interpretation of metaphors or connotative aspects of words (e.g. Brownell, Potter, Michelow \& Gardner, 1984; Brownell, Simpson, Bihrle, Potter \& Gardner, 1990; Bryan, 1988; Gardner \& Denes, 1973; Winner \& Gardner, 1977) and idioms (Myers \& Linebaugh, 1981; Van Lancker \& Kempler, 1987). On the other hand, other studies have found no difference between normals and RBD patients in the appreciation of the non-literal meaning of such utterances (e.g. Stachowiak et al., 1977; Tompkins, 1990; Tompkins, Boada \& McGarry, 1992). Discrepancies among 
the studies may be attributed to a number of factors. First, many of the studies used pictorial stimuli; the subject was required to point to the picture that best went with a particular metaphor or idiom. Obviously, possible visuo-spatial difficulties may have contributed to difficulties using pictures. Joanette et al. (1990) pointed out another important factor that one must consider when using pictorial stimuli -- the "degree of plausibility" of the picture depicted. They noted that for some non-literal expressions the literal and non-literal meanings are closely related; whereas for others they are not. They drew from an example found in Stachowiak et al. (1977) to illustrate their point. For the expression "the others strip him right down to his shirt" (translated from German) the metaphoric meaning of "take all of somebody's money" is close to the literal meaning. In contrast, for an expression such as "he filled his soup with pieces of bread" (translated from German), whose metaphoric meaning is "he got himself into a nice mess", the literal and metaphoric meanings are not obviously related. Joanette et al. (1990) cautioned, therefore, that "...idiomatic expressions do not all have the same status when their literal meanings are represented in pictures" (p. 185).

A second factor that must be considered when attempting to account for discrepant results found in the literature on metaphors and idioms concerns the use of "frozen metaphors" (i.e. common expressions such as "have a heavy heart"). Frozen metaphors are arguably part of semantic memory (Joanette et al., 1990) and, therefore, may be processed differently from truly non-literal language. Such an observation may help to account for the discrepant findings in Winner \& Gardner (1977) between the picture and explanation tasks they used. Contrary to the findings with the picture task, when subjects were asked to explain the meaning of metaphoric expressions, RBD patients were similar to normals in providing metaphoric as opposed to literal explanations. However, such an account fails to explain why Myers and Linebaugh (1981) and Van Lancker \& Kempler (1987) found RBD patients were impaired in their comprehension of idiomatic expressions. Presumably frozen idiomatic expressions should share a similar status in semantic memory 
(although difficulty may have been related to the task of picture matching as in Winner and Gardner (1977)). It should also be noted that Myers and Linebaugh (1981) addressed the discrepancy between their results and those of Stachowiak et al. (1977) by pointing out that the meaning of the idioms they used were explicitly explained in the text; therefore, better performance by the RBD patients in the Stachowiak et al. (1977) study may have been related to the redundancy of information present.

Another possible explanation to account for the discrepant findings among studies concerns on-line versus off-line tasks. Tompkins (1990; Tompkins et al., 1992) has argued that the findings of impaired processing by RBD individuals of metaphors and idioms using off-line tasks may reflect other impairments such as allocation of attentional resources, rather than a true deficit in the appreciation of non-literal meanings. In a lexical decision task, Tompkins (1990) found that prime words related to either the metaphoric or literal meanings of the target words were both useful in priming responses by RBD patients, thus demonstrating a sensitivity to the metaphoric meanings of the words. Similarly, Tompkins et al. (1992) found that RBD patients were sensitive to the meanings of idiomatic expressions in a word monitoring task. Her results suggested that, in fact, familiar idiomatic expressions are "processed in a manner simiiar to discrete lexical entries" (p. 634) -- an interpretation comparable to that provided for frozen metaphors. Given the discrepant findings in the literature concerning this area, it remains unclear to what extent the right hemisphere is involved in the processing of metaphors and idioms. Certainly, it appears that the familiarity of the phrase is an important factor in determining the presence or absence of impairments at this level in RBD patients.

Finally, it should be noted that some RBD individuals have been shown to exhibit difficulty in the interpretation of proverbs (Hier \& Kaplan, 1980). However, this difficulty was not found to be characteristic of all RBD patients tested. In fact, of the 32 RBD patients tested, 11 had scores that were higher than the mean for the normal controls. 
Overall, there is corvverging evidence to suggest that the right hemisphere is involved at some level in the processing of non-literal language. A key aspect of this involvement appears to be related to the ability to appreciate the situational and linguistic contexts in which non-literal language must be interpreted. The evidence suggests that RBD individuals have a certain deficit in this area.

\section{Deficits in the processing of humor:}

Individuals with right hemisphere damage have been shown to have difficulty appreciating humorous material. Early indications of such a deficit were provided by Gardner, Ling, Flamm and Silverman (1975). They found that in a task requiring subjects to choose the funniest cartoon out of a choice of four, RBD patients reacted qualitatively differently to the cartoons as compared to LBD patients and normal controls. While both brain-damaged groups were significantly impaired in their choice of the funniest cartoon compared to normals, the nature of LBD patients' responses more closely mirrored the normal pattern. RBD patients, on the other hand, produced "bizarre" responses to the cartoons by producing extreme reactions -- either by laughing excessively or by not laughing at all.

Since the Gardner et al. (1975) study, a number of studies (e.g. Bihrle, Brownell, Powelson, \& Gardner, 1986; Brownell, Michel, Powelson \& Gardner, 1983; Dagge \& Hartje, 1985; Gardner, 1981; Gardner et al., 1983; Wapner et al., 1981) have sought to qualify the deficit in processing humor exhibited by RBD patients. The results of these

studies have indicated that the deficit RBD patients have in understanding humor appears to be related to a distinction between the formal aspects of humor (i.e. recognizing incongruency or surprise) on the one hand, and interpreting this incongruency within a context to arrive at humor, on the other hand. In particular, RBD individuals have been found to be sensitive to the surprise aspect of humor. For example, when required to choose the punch lines to jokes, RBD patients were found to prefer the incorrect nonsequitor endings -- those that were surprising but irrelevant to the body of the joke 
(Brownell et al., 1983; Wapner et al., 1981). Thus, it appears that while the RBD patients are impaired in their ability to relate an incongruity to a context (which would result in humor), they are capable of recognizing that incongruity is a necessary aspect of humor (Joanette et al., 1990).

Possible explanations for language deficits following right brain-damage:

As we have seen, the communicative profile of $R B D$ patients involves a number of discourse level deficits. While these discourse level phenomena are quite distinct, a number of investigators have attempted to postulate a common underlying deficit to account for the impairment at this level.

Gardner et al. (1983) and Wapner et al. (1981), for example, have suggested that RBD patients have difficulty with what they term their "plausibility metric"; that is, RBD patients are unable to assess the plausibility of certain elements in relation to the whole of the narrative. In addition to an impaired "plausibility metric", it has been proposed that RBD patients have difficulty using the context (both discursive and situational) in evaluating linguistic entities. Cook (1989a) also supports this position and has argued that the right hemispher is fundamentally involved in interpreting language within a context (see also Prather, Gardner \& Brownell, 1989; Cook, 1989b) .

This proposed difficulty in using contextual information follo damage may have consequences for the ability to make inferences, since this ability presumably requires an appreciation of context. Indeed, a deficit at this level has also been proposed to explain the narrative difficulties exhibited by RBD individuals. However, it is not clear that "pure inferencing" deficits, in terms of an absolute inability to generate inferences, exist in this population. Consider, for example, the study by Brownell et al. (1986). They presented subjects with two sentences which were to be read as a unit and required the subjects to accept or reject an inference based on the presentation of these two sentences (e.g. Sally brought a pen and paper with her to meet the famous movie star. The article would include comments on nuclear power by well-known people). The sentences 
were constructed such that when read together they encouraged a particular interpretation, deemed the correct inference. However, when one of the sentences was read alone it made another interpretation more likely, resulting in an incorrect inference. For instance, with reference to the preceding example, when a person meets a movie star with a pen and paper the most common inference is that the person wants an autograph. This sentence occurred in both the first and second positions an equal number of times.

Results indicated that the RBD group had more difficulty than normals on the inferencing questions. However, upon closer inspection of the data, it was found that the inferencing deficit could primarily be accounted for by a tendency of the RBD patients to accept false inferences as true. When looking at the responses to correct inferences, no obvious deficit on the part of the RBD group was evidenced. Moreover, it should be noted that RBD patients demonstrated a tendency to make more errors, on both correct and incorrect inferences, when the sentence containing misleading information was in the first position. As noted by the authors, such a finding suggests that RBD patients may simply have more difficulty than normals in rejecting an initial interpretation. This inability of RBD individuals to reject contradictory elements has been observed previously (Wapner et al., 1981) and appears to parallel apparent sentence processing deficits observed by Schneiderman and Saddy (1988) who found that RBD individuals had difficulty reassigning the syntactic status of a word, as well as the results of Ulatowska and Baker (1976) who found that RBD patients had difficulty rearranging words in an anagram task to form a different correct arrangement, once a first solution was found.

Similar results to those of Brownell et al. (1986) have also been found by McDonald and Wales (1986). Using a recognition task, they also found no difference between RBD patients and normals in the acceptance of true inferences, but more of a tendency for RBD patients to accept false inferences as well. The authors addressed the possibility that this pattern of responding was indicative of a possible positive response bias, but dismissed it claiming that a positive response bias would be reflected in higher 
correct responses as well. Further evidence for intact inferential abilities in RBD patients has been found by others as well (Brookshire \& Nicholas, 1984; Joanette \& Goulet, 1987. cited in Joanette et al., 1990; Tompkins, 1991; Tompkins \& Mateer, 1985).

Overall, it appears that RBD patients have preserved inferential abilities, at least at some level. Joanette et al. (1990) have suggested that inferencing difficulties by RBD patients emerge only when many possible interpretations are contained within the context. Such an explanation is consistent with the findings of Tompkins and Mateer (1985) who found that RBD patients only exhibited inferencing difficulties when the paragraphs upon which the inferences were made contained conflicting/inconsistent information (e.g. a paragraph detailing the unhappiness of a boss because of declining sales which concluded with the statement, "They're [his employees] doing a wonderful job"). When the information was consistent and redundant, no inferencing difficulties were found. This notion that redundancy of information affects inferencing abilities was also supported by the results of Tompkins (1991), who found that semantic redundancy significantly improved the ability of RBD individuals to infer emotions.

\section{Summary:}

Over the past thirty years, the task of more precisely defining the language deficits associated with right hemisphere brain-damage has been the primary purpose of a number of investigations. The compilation of results of these studies has suggested a communication profile of RBD patients that is characterized by the existence of discourse level impairments - primarily in the processing and production of narratives, interpretation of non-literal language and appreciation of humor - with seemingly intact syntactic and phonological skills accompanied by subtle semantic and sentence processing deficits.

These investigations have been crucially important in empirically establishing the existence of language deficits in RBD patients and, thereby, alerting language researchers to the important role of the right hemisphere in language processing. Moreover, based on the results from studies investigating a number of different discourse level phenomena (e.g. 
narratives, non-literal language, humor), a recurrent theme has emerged that has provided insight into the nature of the communication deficit exhibited by RBD individuals. The common thread among most accounts is an apparent difficulty in appreciating the gestalt nature of discourse and, relatedly, in interpreting linguistic entities within the situational and discursive context of the exchange.

Right brain-damage and pronoun resolution:

It is surprising to note that, given the important role that pronoun resolution plays in discourse processing and the existence of discourse level deficits as a function of right brain-damage, little research has been conducted on the ability of RBD patients to resolve pronouns. Lesser (1986) touched on this issue and found that RBD individuals were impaired in their ability to identify the correct referent of a pronoun in coordinated sentences linked by "and he" (e.g. Tom lent Jim some money and he bought a car). The pronoun in the second clause was thought to be disambiguated by the semantics of the verb. As such, Lesser argued that difficulty in pronoun assignment reflected a semantic impairment. However, given the apparent difficulty of RBD individuals in using contextual information, it is equally likely that the semantics of the verb were understood by the patients and that the difficulty lay in using the knowledge from the first clause in disambiguating the pronoun in the second clause.

A more recent study by Brownell et al. (1992) found that RBD patients were indeed capable of resolving pronouns in integrating sentences. The results also suggested that they depended upon this linguistic ability to a greater degree than did normals. Subjects were auditorily presented with vignettes such as (17) and required to evaluate the meaning of responses such as (17a), (17b), (17c) or (17d). Note, "Rick" is represented as a 10year-old boy. Note, also that "he" is unambiguous based on gender cues. 
(17) Mr. Perkins is happy with his wife. She brought home his favorite ice cream for desser. As she is serving the ice cream, Mrs. Perkins notices that their son Rick is no longer at the table. Mrs. Perkins asks her husband, "Where did Rick go?" Mr. Perkins replies...

(17a) "He's getting himself chocolate syrup from the pantry." [plausible, with pronoun]

(17b) "There's chocolate syrup in the pantry." [plausible, without pronoun]

(17c) "He's getting himself coffee from the kitchen." [implausible, with pronoun]

(17d) "There's coffee in the kitchen." [implausible, without pronoun]

Subjects were given the following four choices as to the meaning of the response and asked to choose one:

(18a) not a sensible answer to the question

(18b) an attempt to answer the question as asked

(18c) sarcastic and nasty

(18d) joking or in fun

Results indicated that when a pronoun was present in the response, regardless of the plausibility of it, RBD individuals demonstrated more of a tendency than normals to interpret the response as appropriate (i.e. "an attempt to answer the question as asked").

The sparse evidence available, therefore, suggests that RBD individuals are able to resolve anaphors when the resolution is constrained by linguistic factors. However, there is some suggestion that if resolution depends upon the integration of contextual information (as in Lesser, 1986), successful resolution may be impaired. 


\section{Chapter 2: Statement of the problem}

It has been proposed that the underlying deficit related to the discourse level impairments exhibited by RBD individuals involves their inability to effectively use contextual information to integrate linguistic entities and thereby understand the relations of utterances within a discourse. As pointed out earlier, such a proposal necessarily presupposes an important role for the use of context in discourse processing. In support of this proposal evidence has been reviewed that suggests that contextual information is influential in many aspects of normal language processing - ranging from the resolution of lexical and syntactic ambiguities to anaphor resolution. In addition, a considerable amount of evidence has been reviewed which suggests, in contrast to the proposed deficit by RBD individuals, that LBD individuals can, and actively do, use contextual information to help compensate for their linguistic deficits.

The identification of a common underlying deficit to account for a number of seemingly different language related deficits is useful in furthering our understanding of the role of the right hemisphere in language processing. However, while previous investigations have been instrumental in identifying the possibility of difficulty in using contextual information to process language following right brain-damage, it seems that precise tests of this hypothesis are lacking. Difficulty in the use of contextual information has really only been invoked as a logically possible explanation to account for the language deficits identified in $R B D$ patients. Many investigations have simply assumed that because success on a particular task requires a sensitivity to contextual considerations (e.g. the interpretation of indirect speech acts), failure on the task necessarily indicates difficulty in using contextual information. It is important to realize that while this may be the most likely explanation, it is not the only possible interpretation.

Based on the literature reviewed, not many studies have specifically contrasted the performance of RBD patients under different types of context conditions. Tompkins (1990; 1991; Tompkins \& Flowers, 1987; Tompkins et al., 1992) has been a primary force 
in attempting to manipulate the use of different types of contexts by RBD patients. However, of these studies only Tompkins and Flowers (1987) and Tompkins (1991) have provided a strong test of the hypothesis. Moreover, the results of these studies have suggested that RBD patients can use contextual information for certain aspects of language processing. In Tompkins and Flowers (1987), for example, it was found that RBD patients could use the information provided in a previous paragraph to facilitate the detection of mood (e.g. happy, angry, afraid, no emotion) of auditorily presented semantically neutral sentences (e.g. "What are you doing here?") compared to when the senterices were presented in isolation. Similarly, Tompkins (1991) found that the use of highly redundant story contexts improved the detection of mood to auditorily presented semantically neutral comments (e.g. "I should be there soon") as compared to the use of moderately redundant story contexts.

In contrast to Tompkins and Flowers (1987) and Tompkins (1991), the studies by Tompkins (1990) and Tompkins et al. (1992), while involving the manipulation of context, are not strong examples of direct tests of the use of contextual information by RBD patients. Tompkins (1990) only looked at the influence of word primes in a lexical decision task, although she did find an effect of context for RBD patients. In Tompkins et al. (1992), while context was manipulated, it did not really factor into the interpretation of the results and, therefore, was not a direct test of the use of contextual information by RBD patients. Using a word monitoring task, it was found that responses to target words were faster when presented in idiomatic expressions (e.g. "rat" in "smell a rat") than in neutral expressions (e.g. "see a rat"), when preceded by both contexts that biased the idiomatic meaning (e.g. "My lawyer was studying my contracts. When he smelled a rat, he warned me") and those that biased the literal meaning (e.g. "My cat was hunting one night. Wheri he smelled a rat, he attacked it") for all groups tested. Tompkins et al. (1992) interpreted these results as evidence that the idiomatic phrase was being processed as a unit. The point to note is that the key distinction was whether or not the target word was present in an 
idiomatic expression or a neutral one, not the type of context preceding it. In fact, the same results would probably have obtained regardless of the types of preceding contexts used. In this way, therefore, the results do not directly address the issue of the use of contextual information by RBD patients.

Another study that specifically manipulated the use of a preceding context versus no context in language processing was that of Schneiderman et al. (1992). In contrast to the findings of Tompkins and Flowers (1987) and Tompkins (1991), however, RBD individuals were found to be impaired in their use of contextual information to facilitate the arrangement of sentences into stories. Similarly, Weylman et al. (1989) also found that RBD patients were impaired compared to normals in their use of the biasing information in preceding contexts to determine a literal versus a non-literal reading of an indirect speech act.

In view of the paucity of research specifically addressing the issue of whether or not RBD individuals are able to use contextual information to process language, as well as the existence of contradictory results, it is clear that more rssearch aimed at resolving this issue is needed. Moreover, while a proposed deficit in the use of contextual information by RBD individuals is intuitively appealing in explaining their communicative deficits, it is important to qualify the nature of this deficit. For instance, interesting questions concern whether the deficit holds at the level of the sentence (as in the integration of information between clauses) or at the level of the most basic discourse -- two sentence passages. As well, it is of interest to know whether there is a distinction between the ability to use contextual information specifically stated in the discourse versus the ability to use contextual information based on general world knowledge. Finally, another important distinction relates to the ability of RBD individuals to use contextual information in specific contrast to their more basic linguistic processing abilities (e.g. syntax and semantics) which, apart from subtle incompetencies, appear largely intact. 
In an attempt to formally address these questions the following series of studies investigated the ability of RBD individuals to resolve pronouns. The resolution of pronouns was chosen as the linguistic process to investigate with this target population for two primary reasons. The first relates to the fact that, as noted earlier, pronoun resolution is important in discourse processing, and given that RBD individuals exhibit discourse level impairments, it seemed a natural candidate for study. Secondly, pronoun resolution is an interesting area of research with respect to the RBD population because, as we have seen, it can be influenced by both linguistic and contextual factors.

\section{Summary of the problem:}

To summarize, the purpose of this investigation was to help clarify the extent to which the use of contextual information in language processing is impaired as a consequence of right brain- $c$ mage. The resolution of pronouns was used as the linguistic vehicle by which to address this issue. The abilities of LBD individuals and non braindamaged controls, consisting primarily of older adults, to use contextual information to resolve ambiguous pronouns were also investigated. These two groups served as natural control groups for the RBD group in order to discount the possibility that any effects found were simply a result of brain-damage and/or aging. As well, the investigation of the LBD group was interesting in its own right in terms of corroborating and extending the view that contextual information aids language processing by aphasic individuals. 


\section{Chapter 3: General methods and hypotheses}

Three principal experiments were conducted. In all three experiments, subjects were required to explicitly indicate the correct referent of a pronoun. Reaction times and accuracy were recorded. The first two experiments explored the use of the implicit causality of verbs by RBD and LBD individuals and non brain-damaged (NBD) controls. This property of verbs was chosen as a target of investigation for two primary reasons. First, it has been shown to be influential in the resolution of pronouns by both NBD (Caramazza et al., 1977; Garvey \& Caramazza, 1974; Garnham et al., 1992; Garvey et al., 1976; Grober et al., 1978; Light \& Capps 1983, cited in Light \& Albertson, 1988; Vonk, 1985a; 1985b) and LBD (Grober \& Kellar, 1981) individuals and, therefore, it was of interest to determine whether or not RBD individuals would also be influenced by this semantic property of verbs. Second, and more importantly for the primary purpose of this investigation, it allowed for discussion conceming the use of contextual information at the level of the single sentence, in terms of the integration of information between clauses. This point will be elaborated upon in a moment.

Thus, the first experiment addressed the resolution of pronouns at the level of the single sentence. The second experiment used stimuli consisting of two sentences and thus addressed the abilities of subjects to use contextual information across sentences. The third experiment also used sentence pairs as stimuli, but differed from Experiment 2 in two important ways. First, in contrast to Experiment 2 (wherein the context was only supportive of a preferred referent, but was not necessary for correct resolution), in Experiment 3 successful resolution of the pronoun depended upon the interpretation of the leading sentence. The two experiments also differed with respect to the type of contextual information provided. In Experiment 2, the important information that was supportive of a particular interpretation of the pronoun was specifically stated in the context and was, for the most part, a restatement of the disambiguating information found in the pronominal sentence (e.g. Mark had an awful tennis serve and was a terrible player compared to Paul. 
Mark lost to Paul because he was a poor player). In contrast, in Experiment 3 the choice of a preferred referent crucially depended upon assessing the plausibility of an action based on a particular situation detailed in the context. The assessment of plausibility was based predominantly on the use of general world knowledge. For example in order to resolve the pronoun "he" in the sentence pair, "Henry went to the party while John stayed at the store. He danced with some women", it is important to know that "dancing" is more likely to occur at a party than at a store.

While specific hypotheses will be discussed during the presentations of the individual experiments, some general expectations may be offered. With respect to the first experiment, an effect of implicit causality was expected for all three groups tested. Normally, this effect would be evidenced in slower reaction times and possibly more errors to sentences in which the subordinate clause of the sentence (which contained the disambiguating information) provided an interpretation that was inconsistent with the verb's natural bias. This "normal" effect of implicit causality was expected for the NBD group. However, due to the necessity of integrating information between clauses for successful resolution of the pronoun, it was hypothesized that RBD patients may not exhibit the "normal" effect of implicit causality. This possibility was considered for the following reason. A reaction to the inconsistency of an interpretation to a verb's natural bias, in terms of a slower reaction time but a subsequent ability to successfully resolve the pronoun, requires that the interpretation of the subordinate clause to be made in relation to the event depicted in the first clause. Given a possible deficit in this ability, it was conceivable that RBD patients would not react to the inconsistency of the sentences and, thus not respond any more slowly to them. However, an effect of implicit causality may still be manifested in more errors to inconsistent sentences, if the RBD patients were choosing as the correct referent the preferred referent based on the verb's natural bias. It should be noted also that, given the results of Grober and Kellar (1981), LBD individuals 
might also be expected to fail to exhibit a "normal" effect of implicit causality and thus demonstrate a deficit in the ability to integrate information between clauses.

The second experiment explored the use of contextual information across sentences in resolving pronouns. RBD individuals, given their apparent difficulty in using contextual information, were not expected to show any effect of context. In contrast, both the NBD and LBD groups were expected to show an effect of context, in terms of shorter reaction times and possibly fewer errors to sentences which were preceded by a supporting linguistic context than to those sentences presented in isolation. However, given the results of Chapman and Ulatowska (1989), which suggested that aphasics have difficulty using contextual linguistic information to resolve anaphors, there was a possibility that the effect of context would not be as great for the LBD group as compared to the NBD controls.

It was expected that the results of Experiment 3, which investigated the use of pragmatic constraints on pronoun resolution, would confirm those of Experiment 2. That is, both the NBD and LBD groups were expected to show an effect of context, while the RBD group was not.

Four pilot studies were also conducted using only normal subjects (both younger and older adults). The purpose of these pilot studies was twofold. First, they were conducted in onder to refine the construction of the stimuli to be used in the principal experiments. Second, they enabled the investigation of possible age effects on the use of implicit causality and contextual information. It is important to note that the question of age differences with respect to these abilities was not a primary focus of this research. However, it was important to establish that performance on the tasks to be used was not primarily a function of age, as most of the individuals comprising the non brain-damaged control group were older adults. Thus, prior to embarking upon the investigation of the use of contextual information by brain-damaged individuals in resolving pronouns, it was first important to establish effects of implicit causality and context with the older adult group. If they did not exhibit these effects, then the possible finding that brain-damaged 
individuals (in particular RBD individuals) would not show these effects would not be very interesting, given that one might simply attribute a lack of effect to aging. The set of pilot studies will be presented and discussed prior to the presentation of the three principal experiments. 


\section{Chapter 4: Pilot Study 1}

The purpose of Pilot Study 1 was twofold. First, it was conducted in order to establish the direction of causality of the verbs to be used in Experiments 1 and 2. Second, it was conducted in order to address possible effects of aging on the property of implicit causality of verbs. Since older adults were to be used in the principal experiments, it was important to establish that the direction of causality of the verbs to be used was the same for both older and younger adults.

Method

Subjects. Two groups of subjects were tested. The first group, the Younger Adult (YA) group, consisted of 30 university students between the ages of 17 and 30 years. The second group, the Older Adult (OA) group, consisted of 30 elderly individuals with postsecondary education between the ages of 60 and 80 years. All subjects were native speakers of English.

Materials. The stimuli used were based on those used in the Garvey et al. (1976) study and consisted of sentence fragments of the form NP1 past-Verb NP2 because Pronoun (e.g. John feared Bill because he...). The verbs chosen were based on the results of Garvey et al. (1976) as well as those of Caramazza et al. (1977). In both these studies, verbs were identified as either strong NP1 type verbs or strong NP2 type verbs. NP1 type verbs were defined as verbs which marked the surface structure subject as the cause of the action. Conversely, NP2 type verbs were defined as verbs which marked the surface structure object as the cause of the action. Nine NP1 type verbs (questioned, sold, lied to, confided in, confessed to, won, called, followed, approached) and eight NP2 type verbs (blamed, punished, criticized, gave, feared, scolded, praised, envied) were selected. Thirteen additional verbs (bored, interested, lost to, tailed, warned, rewarded, amazed, accused, trusted, admired, congratulated, encouraged, defended) whose direction of causality was unknown were also used resulting in a total of 30 test verbs. 
Sixty test items were used (two instances of each verb); in one item of the pair both NP1 and NP2 were female and in the other item both were male. No grammatical cues were available to aid in the assignment of the referent. Fourteen distractor items were also used in which the assignment of the referent was unambiguous based on gender agreement (e.g. John helped Susan because she...). The distractor stimuli were designed such that the correct antecedent was in the object position as often as it was in the subject position.

Procedure. Subjects were tested in small groups, consisting of one to five individuals at a time. As in the Garvey et al. (1976) study, they were told that the purpose of the study was to investigate how people ascribe reasons for actions. The experimenter read the sentence fragments orally to the subjects. The subjects were instructed to complete the sentence fragment with a written response by providing a reason for the action or emotion expressed in the first part of the sentence. They were encouraged to respond with the first thing that came to mind and to respond as quickly as possible. No time limitations were enforced. If a subject could not think of a response he/she was instructed to leave it blank. Repetition of the sentence fragments by the experimenter was allowed if requested by the subjects. Occasionally, subjects would remark on the fact that the pronoun was ambiguous. In those instances they were simply encouraged to complete the sentence with the first response that came to mind.

Results

The responses were scored by two judges. Their task was to decide to which antecedent (NP1 or NP2) the pronoun was assigned based on the completion of the sentence fragment provided by the subjects. If it was unclear as to which antecedent the pronoun was assigned, the response was scored as ambiguous (A). An error (E) was scored if the subject made an error in the completion of the sentence (for example, by changing the pronoun to "they") or if the subject failed to respond. A response was scored as illegible (I) if the judge could not read the subject's writing. In those instances where the judges disagreed, the item was scored as ambiguous. The percentage of responses 
across all the verbs that were scored as either A, E, or I was 13 for the YA group and 11 for the OA group. The percentage of dominant response (\%DR) was calculated following the formula provided by Garvey et al. (1976). The number of NP1 responses or NP2. responses for a particular item was divided by the total number of responses for that item, less the sum of ambiguous, error and illegible scores for that item and then multiplied by 100.

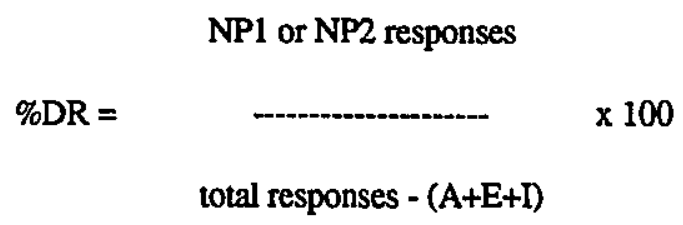

The calculations were done separately for the two groups. Of the 30 verbs tested, 25 had a \%DR equal to or greater than 70 for both groups and were judged as being either strong NP1 or NP2 verbs (see Table 4.1). This cutoff of 70\% DR was the same as that used by Caramazza et al. (1977). For the remaining 5 verbs, for at least one group, the highest percentage of dominant response, either for NP1 or NP2, was less than 70 and so these verbs were classified as neutral (see Table 4.2).

For the verbs used in the two previous studies (Garvey et al., 1976; Caramazza et al., 1977) the percentage of dominant response was as expected, with the exception of two verbs. In Garvey et al., the verbs blamed and gave were identified as NP2 verbs with a \%DR equal to 100 and 96 , respectively. The results of this study found the verb blamed to be associated with only a $65 \%$ and 59\% NP2 DR for the younger and older adult groups, respectively. Similarly, the verb gave was associated with only a 37\% NP2 DR for the younger adults and a 24\% NP2 DR for the older adults. It should be noted that in the Garvey et al. study the verb gave was not used in a sentence completion task but rather in a task requiring the subject to answer a question with 'because' (e.g. Why did the mother punish her daughter?). The discrepancy, therefore, concerning the results with the verb 
Table 4.1

Percentage of dominant response (DR) for strong NPl and NP2 verbs

$\begin{array}{llcc}\text { NP1 Verbs } & \begin{array}{l}\text { Younger } \\ \text { Adults (YA) }\end{array} & \begin{array}{l}\text { Older } \\ \text { Adults(OA) }\end{array} & \text { YA-OA } \\ \text { * bored } & 98 & & \\ \text { questioned } & 80 & 100 & -02 \\ \text { followed } & 74 & 85 & -05 \\ \text { approached } & 84 & 79 & -05 \\ \text { sold } & 86 & 94 & -10 \\ \text { lied } & 96 & 89 & -03 \\ \text { lost to } & 100 & 100 & -04 \\ \text { confided in } & 87 & 100 & - \\ \text { confessed to } & 97 & 80 & +07 \\ \text { won } & 92 & 98 & -01 \\ \text { tailed } & 92 & 96 & -04 \\ \text { * warned } & 81 & 90 & +02 \\ \text { * amazed } & 94 & 70 & +11 \\ \text { * interested } & 96 & 100 & -06 \\ \text { called } & 90 & 98 & -02 \\ & & 92 & -02\end{array}$

NP2 Verbs

- admired 100

- trusted 83

punished $\quad 92$

- rewarded 92

criticized 80

feared 83

envied 90

scolded 91

- congratulated 97

praised $\quad 80$

$\begin{array}{rr}100 & -- \\ 71 & +12 \\ 95 & -03 \\ 86 & +06 \\ 77 & +03 \\ 73 & +10 \\ 95 & -05 \\ 87 & +04 \\ 100 & -03 \\ 84 & -04\end{array}$

* Verbs not tested in previous studies. 
Table 4.2

\% Dominant Response (DR) for Neutral Verbs

\begin{tabular}{lll} 
& \multicolumn{2}{l}{$\begin{array}{l}\text { Younger } \\
\text { Adults (YA }\end{array}$} \\
& NP1 & NP2 \\
blamed & 35 & 65 \\
* accused & 39 & 61 \\
*ncouraged & 76 & 24 \\
* gave & 63 & 37 \\
defended & 70 & 30
\end{tabular}

\section{Older \\ Adults (OA)}

NP1 NP2

$41 \quad 59$

$67 \quad 33$

5941

$76 \quad 24$

$67 \quad 33$

*Verbs not tested in previous studies. 
gave may have been due to the nature of the task. With respect to the discrepancy of results with the verb blamed, however, no adequate explanation can be offered since blamed was used in the sentence completion task. It should further be noted that neither the verb gave nor the verb blamed was tested by Caramazza et al. (1977). These two verbs (blamed and gave), as well as three other verbs (accused, encouraged, defended) were those that were classified as neutral due to their weak dominant response. Of the thirteen verbs used whose direction of causality was unknown, six emerged as strong NP1 verbs (bored, lost to, tailed, warned, amazed, interested) and four as strong NP2 verbs (admired, trusted, rewarded, congratulated).

No obvious age related difference in percentage of dominant response for the various verbs was suggested by the results, although certain aspects of the results should be noted. Differences between the \%DR for the various verbs for younger and older adults were calculated by subtracting the \%DR associated with a particular verb provided by the OA group with the \%DR associated with the same verb provided by the YA group. These differences ranged from -10 to 11 for the NP1 verbs and -5 to 12 for the NP2 verbs and were calculated to ensure that the groups did not differ widely in terms of their responses to the implicit causality of the verbs. The important observation to note concerning these calculations was that the verbs that emerged as strong NP1 verbs or strong NP2 verbs, as defined by a \%DR equal to or greater than 70 , were the same across groups, with the exception of three (which were hence classified as neutral). Within the younger adult group the verbs encouraged and defended were found to be NP1 verbs with \%DR equal to 76 and 70, respectively. For the older adults, however, the \%DR for these verbs was only 59 and 67 , respectively. The verb gave, on the other hand, emerged as a stronger NP1 verb for the older adults (\%DR=76), than for the younger adults (\%DR=63). Discussion

As expected, support for the notion of the implicit causality of verbs, as measured by percentage of dominant response, was found. As in previous accounts, this semantic 
property of verbs emerged as a continuous measure with the percentage of dominant response for the verbs ranging from 59 to 100 . No obvious differences in the responses of older and younger adults to the implicit causality of verbs was noted. These results are consistent with those of Light and Capps (1983, cited in Light \& Albertson, 1988) who also found no significant difference in the abilities of younger and older adults to assign pronouns to referents using the information provided by the implicit causality of the verbs. 


\section{Chapter 5: Pilot Studies 2a and 2b}

\section{Pilot Study 2a}

As has been reviewed, considerable evidence has accumulated which posits an important role for contextual information in the resolution of pronouns (e.g. Hirst \& Brill, 1980; Light \& Capps, 1986; Stevenson \& Vitkovitch; Tyler \& Marslen-Wilson, 1982). The primary purpose of this study was to further investigate the role that contextual information plays in the resolution of pronouns by normal adults. More importantly, the interesting question concerned whether or not preceding contextual information could modulate the influence of a linguistic factor -- the implicit causality of verbs -- in the correct choice of an antecedent. Specifically, it was of interest to know whether or not contextual information could serve to attenuate or possibly nullify this effect of the verb on pronoun resolution. It was hypothesized that if contextual infcrmation can serve to constrain preferred choices of antecedents, then when a context was provided that was consistent with the disambiguating information found in the second clause, the consistency or inconsistency of the interpretation established to the verb's natural bias should no longer play a role. That is, the context should be sufficient to override the effects of the implicit causality of the verb. On the other hand, it was possible that even though contextual information may be useful in assigning a pronoun, the bias of the verb would persist and the effect of implicit causality would remain.

A secondary question relating to the effects of aging on the use of contextual information was also addressed by this experiment. There is some evidence to suggest that older adults have difficulty making inferences (e.g. Cohen, 1979; 1981; Light, Zelinski \& Moore, 1982; Zacks, Hasher, Doren, Hamm \& Attig, 1987; Zacks \& Hasher, 1988) and using contextual information in language processing (Hess, 1984; 1985; Hess \& Higgins, 1983; Light et al., 1982; Simon, 1979). Such difficulties would necessarily be reflected in problems using the information present in the leading sentence. Therefore, the second 
purpose of this experiment was to determine whether or not older adults were as sensitive as younger adults to the presence of contextual information in the resolution of pronouns. Method

Subjects. Two groups of subjects, different from those used in Pilot Study 1, were tested. The Younger Adult (YA) group consisted of 20 university students ranging in age from 19 years to 32 years (mean age $=24$ years). The Older Adult $(\mathrm{OA})$ group consisted of 20 elderly individuals with post-secondary education ranging in age from 61 years to 77 years (mean age $=69$ years). All subjects were right-handed and their native language was English.

Materials and_Apparatus. The stimuli used in this experiment were very similar and sometimes identical (except for the names), to those used in Caramazza et al. (1977) with one alteration -- the addition of a leading sentence for half of the stimuli. The leading sentence served to provide a context for the interpretation of the second sentence.

Thus, there were two factors that were manipulated in the creation of these stimuli. The first factor, Context, related to the presence or absence of a leading sentence. This factor had two levels - context consistent (CC) and no context (NC). In the CC condition, the information provided by the leading sentence was consistent with the reason given for the action or emotion expressed by the verb. It essentially provided a "restatement" of the disambiguating information present in the second sentence. In the NC condition, the position of the leading sentence was marked by a string of X's. The second factor, Verb Consistency, related to the consistency of the interpretation set up by the information in the subordinate clause of the second sentence to the verb's natural bias. It had two levels verb consistent (VC) and verb inconsistent (VI). The resulting combination of sentence pairs was as shown in Table 5.1 (see Appendix A for a complete listing of the stimuli).

Notice that the VC and VI sentences remain the same across the different context conditions for each verb ${ }^{1}$. It should also be noted that in the leading sentences both names were always mentioned and the names were mentioned in the same order that they appeared 
Table 5.1

Examples of Stimuli for Pilot Study 2a

Note that the verb used in these stimuli, confessed, is a NP1 type verb.

CC-VC Mark felt extremely guilty for cheating and desired to be pardoned by Paul. Mark confessed to Paul because he wanted forgiveness.

CC.VI Mark knew that Paul was an understanding priest and would grant absolution. Mark confessed to Paul because he offered forgiveness.

NC-VC XXXXXXXXXXXXXXXXXXXXXXXXXXXXXXXXXXXXX Mark confessed to Paul because he wanted forgiveness.

NC-VI XXXXXXXXXXXXXXXXXXXXXXXXXXXXXXXXXXXXX Mark confessed to Paul because he offered forgiveness. 
in the second sentence. Furthermore, due to the design of the stimuli, the subjects were not able to develop a strategy of simply referencing the pronoun with the first person mentioned in the leading sentence. This is true since the first person mentioned in the leading sentence was the correct referent only half of the time.

The mean lengths of the second sentences of the sentence pairs (i.e. the verbconsistent and verb-inconsistent sentences) were matched in terms of the number of words. The mean lengths of these sentences were 9.05 and 9.0 , respectively. It was important that these sentences were matched in terms of length since it was the reaction time to these sentences that was the crucial dependent variable.

Twenty-eight additional trials were included, the purpose of which was to encourage subjects to read the leading sentence. On those trials, a leading sentence of a similar syntactic construction to that used in the experimental sentences was displayed followed by a sentence which was in the form of a question ${ }^{2}$. These questions were clearly indicated by a box surrounding them and subjects were simply required to answer the question. Note that the questions were offset from the experimental sentences in this way because the subject's task was slightly different when responding to the questions as compared to when responding to the experimental sentences (i.e. answering a question versus identifying a referent). It was, therefore, the intention that the box would alert the subject to this change in task. The data for these filler trials were not included in the analysis.

The sentences were divided into 4 blocks. Only one instance of each verb was represented in each block and at least one instance of each combination (e.g. CC-VC) was represented in each block. The sentences within each block were randomly ordered and the number of responses to the first person mentioned versus the second person mentioned was equally distributed across blocks. The order of presentation of blocks was counterbalanced across subjects. Six practice sentences, representing the types of sentences to be encountered, preceded the presentation of the experimental stimuli. 
The stimuli for this experiment, and for all following experiments reported, were presented in the center of an IBM computer screen using the Micro Experimental Laboratory Program (MEL) (Schneider, 1988). First the leading sentence appeared. It remained on the screen until the subject pressed a designated button on a response box that was interfaced with the computer, at which time it disappeared from the screen to be followed by either an experimental or filler sentence. Below both the experimental and filler sentences were the names of the two people mentioned in the sentence. Subjects responded by pressing one of two other buttons on the response box. The buttons corresponded to either the first or the second person mentioned in the sentence. All responses were made with a finger of the right hand. Once a response was made, the leading sentence of the next sentence pair would appear. The computer recorded both reaction time (with a time base of 0.001 seconds) and accuracy. Reading times for the leading sentences were recorded separately from the reaction times to the second sentences, but did not figure in the analyses.

Procedure. Subjects were tested individually. Each subject saw all the stimuli. Subjects were told that a sentence would appear in the center of the screen. They were instructed to read the sentence silently and to press a designated button on the response box when finished. They were further instructed that following the presentation of this sentence would be one of two possibilities; either a second sentence would follow that would be of the form "Anne defended Gail because she was innocent", or the form of the second sentence would be a question. In the former instance their task was to decide to whom the pronoun, "he" or "she" in the sentence referred. In the case of the questions, their task was to simply answer the question. The questions were always about one of the two people mentioned in the sentence (e.g. "Who was an experienced camper?"). In both cases, the subjects were to indicate their choice by pressing the appropriate button on the response box. Subjects were also told that at times there would not be a leading sentence, but rather a string of X's would appear. In such instances they were instructed to simply 
press the button on the response box that erases the leading sentence from the screen and continue with the task of deciding in the second sentence to whom the pronoun referred. Subjects were further instructed to read the sentences as a pair and to respond as quickly and accurately as possible.

Results

The experiment used a mixed design with one between groups factor and two within groups factors. The between groups factor was Group and it had two levels -- older adult (OA) and younger adult (YA). The two within groups factors were Context, with two levels -- context consistent (CC) and no context (NC) and Verb Consistency, also with two levels -- verb consistent (VC) and verb inconsistent (VI).

Only responses to the experimental sentences were analyzed. Separate analyses of variance with both subjects (F1) and items (F2) as random factors were performed on the mean reaction times for correct responses and on the proportion of errors. Min $\mathrm{F}^{\prime}$ values were also calculated and are reported only when significant. Extreme reaction time values within each condition (those less than or greater than the condition mean $\pm 3 \times$ standard deviation) were replaced by that value. The number of responses replaced was approximately the same for all conditions ranging from 1-2\% for both the YA and OA groups. Subjects of the OA group would occasionally press the wrong button on the response box and the response would not be registered by the computer. Such instances were recorded by the experimenter as mechanical errors and not included in the analyses. The number of mechanical errors was minimal and accounted for only $0.5 \%$ of the total number of possible responses.

Figure 5.1 shows subjects' reaction times to verb-consistent and verb-inconsistent sentences under the two context conditions for both groups. Analysis of the data by both subjects and items revealed significant main effects of Group $[F](1,38)=30.19, \mathbb{R}<0.001$; $\left.E_{2}(1,68)=1457.50, \mathbb{D}<0.001 ; \min \mathrm{F}^{\prime}(1,40)=29.6, \mathbb{p}<0.01\right]$, with the $\mathrm{OA}$ group slower than the YA group across all conditions, Context $[\mathrm{E} 1(1,38)=67.81, \mathrm{D}<0.001 ; \mathrm{E} 2(1,68)=$ 
$\left.61.31, \mathfrak{p}<0.001 ; \min \mathrm{F}^{\prime}(1,100)=32.2, \mathrm{~g}<0.01\right]$, with reaction times to $\mathrm{CC}$ sentences faster than to NC sentences for both groups, and Verb Consistency $[F 1(1,38)=38.15$, $\left.\mathrm{p}<0.001 ; \mathrm{E} 2(1,68)=14.83, \mathrm{p}<0.001 ; \min \mathrm{E}^{\prime}(1,103)=10.68, \mathrm{p}<0.01\right]$, with reaction times to VI sentences slower than to VC sentences for both groups. No interactions were significant by both subjects and items.

Figure 5.2 shows the accuracy data for both groups of subjects. The analysis revealed significant main effects, by both subjects and items, of Group $[E 1(1,38)=4.23$, $\mathfrak{p}<.05 ; \mathrm{F} 2(1,68)=22.60, \mathrm{p}<0.001]$, with overall more errors made by the OA group than by the YA group, Context $[\mathrm{E} 1(1,38)=7.16, \mathrm{p}<0.05 ; \mathrm{E} 2(1,68)=3.94, \mathfrak{p}=0.05]$, with more errors made on NC than CC sentences, and Verb Consistency $[\mathrm{F} 1(1,38)=15.97$, $\left.\mathrm{p}<0.001 ; \mathrm{E2}(1,68)=8.57, \mathrm{p}<0.01 ; \min \mathrm{F}^{\prime}(1,106)=5.58, \mathfrak{p}<0.05\right]$, with more errors made on VI than VC sentences. In contrast to the reaction time data, the interaction of Context $x$ Verb Consistency was also significant by both subjects and items $[E 1(1,38)=$ $8.25, \mathfrak{p}<0.01 ; \mathrm{E2}(1,68)=4.85, \mathfrak{p}<0.05]$. Tests of simple main effects revealed that there was a significant effect of verb-consistency at the level of $\mathrm{NC}[\mathrm{E}](1,38)=18.50, \mathfrak{p}<0.001$; $\left.\mathrm{E} 2(1,68)=13.15, \mathrm{p}<0.001 ; \min \mathrm{F}^{\prime}(1,105)=7.69, \mathrm{p}<0.05\right]$ but not at the level of $\mathrm{CC}$ $[F 1(1,38)=.89]$. In other words, for both groups of subjects, more errors were made on VI sentences than on VC sentences when there was no preceding context. However, there was no significant difference between the number of errors made on the these types of sentences when there was a preceding context ${ }^{3}$.

A supplementary analysis was conducted to investigate whether the type of verb (NP1 versus NP2) interacted with any of the other factors (i.e. group, context, verb consistency). Since the pronoun in the subordinate clause occupied the subject position, it was hypothesized that if the effect of verb consistency held only for NP1 verbs, then one could argue that a parallel function strategy (Sheldon, 1974) was being used by the subjects and not the implicit causality of the verbs. However, this hypothesis was not supported. For the reaction time data none of the interactions involving this factor was significant by 
Figure 5.1: Mean RT to VC vs. VI sentences by context
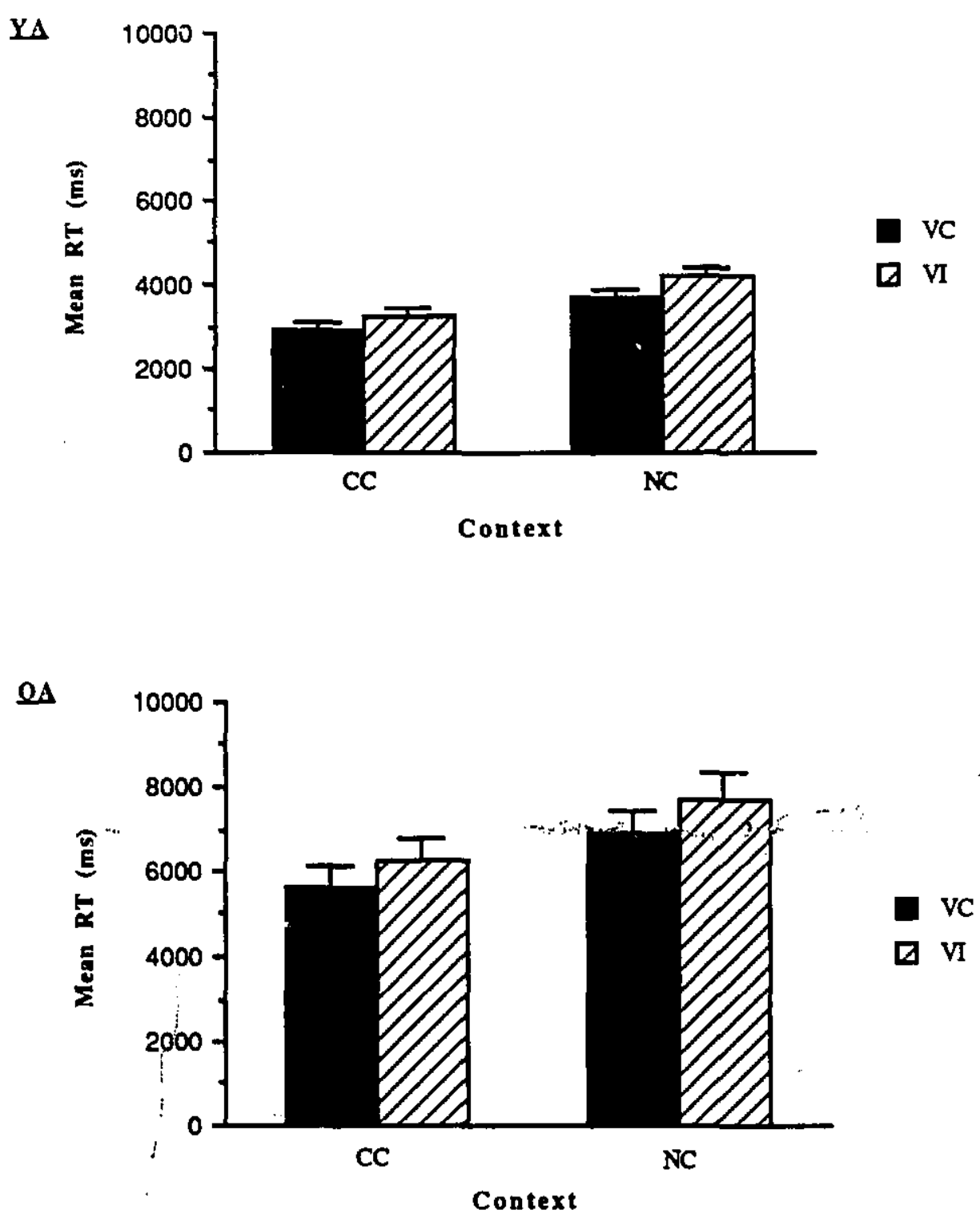
Figure 5.2: Mean \% errors to VC vs. VI sentences by context YA
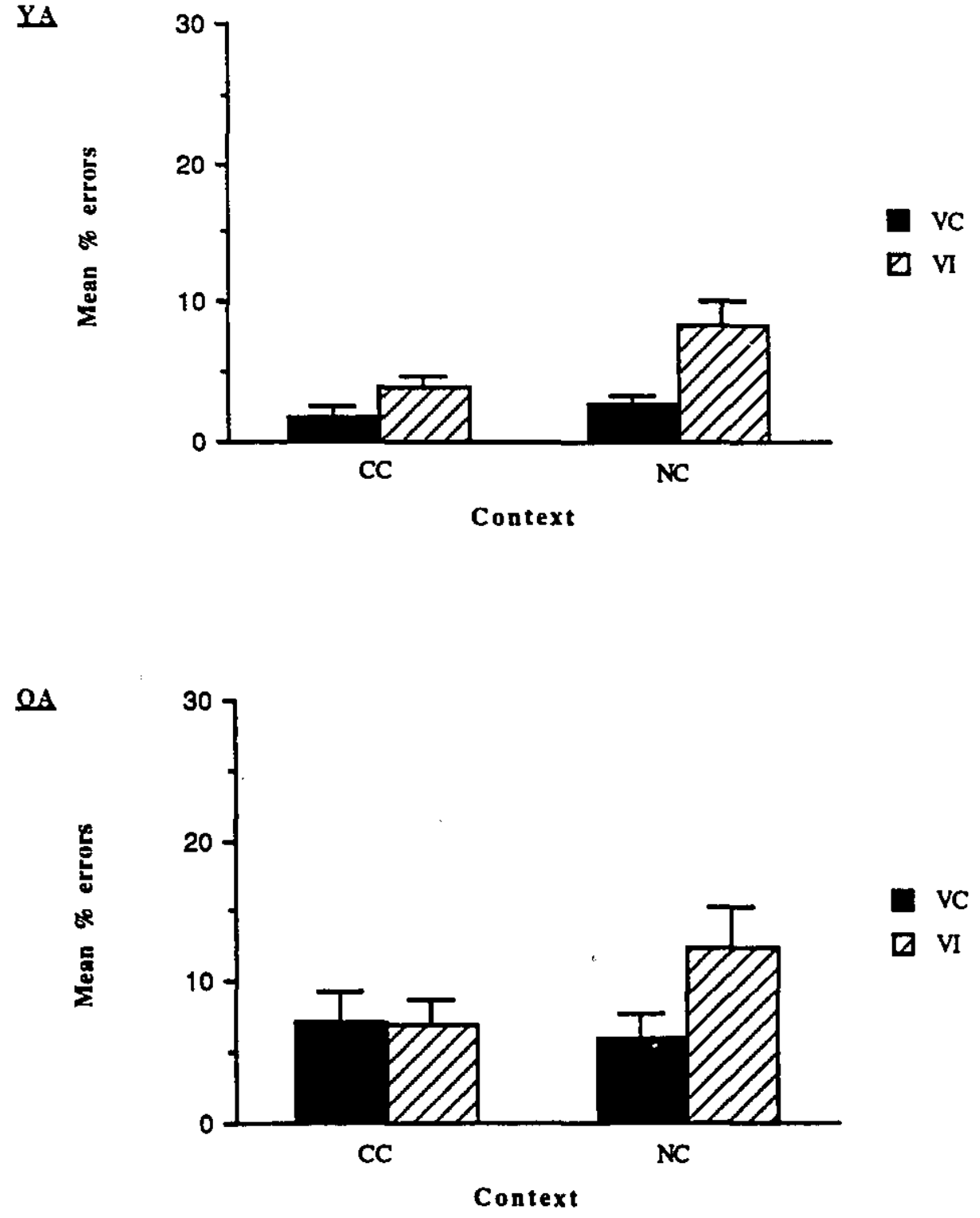
both subjects and items (Verb Type $x$ Verb Consistency $[E 1(1,38)=29.37, \mathbb{R}<0.001]$; Group $x$ Verb Type $x$ Verb Consistency $[E 1(1,38)=4.12, \mathbb{R}<0.05])$. For the accuracy data there were no interactions involving the factor Verb Type.

Discussion

The results of this experiment provide evidence for the use of contextual information in resolving pronouns. Furthermore, these results are in agreement with the current view that older adults are capable of using contextual information and making inferences for language processing (Belmore, 1981; Burke \& Yee, 1984; Light et al., 1982; Light \& Albertson, 1988; Light and Capps, 1986; Till, 1985; Zacks \& Hasher, 1988; Zelinski \& Miura, 1990) when the demands placed upon working memory are minimal. Although, overall, the $\mathrm{OA}$ group responded more slowly and made more errors than did the YA group, they demonstrated exactly the same pattern as the YA group.

The fact that reaction times were faster to sentences preceded by a leading sentence than to those that were not preceded by one argues for the notion that the information contained in this preceding context was used in resolving the pronoun. Of particular interest was the finding that while an effect of context was present, it was not sufficient to override the effects of the bias of the verbs, in terms of processing time. Regardless of the context condition, reaction times to verb-inconsistent sentences were significantly slower than to verb-consistent sentences. Interestingly, support for the hypothesis that contextual information can modulate the implicit causality of the verb was suggested by the accuracy data. For both groups of subjects, the effect of implicit causality found in the no context condition was nullified by the presence of a leading sentence. That is, under the context consistent condition, there was no significant difference in the number of errors made on verb-consistent and verb-inconsistent sentences. However, it is important to acknowledge that there is no evidence to support the notion that contextual information was necessarily used prior to the initial coindexation of the pronoun. It is equally likely that it was used in 
the recovery of the misassignment. Therefore, the initial assignment could still have been influenced by the implicit causality of the verb.

With reference to the emergence of a context effect in reaction times, one might argue that the faster reaction times under the context consistent condition were simply the result of activating potential referents prior to the task of having to select a referent. In other words, by having the referent names already activated in working memory, it is possible that the processing time required to select a referent when the situation arose was simply reduced. Under this account, the actual semantic information contained in the leading sentence did not serve to aid in the resolution of the pronoun.

Pilot Study $2 \mathrm{~b}$ attempted to distinguish between these possibilities by adding $\mathrm{a}$ third context condition in which the information contained in the leading sentence was unrelated to the reason provided for the action or emotion in the second sentence. If, in fact, reaction times were faster with a preceding context simply because of the activation of the referents then it was hypothesized that reaction times to sentences under this third condition should also be faster than to those sentences not preceded by a context. 


\section{Pilot Study 2b}

Method

Subjects. Two groups of subjects were tested. The Younger Adult (YA) group consisted of 20 university students ranging in age from 19 years to 30 years (mean age = 23 years). The Older Adult (OA) group consisted of 15 elderly individuals, all with secondary school education and nine with post-secondary experience, ranging in age from 52 years to 71 years (mean age $=63$ years). All subjects were right-handed and their native language was English. None of the subjects participating in this study had participated in any of the previous pilot studies.

Materials. Apparatus and Procedure. The stimuli used in this experiment were similar to those used in the first experiment. With respect to the factor Verb-Consistency, the VC and VI sentences remained the same ${ }^{4}$. With respect to the factor Context, the levels $\mathrm{NC}$ and $\mathrm{CC}$ remained the same, but sentences under $\mathrm{CC}$ were changed slightly in that the amount of information they provided was reduced to one clause. However, the major difference with respect to this factor was the addition of a third level. This third level was termed context unrelated (CU). Under this condition, the information contained in the leading sentence was unrelated to the reason provided in the second sentence for the action or emotion expressed in that sentence. The resulting combination of sentence pairs was as shown in Table 5.2 (see Appendix B for a complete listing of the stimuli).

As in the Pilot Study $2 a$, filler sentences $(n=30)$ that were of the same syntactic constructions as the experimental sentences were used to ensure that the subjects were reading the leading sentences for meaning.

The sentences were divided into six blocks with only one instance of each verb represented in each block. The sentences within each block were randomly ordered. Three orders of presentation of the blocks were used and the order of presentation of blocks was counterbalanced across subjects. Eight practice sentences, representing the types of 
Table 5.2

Examples of Stimuli for Pilot Study $2 b$

Note that the verb used in these stimuli, confided, is a NP1 type verb.

CC-VC Jane had a serious problem Gail discovered. Jane confided in Gail because she needed advice.

CC-VI Jane believed that Gail was good at counselling. Jane confided in Gail because she could offer advice.

CU-VC Jane was bored with the movie Gail thought. Jane confided in Gail because she needed advice.

CU-VI Jane knew that Gail liked the colour red. Jane confided in Gail because she could offer advice.

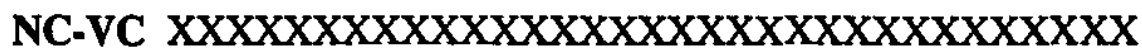
Jane confided in Gail because she needed advice.

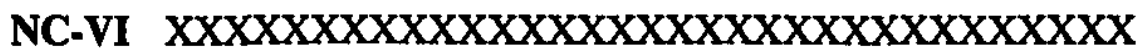
Jane confided in Gail because she offered advice. 
sentences to be encountered, preceded the presentation of the experimental stimuli. The procedure used was identical to that used in the Pilot Study 2a.

Results

As in Pilot Study 2a, this experiment used a mixed design with one between groups factor and two within groups factors. The between groups factor was Group and it had two levels -- older adult (OA) and younger adult (YA). The two within groups factors were Context, this time with three levels -- context consistent (CC), no context (NC) and context unrelated (CU) and Verb Consistency, with two levels -- verb consistent (VC) and verb inconsistent (VI).

Analysis of the data proceeded in the same manner as in Pilot Study 2a. Again, extreme reaction time values within each condition (those less than or greater than the condition mean $\pm 3 \times$ standard deviation) were replaced by that value. The number of responses replaced was approximately the same for all conditions ranging from 0.5 to $2.0 \%$ for the YA and 0.3 to $2.0 \%$ for the OA groups. Mechanical errors only occurred for the OA group. The number of mechanical errors was low and accounted for only $1.7 \%$ of the total number of possible responses. It is of interest to note that of this $1.7 \%, 1.4 \%$ was made under the no context condition.

Figure 5.3 shows subjects' mean reaction times to verb-consistent and verbinconsistent sentences under the three context conditions for both groups. Analysis of the data by both subjects and items revealed significant main effects of Group $[E 1(1,33)=$ 5.73, $\left.\mathrm{p}<.05 ; \mathrm{E} 2(1,102)=610.56, \mathrm{p}<0.001 ; \min \mathrm{F}^{\prime}(1,34)=5.68, \mathrm{p}<0.05\right]$, with the $\mathrm{OA}$ group slower than the YA group across all conditions, Verb Consistency $[\mathrm{E} 1(1,33)=$ 51.47, $\left.\mathrm{D}<0.001 ; \mathrm{E} 2(1,102)=19.32, \mathrm{p}<0.001 ; \min \mathrm{E}^{\prime}(1,134)=14.05, \mathrm{p}<0.01\right]$, with reaction times to VI sentences slower than to VC sentences for both groups across all context conditions, and Context $[\mathrm{E} 1(2,66)=4.22, \mathrm{p}<.05 ; \mathrm{E} 2(2,102)=3.14, \mathrm{p}<.05]$. Pairwise comparisons of the means under this condition using the Newman-Keuls procedure indicated that reaction times to sentences under CC $($ mean $=4519 \mathrm{~ms})$ were 
Figure 5.3: Mean RT to VC vs. VI sentences by context
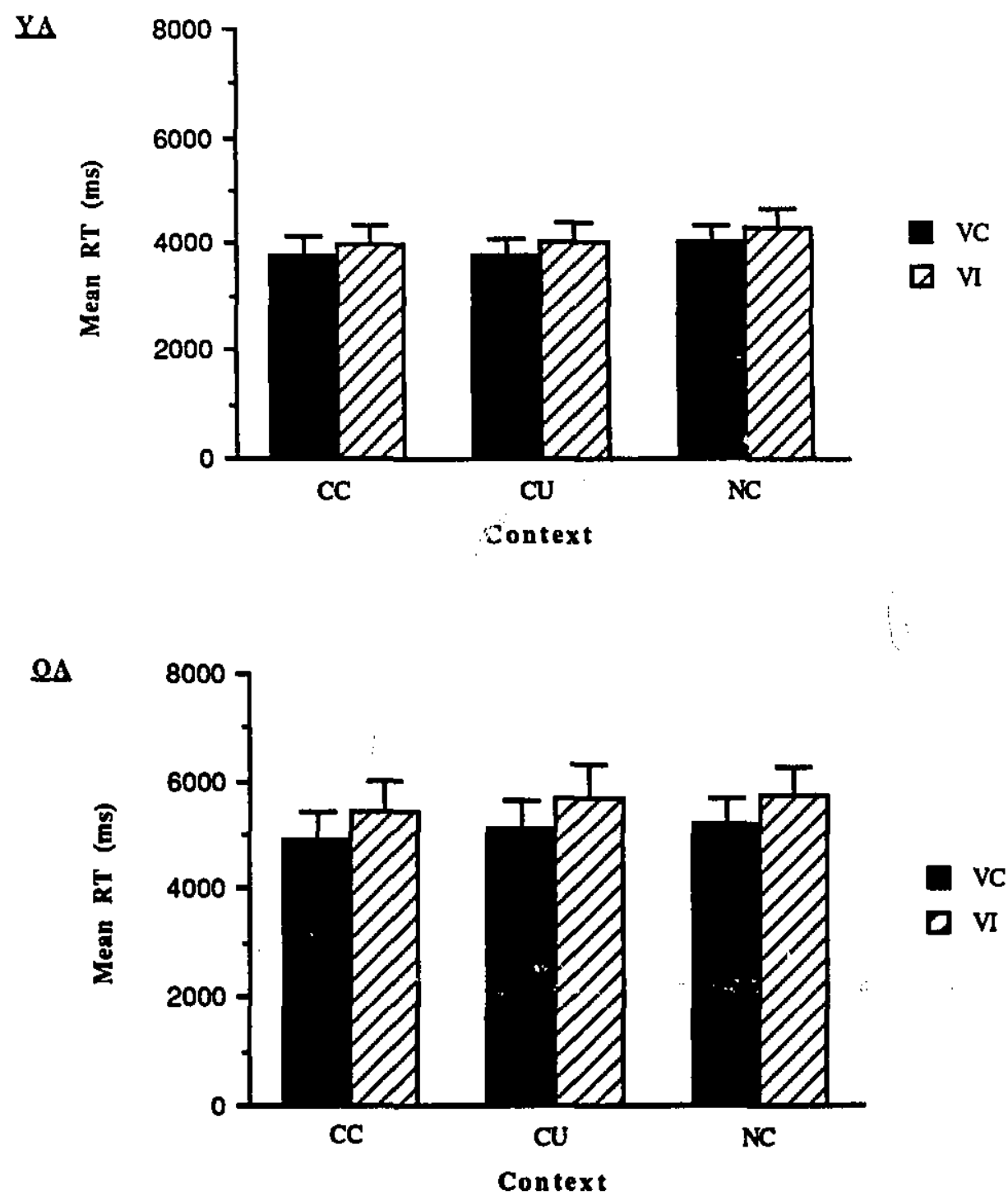
Figure 5.4: Mean \% errors to VC vs. VI sentences by context
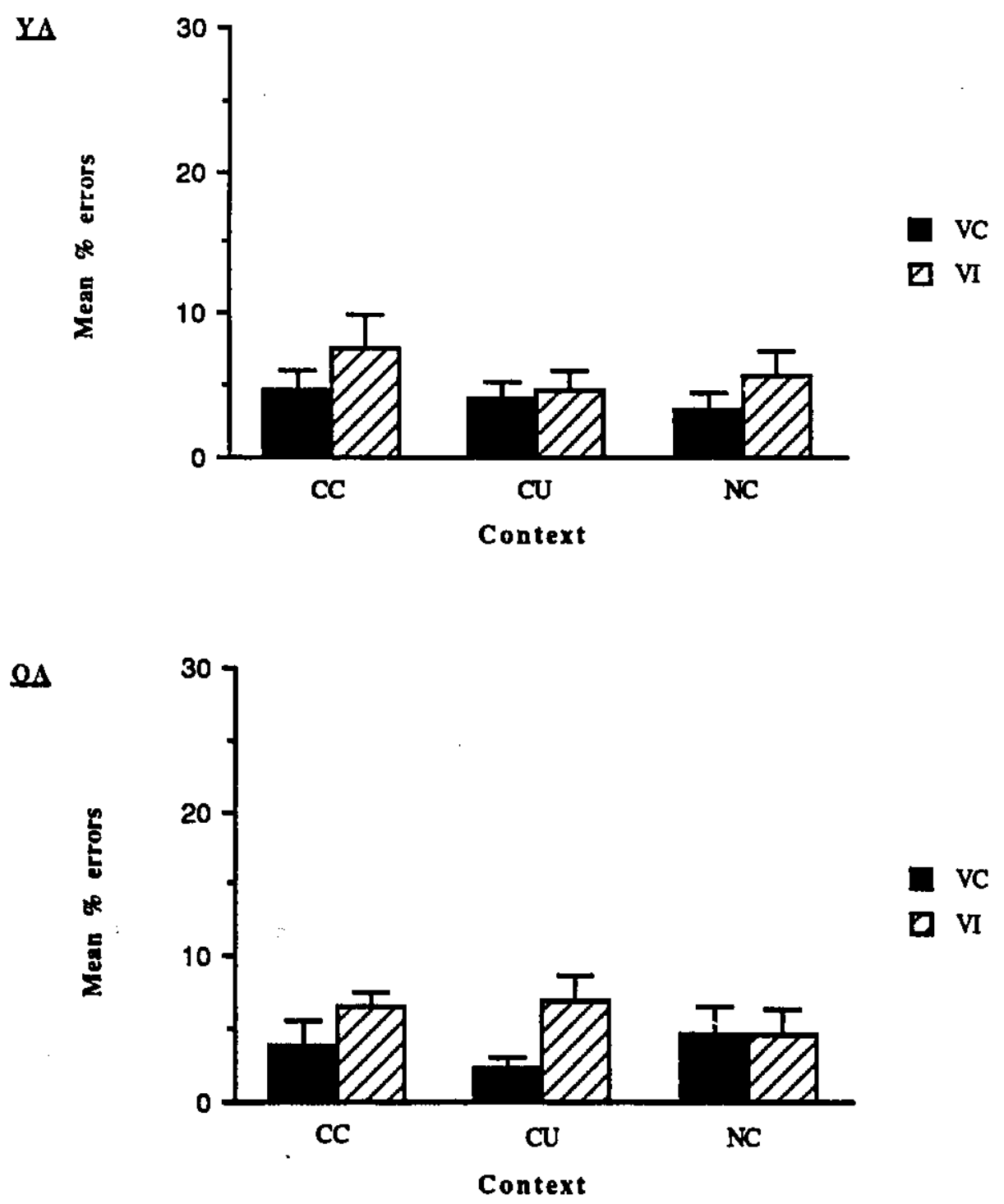
significantly faster than to those under $N C$ (mean $=4820 \mathrm{~ms})(p=.05)$, by both subjects and items. Reaction times to sentences under the CU condition (mean $=4660 \mathrm{~ms}$ ) were not significantly different than to those under either the $\mathrm{NC}$ or $\mathrm{CC}$ conditions by either subjects or items. The only significant interaction found was Group x Verb Consistency $[E](1,33)$ $=6.14, \mathfrak{R}<.05 ; \mathrm{E} 2(1,102)=6.33, \mathrm{p}<.05]$. Tests of simple main effects revealed an effect of Verb Consistency at both levels of Group -- YA $[\mathrm{Fl}(1,33)=4.29, \mathrm{R}<0.05 ; \mathrm{E} 2(1,164)=$ $6.45, \mathrm{R}<.01]$ and $\mathrm{OA}\left[\mathrm{El}(1,33)=13.59, \mathrm{R}<0.001 ; \mathrm{E2}(1,164)=25.65, \mathrm{R}<0.001 ; \min \mathrm{E}^{\prime}\right.$ $(1,73)=8.88, \mathrm{p}<0.01]$. The interaction probably reflects the fact that there is a larger and more robust difference (as indicated by a significant $\min \mathrm{F}^{\prime}$ ) between reaction times to $\mathrm{VC}$ and VI sentences for the OA group than for the YA group.

Figure 5.4 shows the accuracy data for both groups of subjects. Only a significant main effect of Verb Consistency $[\mathrm{E} 1(1,33)=9.9, \mathrm{p}<0.01 ; \mathrm{E} 2(1,102)=5.01, \mathrm{p}<0.05]$ was found. For both groups, more errors were made on VI sentences than or VC sentences 5 . Discussion

Overall, these results confirm those of Pilot Study $2 \mathrm{a}$ and provide evidence for the notion that contextual information is used in the resolution of pronouns. Furthermore, these results suggest that this information is used equally well by older and younger adults. When the leading sentence provided information that was consistent with the reason provided in the second sentence, reaction times to both verb-consistent and verbinconsistent sentences were faster than when there was no context present. Of particular interest was the finding that responses to verb-consistent and verb-inconsistent sentences under the context unrelated condition were not significantly faster than responses to those sentences under the no context condition. This finding may be viewed as support for the proposal that the faster reaction times under the $\mathrm{CC}$ condition are a reflection of the use and integration of the semantic information in the leading sentence to ultimately find the correct referent of the pronoun and not simply a result of the activation of the referents. As discussed earlier, if the faster reaction times were simply a matter of activating referents, 
then reaction times under the $\mathrm{CU}$ condition should have also been significantly faster than those under the NC condition. However, this claim is somewhat weakened by the finding that reaction times to sentences under the $\mathrm{CC}$ condition were not significantly faster than to those under the CU condition. This result suggests that there may be at least some benefit, in terms of reduced processing time, provided by a context which does not necessarily support the interpretation of a referent semantically, but which nonetheless establishes it in a discourse model and, therefore, makes it more readily accessible.

Consistent with the results of Pilot Study 2a, the reaction time data failed once again to provide support for the notion that contextual information can modulate the effect of the bias created by the implicit causality of the verb. Reaction times to verb-inconsistent sentences were slower than reaction times to verb-consistent sentences across all context conditions. This finding was also reflected in the accuracy data, wherein overall more errors were made on verb-inconsistent sentences than on verb-consistent sentences. Notice that this finding with respect to the accuracy data is contrary to the results of Experiment $2 \mathrm{a}$ in which there was no difference in the number of errors to VC and VI sentences under the $\mathrm{CC}$ condition. Upon closer inspection of the accuracy data for Pilot Study $2 \mathrm{~b}$, however, it becomes clear that, although the three-way interaction between age, context, and verb consistency was not statistically significant, it is not the case that more errors were made on VI than VC sentences under all context conditions. Most notably, for the older adults the number of errors made to VC and VI sentences is approximately the same under the no context condition. This is surprising and difficult to explain. Such discrepant results, however, force us to interpret the results of the accuracy data in Pilot Study 2a with reservation.

\section{General Discussion for Pilot Studies 2a and $2 \mathrm{~b}$}

This investigation was undertaken to investigate the possible influence of contextual information on the resolution of pronouns. Of particular interest was the question of whether or not the use of contextual information could serve to modulate the effect of the 
implicit causality of verbs. Of secondary interest was the possibility of age differences with respect to the use of this information.

First, it should be noted that no relevant age differences were found in any of the experiments, in terms of the use of contextual information. Older adults were found to use the contextual information found in the leading sentence as well as younger adults. Further evidence for their use of this information was found in their sensitivity to the verb consistency factor, resulting in an effect of implicit causality. These results suggesting no age differences in the use of contextual information are consistent with a number of studies (Cohen \& Faulkner, 1983; 1984; Light \& Capps, 1986; Madden, 1988) and with the emerging view that the ability to use this information and make inferences is preserved in old age when the demands placed upon working memory are minimal (Burke \& Yee, 1984; Light et al., 1982, Light \& Albertson, 1988; Light, Valencia-Laver, \& Zavis, 1991; Zelinski \& Miura, 1990). The following discussion is, therefore, equally applicable to both age groups.

Both Pilot Studies $2 \mathrm{a}$ and $2 \mathrm{~b}$ found that reaction times for specifying the correct referent of a pronoun are faster to sentences that are preceded by a leading sentence whose information is consistent with the information provided in the subordinate clause of the second sentence than to those that are not preceded by a leading sentence. These findings may be interpreted as support for the notion that contextual information is used to aid in the resolution process. Note that the pronoun could have been resolved based solely on information present in the second sentence (as must necessarily be done under the NC condition). Therefore, it can be argued that the preceding sentence served to set up a discourse model which, in turn, provided a context in which to interpret the subsequent sentence, yielding faster reaction times. Such a view is consistent with that of Bosch (1983) and Brown and Yule (1983). Recall that they both view the participants of a discourse as continually developing models as the discourse evolves, that serve to represent the environment in focus. Accordingly, they argue that speakers, when using an anaphor, 
will do so upon considering the hearer's discourse model and will choose an intended referent that is believed to be within the hearer's model. Similarly, it is believed that hearers (or readers) will also be operating with this strategy in mind, and resolve the anaphor based on their present discourse model. Granted, in these experiments the discourse model was limited and based only on one previous utterance, but apparently it was still influential, in terms of processing time, in the resolution of the pronoun. However, the question concerning whether the information in the leading sentence made the referents more readily accessible as a function of simply establishing them in the discourse model or whether the semantic information was influential in highlighting a preferred referent was not answered adequately by Pilot Study $2 b$. The finding that reaction times to sentences under the $\mathrm{CU}$ condition were not significantly different than to those under either the $\mathrm{NC}$ or $\mathrm{CC}$ conditions, leaves the question concerning the effect of unrelated contextual information on pronoun resolution unresolved.

The hypothesis that contextual information could serve to modulate the effect of the implicit causality of the verb was not strongly supported. In terms of reaction time data, the results of both Pilot Studies $2 \mathrm{a}$ and $2 \mathrm{~b}$ indicated that the difference between verb consistent and verb inconsistent sentences present under the no context condition remained under the context consistent condition. In terms of the accuracy data, there was some indication that the contextual information could serve to reduce the number of errors to VI sentences. However, there was a discrepancy of results between Pilot Studies 2a and 2b. In Pilot Study 2a the leading sentence served to eliminate the difference between the proportion of errors made on the verb consistent and verb inconsistent sentences that was found under the no context condition. This result, however, was not supported in Pilot Stucily $2 b$, wherein the difference between the proportion of errors made on VC and VI sentences remained under the $\mathrm{CC}$ condition. Moreover, closer inspection of the data from Pilot Study $2 b$ revealed that for the older adults, under the no context condition, there was not the expected effect of implicit causality. These discrepant results force us to question 
the reliability of the error data in Pilot Study 2a and, therefore, to interpret it with reservation. Furthermore, as explained in the discussion of Pilot Study 2a, the effect of implicit causality may still have persisted under the $\mathrm{CC}$ condition with the contextual information influencing only the recovery of an initial misassignment.

This possibility forces us to address the issue of modularity of the language system and the extent to which the results of this investigation speak to this issue. The key question with respect to these results concerns the point at which contextual information influences the resolution of pronouns. Is it influential at the point of initiation of coindexation or rather at some point further downstream in the process? Obviously, the off-line nature of the task does not allow us to address this point directly. However the results are suggestive of a certain position. Specifically, the results suggest that the contextual information is used following the initiation of the coindexation of the pronoun during the integration of the interpretation of the pronoun with the discourse. It is proposed that support for this position is found in the fact that reaction times to verb inconsistent sentences remained longer than to verb consistent sentences even when preceded by a supporting context. Since context was not sufficient to nullify the effects of the implicit causality of the verbs in terms of processing time, one can be fairly confident that it was not influential during the initial coindexation of the pronoun. The bias of the verb initially favored a particular referent, and it was only after committing to this initial preference that the final interpretation of the pronoun was influenced by the context. It should be noted that one cannot discount the possibility that both the context and the implicit causality of the verb were used in the initial coindexation of the pronoun. Under this account, one could argue that CC-VC was still faster than CC-VI simply because in the former condition both the context and the verb bias support the same referent; whereas under CC-VI, the context and the implicit causality of the verb support different referents. The fact remains, however, that under the CC-VI condition, upon encountering the pronoun, the reader must have committed to the reading imputed by the implicit causality of 
the verb or else there would have been no reaction to the inconsistency of the information in the second clause of the sentence to the verb's bias. Therefore, it still seems most likely that the contextual information was influential in the resolution of the pronoun following the initial coindexation.

The view that contextual information is influential in pronoun resolution only following initial coindexation of the pronoun and its referent is consistent with the view of a modular language system (Fodor, 1983; Forster, 1979). As discussed earlier, Fodor acknowledges the possible influence of contextual information on language processing by invoking the notion of a "context analyzer". The context analyzer is believed to act upon information provided by the parser concerning the line of analysis it is taking and determine whether or not a particular analysis is consistent with the facts provided by the context. Note, however, that the context analyzer cannot direct a particular line of analysis. In this sense, the notion of modularity is maintained in that contextual information does not guide parsing decisions. With respect to the results of this investigation it may be argued that the initial coindexation was a function of the implicit causality of the verb, uninfluenced by context, but that the contextual information was used in supporting a particular analysis, reflected in faster reaction times to context consistent sentences than to no context sentences, and possibly in promoting a better recovery of a misanalysis as suggested by the error data of Pilot Study 2a. It must be re-emphasized that the results of these two studies do not directly speak to this issue and, therefore, this discussion is purely speculative.

It is of interest to note that the finding that the contextual information could not modulate the influence of the implicit causality of the verb is consistent with the view of Shapiro, Zurif and Grimshaw (1987; 1989). They have found evidence to suggest a "contextual impenetrability" of verbs. In a series of studies they demonstrated that a verb's representational complexity, as defined by the number of possible argument structures a verb may realize (as opposed to the number of syntactic subcategorizations) affects sentence processing. In a cross-modal lexical decision task, reaction times were 
significantly faster when sentences contained verbs with fewer potential argument structures. Of particular interest was the finding that this effect of verb complexity was not influenced by a preceding context that surongly biased a particular argument structure.

These results suggested that during processing, all of a verb's potential argument structures are temporarily activated, irrespective of the information contained in the preceding context. Shapiro et al. (1989) interpreted this finding to suggest that "verb processing in sentences involves a contextually impenetrable subcomponent of the language comprehension system" (p.242). The results of the present investigation further support this position by suggesting that other semantic properties of verbs, such as implicit causality, are also resistant to contextual forces.

To conclude, Pilot Studies $2 \mathrm{a}$ and $2 \mathrm{~b}$ have found evidence to suggest that contextual information is influential in the resolution of pronouns by both younger and older adults. It has been suggested that this information is used to create discourse models in which to interpret subsequent pronouns. This account is consistent with the view of anaphor resolution proposed by Bosch (1983) and Brown and Yule (1883). It has been argued that contextual information is not used to guide initial coindexation decisions, but rather during subsequent integration processes, in line with a modular view of language processing. This view is supported by the fact that the strength of the effect of contextual information is not sufficient to override the effect of the implicit causality of verbs -- thereby, supporting the notion of the contextual impenetrability of verbs proposed by Shapiro et al. (1987; 1989). It is important to stress that the interpretation of these results supports the view of pronoun resolution in which coindexation is initiated immediately as relevant cues become available (e.g. the implicit causality of verbs), but that final interpretation is subject to contextual influences (e.g. Sanford \& Garrod, 1989; Vonk, 1985b). 


\section{Chapter 6: Pilot Study 3}

The result of Pilot studies 1, 2a and $2 \mathrm{~b}$ were important in establishing: 1) that older adults respond to the implicit causality of verbs in the same manner as do younger adults and; 2) that both older and younger adults are influenced by contextual information in the resolution of pronouns. As discussed earlier, an interesting question with respect to the influence of contextual information on language processing by RBD patients involves whether there is a difference between the ability to use information specifically stated in the text versus the ability to use contextual information based on general world knowledge. Thus, the purpose of Pilot Study 3 was to lay the foundation for such an inquiry by first investigating the abilities of older adults to use general world knowledge to pragmatically constrain antecedent choices. As well, this experiment was to serve as a replication of Hirst and Brill (1980) and confirm the notion that contextual information is important in pragmatically constraining antecedent choices for pronouns by younger adults. Recall that Hirst and Brill found that reaction times to sentences that biased a preferred antecedent for an ambiguous pronoun based on pragmatic constraints were faster than to more neutral sentences (e.g. John stood watching while Henry fell down some stairs. He ran for a doctor/He thought of the future).

The results of studies investigating the abilities of older adults to resolve pronouns have been mixed. There is some evidence to suggest that older and younger adults do not differ in their abilities to use contextual information to resolve pronouns when the demands placed upon working memory are minimal (LeDoux, Blum \& Hirst, 1983; Light and Capps, 1986). For example, using a subset of the items used in Hirst and Brill (1980), LeDoux et al. (1983) found that OA and YA did not differ on their choice of a preferred referent that was constrained by pragmatic considerations based on the use of contextual information. Similarly, Light and Capps (1986), also using materials adapted from Hirst and Brill (1980), found that older adults were as capable as younger adults of selecting preferred referents based on a biasing context when the demands placed upon working 
memory were minimal (i.e. when the pmnominal sentence immediately followed the leading sentence). Key to the issue of the influence of working menory demands, however, was the finding that when material was interjected between the leading and pronominal sentences, older adults demonstrated less of a tendency to choose the preferred referent than did younger adults. These results suggested that as long as working memory demands were minimal there was no difference between OA and YA in their abilities to use contextual information to resolve pronouns; however, when working memory was sufficiently taxed, the ability of older adults to use contextual information to resolve pronouns was impaired.

In contrast to these findings suggesting that age differences in the abilities of older and younger adults to resolve pronouns are a function of working memory capacity, Light and Anderson (1985) found age related deficits in the ability to identify the referents of pronouns that did not appear to be simply a function of reduced working memory capacity. Subjects were presented with paragraphs and required to read the paragraphs at their own pace. After reading the paragraphs they were asked two questions, one of which queried the referent of a pronoun that was introduced in the final sentence of the paragraph. The distance of the antecedent from the pronoun was varied. The authors hypothesized that if age differences in pronoun resolution only resulted as a function of working memory capacities, then age differences should emerge only when the antecedent and the pronoun were at greater distances from one another. However, contrary to this expectation, older adults were significantly poorer than younger adults at identifying the referent of a pronoun at all distances of the antecedent. Light and Anderson (1985) could offer no explanation for this discrepancy between the studies. However, upon consideration of the stimuli, there appear to be two factors in particular that may have influenced the results. Unfortunately, the authors do not give examples of the stimuli they used, but in their description of them there are two points to note. The first is that the paragraphs themselves were relatively long, consisting of twelve sentences, presented one sentence at a time. It 
seems that even if the correct antecedent had been in the sentence immediately prior to the pronominal sentence, thereby reducing working memory demands according to Light and Anderson (1985), simply having to process the whole paragraph was likely to tax working memory at some level. Therefore, age differences found at this position may still have been a function of reduced working memory capacity for the OA. An additional confound concems the fact that, on average, in each individual paragraph, six possible antecedents were introduced. In contrast, in the stimuli used by Light and Capps (1986), there were always only two possible antecedents. The intervening sentences they used did not introduce any new characters. The addition of possible referents in the stimuli used by Light and Anderson (1985) necessarily placed more demands on working memory and, therefore, made their stimuli more difficult to process, even when the correct referent was only in the sentence immediately preceding the pronominal sentence. Given these considerations, it is not completely surprising that the OA group in the Light and Anderson (1985) study still had more difficulty resolving pronouns at apparently small pronounantecedent distances. The nature of the stimuli used most likely placed a burden on working memory capacities regardless of the position of the antecedent. Therefore, it is possible that the age differences found by Light and Anderson (1985) may still be attributable to reduced working memory capacity.

However, it should also be noted that the results of Pilot Study 2a suggested that while older adults are able to use contextual information to resolve pronouns in a similar manner to younger adults, in terms of demonstrating an effect of context based on reaction times, they nonetheless tended to make more errors than did younger adults. Moreover, given that the disambiguating information was present in the second sentence, one cannot simply attribute reduced performance by the older adults to working memory impairments.

In view of these mixed results, the present study was conducted with a primary purpose of shedding further light upon the issue of the ability of older adults to use contextual information to resolve pronouns. As in LeDoux et al. (1983) and Light and 
Capps (1986), the stimuli used in this study were based on those used in Hirst and Brill (1980). However, the methodology used in this study differed in important ways. First, unlike in LeDoux et al. (1983), the leading sentence disappeared from view prior to viewing the pronominal sentence. As well, in this study both speed and accuracy were stressed as opposed to just accuracy. In contrast to Light and Capps (1986), a number of differences emerge. First, all of the stimuli used in Hirst and Brill (1980) were used in this experiment (details of which will be elaborated upon in the Materials section). Also, the stimuli were presented visually (as opposed to auditorily) and the method of response was identical to that used by Hirst and Brill (1980). Finally, reaction times as well as frequency of choice of preferred referents served as the dependent variables.

Method

Subjects. Two groups of subjects were tested. The Younger Adult (YA) group consisted of 20 university students ranging in age from 20 years to 30 years (mean age = 23 years). The Older Adult (OA) group consisted of 20 elderly individuals ranging in age from 58 to 72 (mean age $=67$ years). The mean years of education was 16.4 and 14.4. for the YA and OA groups, respectively. All subjects were right-handed and their native language was English.

Materials and Apparatus. The stimuli were identical to those used by Hirst and Brill (1980)6. They consisted of pairs of sentences of the form John (Henry) Xed while Henry (John) Yed. He Zed. (e.g. John stood watching while Henry fell down some stairs. He ran for a doctor. The variable manipulated in the construction of the stimuli was the plausibility of action $\mathrm{Z}$ in the pronominal sentence, given action $\mathrm{X}$ or $\mathrm{Y}$. Note that the assessment of plausibility depended upon the use of general world knowledge. For instance, in the example provided, it is more likely that someone "standing and watching" is in a position to "run" than someone who has "fallen down some stairs". The plausibility of an action given a particular situation determined the preferred referent of "he" in the second sentence. 
Plausibility ratings of sentence pairs were established by Hirst and Brill (1980) in a preliminary experiment. The resulting variable, termed Plausibility Difference, had 5 levels: 1) large positive, wherein the preferred referent was clearly the second NP ; 2) small positive, wherein the preferred referent was most likely the second NP; 3) large negative, wherein the preferred referent was clearly the first NP; 4) small negative, wherein the preferred referent was most likely the first NP; 5) neutral, wherein there was no obvious preferred referent. For the purpose of clarity, this factor and its levels were renamed for this investigation. The renamed factor was called Preferred Referent and the newly labeled levels were as follows: 1) NP1 Strongly Preferred (NP1-SP), wherein the preferred referent was clearly the first NP; 2) NP1 Likely Preferred (NP1-LP), wherein the preferred referent was most likely the first NP; 3) NP2 Strongly Preferred (NP2- SP), wherein the preferred referent was clearly the second NP; 4) NP2 Likely Preferred (NP2LP), wherein the preferred referent was most likely the second NP. The label of neutral (N), for which there was no preferred referent, remained as in Hirst and Brill (1980). The stimuli were constructed such that both John and Henry (who were the only referent names used in all of the stimuli) were the preferred referents an equal number of times and both appeared an equal number of times in the first and second position of the leading sentence. A total of 40 sentences was used. See Table 6.1 for examples of each type of sentence and Appendix $\mathrm{C}$ for a complete listing of the stimuli.

The sentences were divided into 2 blocks. The sentences within each block were randomly ordered with the restriction that identical leading sentences be separated by at least two other sentences. The order of presentation of the blocks was counterbalanced across subjects. Four practice sentences preceded the presentation of the experimental stimuli.

Both the presentation and response modes were identical to those used by Hirst and Brill (1980). The stimuli were presented in the center of a computer screen. First the leading sentence appeared. It remained on the screen until the subject pressed a designated 
Table 6.1

Examples of Stimuli for Pilot Study 3

NP1-SP Henry spoke at a meeting while John drove to the beach. He lectured on the administration.

NP1-LP Henry spoke at a meeting while John drove to the beach. He knocked over the water.

NP2-SP Henry spoke at a meeting while John drove to the beach. He brought along a surfboard.

NP2-LP Henry spoke at a meeting while John drove to the beach. He stopped at a store.

N Henry spoke at a meeting while John drove to the beach. He looked toward a friend. 
button on a response box that was interfaced with the computer, at which time the leading sentence disappeared and the pronominal sentence was displayed. Subjects responded by pressing one of two other buttons on the response box. These buttons corresponded to either "John" or "Henry". All responses were made with one finger of the right hand. Once a response was made, the leading sentence of the next sentence pair appeared. The computer recorded both reaction time (with a time base of 0.001 seconds) and accuracy 7 . Reading times for the leading sentences were recorded separately from the reaction times to the pronominal sentences, but did not figure into the analyses.

Procedure. Subjects were tested individually and eacin subject saw all the stimuli. Subjects were told that a sentence would appear in the center of the screen and that it would always be about "John" and "Henry". They were instructed to read the sentence silently and once having read the sentence to press a designated button to erase it from the screen. They were further instructed that following this leading sentence would be a sentence beginning with the pronoun "he". They were told that their task was to decide to whom the "he" referred. If "he" referred to "John" then they were instructed to press the button marked "John" and similarly if "he" referred to "Henry" they were instructed to press the button marked "Henry". They were encouraged to respond as quickly and accurately as possible. Short breaks between the presentation of blocks were permitted.

Results

This experiment was based on a mixed design with one between groups factor (Group) and one within groups factor (Preferred Referent). The factor Group had two levels -- younger adult (YA) and older adult (OA). The factor Preferred Referent had five levels -- NP1-Strongly Preferred (NP1-SP), NP1-Likely Preferred (NP1-LP), NP2Strongly Preferred (NP2-SP), NP2-Likely Preferred (NP2-LP), and Neutral (N).

Separate analyses with both subjects (F1) and items (F2) as random factors were performed on the mean reaction times for correct responses and on the proportion of errors. Min F' values were calculated and are only reported when significant. As in Pilot studies 
$2 \mathrm{a}$ and $2 \mathrm{~b}$, extreme reaction time values within each condition (those less than or greater than the condition mean $\pm 3 \times$ standard deviation) were replaced by that value. The number of responses replaced was approximately the same for all conditions ranging from $0.6-2.5$ $\%$ and $0.6-1.9 \%$ for the YA and OA groups, respectively.

Figure 6.1 shows subjects' mean reaction times to all sentence types for both groups. Analysis of the data by both subjects and items revealed significant main effects of Group $\left[\mathrm{E} 1(1,38)=16.93, \mathrm{R}<0.001 ; \mathrm{E} 2(1,35)=181.33, \mathrm{p}<0.001 ; \min \mathrm{F}^{\prime}(1,45)=15.48\right.$, D<0.01], with the OA group responding more slowly than the YA group overall, and Preferred Referent $\left[\mathrm{El}(4,152)=19.76, \mathrm{p}<0.001 ; \underline{\mathrm{F}} 2(4,35)=3.99,12<0.01 ; \min \mathrm{F}^{\prime}(4,50)\right.$ $=3.32, \mathfrak{2}<0.05]$. Pairwise comparison of the means for the latter condition using Newman-Keuls' procedure revealed that responses to sentences with both NP1 and NP2 as strongly preferred referents were faster than to neutral sentences, by both subjects and items $(p=0.05)$. No other differences were significant by both subjects and items. The interaction of Group by Preferred Referent was not significant by either subjects or items 8 .

Figure 6.2 shows the accuracy data for both groups of subjects. This analysis was only performed on responses to both the strongly and weakly preferred NP1 and NP2 sentences, thereby reducing the analysis to a $2 \times 4$ ANOVA. Obviously responses to the neutral sentences were not included as there was no correct response to these sentences. The analysis revealed a significant main effect, by both subjects and items, of Group $\left[\mathrm{E} 1(1,38)=9.37, \mathrm{p}<.01 ; \mathrm{E} 2(1,28)=11.93, \mathrm{p}<0.01 ; \min \mathrm{F}^{\prime}(1,66)=5.25, \mathrm{p}<0.05\right]$, with the OA group making more errors than the YA group. A main effect of Preferred Referent was also found, but by subjects only $[\mathrm{El}(3,114)=7.1, \mathrm{D}<0.001]$. Pairwise comparisons of the means using Newman-Keuls procedure revealed that fewer errors were made on sentences in which NP2 was the strongly preferred referent compared to all other sentence types. The interaction Group x Preferred Referent was not significant by either subjects or items 9 . 
Figure 6.1: Mean RT according to Preferred Referent

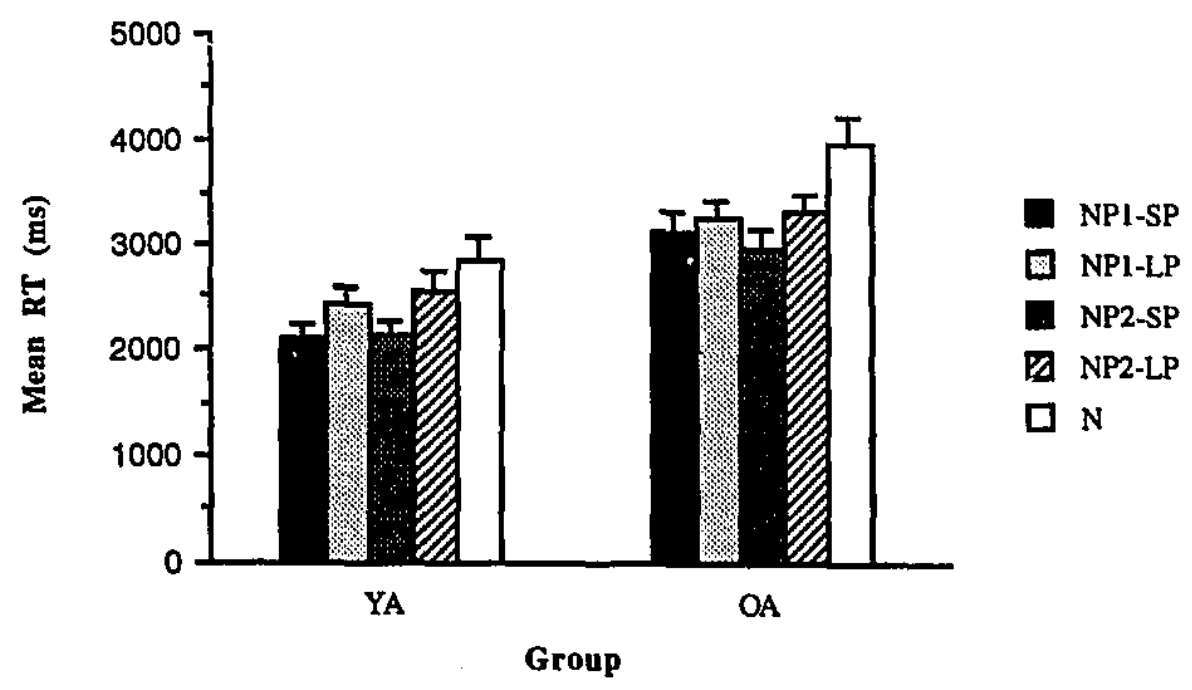


Figure 6.2: Mean \% errors according to Preferred Referent

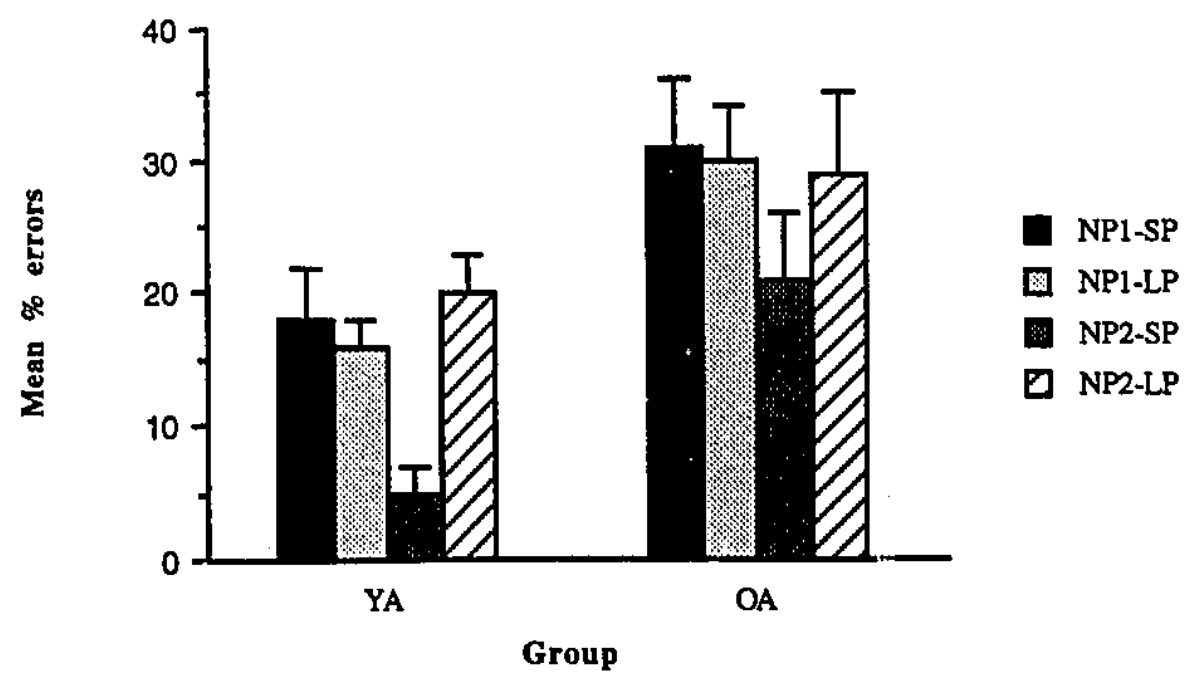


While the analysis of variance on the accuracy data (reported above) was useful in evaluating the proportion of errors as a function of strength of bias (i.e. strongly versus likely) of preferred referent (i.e. NP1 versus NP2), it did not indicate whether within each level of Preferred Referent, the preferred referent was, in fact, chosen significantly more often than chance. Therefore, following the procedure used by Hirst and Brill (1980), average response frequencies to preferred and non-preferred referents according to preferred referent status were calculated and are presented in Table 6.2. This value represents, proportionally, the number of times a referent was chosen out of a possible 8 opportunities, based on data from all subjects. A chi-square analysis was performed on the raw scores to compare the frequency of choice for the preferred referent versus the nonpreferred referent according to associated preferred referent status for each group separately. Analysis of the data for the YA group revealed significant differences between frequency of choice for preferred and non-preferred referents for all sentence types [NP1SP: $\mathrm{X}^{2}(1)=65.03, \underline{\mathrm{p}}<0.001 ;$ and NP1-LP: $\underline{\mathrm{X}}^{2}(1)=75.63, \underline{\mathrm{D}}<0.001 ; \mathrm{NP} 2-\mathrm{SP}: \underline{X}^{2}(1)=$ $129.6, \mathrm{p}<0.001$; and NP2-LP: $\mathrm{X}^{2}(1)=57.6, \mathrm{p}<0.001$ ]. Similarly, the OA group cho: the preferred referent significantly more often than the non-preferred referent for all sentence types [NP1-SP: $X^{2}(1)=24.03, \mathrm{D}<0.001$; and NP1-LP: $\underline{X}^{2}(1)=25.6, \mathrm{D}<0.001$; NP2-SP: $X^{2}(1)=55.23, \underline{p}<0.001 ;$ and NP2-LP: $\left.\underline{X}^{2}(1)=27.23, \mathfrak{p}<0.001\right]$. With respect to the neutral sentences, for which there was no preferred referent, choice of referent was approximately equally distributed to the first and second positions for the OA group [1st = $4.45,2$ nd $=3.55]$. However, the YA group demonstrated a preference for the referent in the first position $\left[1 \mathrm{st}=4.95,2 \mathrm{nd}=3.05 ; \mathrm{X}^{2}(1)=9.03, \mathrm{p}<0.01\right]$. The latter finding is, in fact, consistent with that of Hirst and Brill (1980) and can be attributed to the fact that the neutral sentences had a small negative plausibility rating overall, indicating that the referent in the first position was slightly preferred. 
Table 6.2

Average response frequencies to preferred (P) and non-preferred (NP) referents

\begin{tabular}{lccccc}
\hline & \multicolumn{2}{c}{ YA } & \multicolumn{2}{c}{ OA } \\
Preferred Referent & P & NP & P & NP \\
NP1-SP & & & & \\
NP1-LP & 6.55 & 1.45 & 5.55 & 2.45 \\
NP2-SP & 6.75 & 1.25 & 5.60 & 2.40 \\
NP2-LP & 7.60 & 0.40 & 6.35 & 1.65 \\
& 6.40 & 1.60 & 5.65 & 2.35 \\
& & & & & \\
\hline
\end{tabular}




\section{Discussion}

The results of this experiment serve as a replication of the findings of Hirst and Brill (1980). Similar patterns of response to those found in Hirst and Brill (1980) were demonstrated by both the YA and OA groups. With respect to the reaction time data, reaction times were faster to sentences in which there was a clearly biased referent than to neutral sentences. Similarly, with respect to the frequency of response data, the preferred referent was chosen significantly more often than the non-preferred referent by both groups of subjects. This overall pattern of results, therefore, supports the proposal by Hirst and Brill (1980) that the use of contextual information in light of general world knowledge is influential in determining antecedent choices for ambiguous pronouns.

Concerning the more interesting question addressed by this experiment -- the ability of older adults to use contextual information to pragmatically constrain the choices of referents for ambiguous pronouns -- the results are somewhat mixed. In terms of the reaction time data, there was no significant interaction between Group and Preferred Referent. The lack of an interaction indicated that the OA group exhibited the same pattern of response as that exhibited by the YA group in responding more quickly to sentences with a clearly specified preferred referent than to those with no preferred referent, when the correct choice was made. Similarly, in the analysis of the proportion of errors made, no interaction between Group and Preferred Referent was found, thereby indicating that both groups exhibited the same pattern of response to sentences according to preferred referent status. Specifically, subjects chose the preferred referent more often for sentences for which the second NP was the strongly preferred referent as compared to any of the other sentence types (note that this was only true with subjects as a random factor). However, overall, the OA group was also found to make significantly more errors than the YA group, reflecting a reduced sensitivity to the biasing information contained in the leading sentence. Thus, although as indicated by the analysis of the frequency of response data, OA were successful in using the biasing information in the leading sentence to choose the preferred 
referent more often than the non-preferred referent, overall they were not as successful as the YA group in doing so.

Overall, these results are consistent with those of LeDoux et al. (1983) and Light and Capps (1986) in that they provide evidence for the view that older adults are able to use contextual information in the resolution of pronouns. However, unlike the results of LeDoux et al. (1983) and Light and Capps (1986), the results of the present investigation also suggest that older adults are not as sensitive as younger adults to this information. Older adults were consistently found to choose the preferred referent less frequently than younger adults (in line with the findings of Light and Anderson, 1985) suggesting a reduced ability to use contextual information on the part of older adults.

A number of possible explanations are available to account for the discrepancy of these results. Perhaps, difficulty by the older adults in the present investigation was related to the taxing of working memory, since the leading sentence did not remain in view. As noted earlier, this procedure was in contrast to that used by LeDoux et al., (1983) in which both sentences remained in view until the subject made a response. Such an explanation is consistent with the finding that the ability to make inferences declines with older adults as working memory becomes sufficiently taxed (Light et al., 1982; Light \& Albertson, 1988; Zelinski \& Miura, 1990). On the other hand, one might argue that this explanation is not adequate given that in Light and Capps (1986) the stimuli were presented auditorily, thereby taxing working memory as well. Furthermore, it has been demonstrated that the ability of older adults to make inferences is usually more affected under auditory rather than visual presentation (Cohen, 1981; Zacks \& Hasher, 1988). Thus, while appealing to the notion of a reduced working memory capacity to explain the discrepancy between these results and those of LeDoux et al. (1983) is satisfactory, it is not completely adequate to explain the difference in these results compared to those of Light and Capps (1986).

Another possible explanation to account for the discrepancy of results between this study and those of Light and Capps (1986) relates to the fact that Light and Capps (1986) 
only used stimuli with strongly preferred referents (in addition to the neutral sentences). Perhaps, having a clearly preferred referent for the majority of the stimuli $[n=16]$ (the exception being the neutral stimuli $[n=8])$ may have more fully focused the subjects on the information contained in the leading sentence. Of course, this possibility is purely speculative. However, further support for this possibility is given by an inspection of the data by the younger adults for both studies. The proportion of errors for the younger adults tested in this study was considerably higher (mean $=12 \%$ for combined NP1 and NP2 strongly preferred sentences) than for those tested in Light and Capps (1986) (mean = $2 \%)$.

A third possibility to account for the discrepancy in results concerns the nature of the response. In this study, subjects were encouraged to respond as quickly as possible. In contrast, in LeDoux et al. (1983), accuracy above speed was encouraged and in Light and Capps (1986), speed of response was not even measured.

Finally, it should be noted that one canno completely discount the findings of Light and Anderson (1985) and the results of Pilot Study 2a wherein older adults were found to consistently perform worse than younger adults in identifying the referents of pronouns. The results of this study, in fact, corroborate these findings. Perhaps, in general, difficulty in resolving pronouns emerges as a function of aging. Certainly, in terms of production, there is abundant evidence to suggest that older adults have difficulty with pronominal reference (Cohen, 1979; North, Ulatowska, Macaluso-Haynes, \& Bell, 1986; Pratt, Boyes, Robins, \& Manchester, 1989; Ulatowska, Hayashi, Cannito, \& Fleming 1986).

To conclude, the results of this experiment confirm those of Hirst and Brill (1980) and provide evidence for the use of contextual information and pragmatic reasoning in the determination of referents for pronouns. Furthermore, these results indicate that older adults are also sensitive to this information, thereby supporting the results of LeDoux et al. (1983) and Light and Capps (1986). However, in contrast to these two studies, and in support of the findings by Light and Anderson (1985) and the results of Pilot Study 2a, the 
results of the present investigation also suggest that older adults are not as successful as younger adults in resolving pronouns. Thus, there continues to be a discrepancy concerning possible age differences in the resolution of pronouns. However, the key point to note with respect to the results of this study and the issue of aging, is that older adults were found to be sensitive to the pragmatic constraints provided by the information in the leading sentences in the resolution of ambiguous pronouns, exhibiting a pattern of response similar to younger adults. 


\section{Chapter 7: General Discussion for Pilot Studies}

A series of pilot studies was conducted in order to refine the stimuli to be used in the principal experiments with brain-damaged indi iduals. As well, the pilot studies were conducted in order to investigate possible age effects concerning the use of implicit causality of verbs and contextual information in resolving pronouns. It was important to establish that any effects of implicit causality and context found for younger adults would also be exhibited by older adults because the group of non brain-damaged controls to be used in the principal experiments would consist primarily of older adults.

The results of Pilot studies $1,2 \mathrm{a}$, and $2 \mathrm{~b}$ were successful in confirming the notion that older adults are as sensitive as younger adults to the implicit causality of verbs in pronoun resolution. In Pilot studies $2 \mathrm{a}$ and $2 \mathrm{~b}$ reaction times to sentences which set up an interpretation that was inconsistent with the verb's natural bias were slower than to sentences that were consistent with the verb's natural bias for both groups.

Additionally, the results of Pilot studies $2 \mathrm{a}$ and $2 \mathrm{~b}$ demonstrated that contextual information was influential in pronoun resolution, in terms of processing time, by setting up a discourse model by which to interpret pronouns. Importantly, this effect of context was also found for both younger and older adults. Thus, it was demonstrated that older adults were as efficient as younger adults in using preceding linguistic information to help determine the correct referents of ambiguous pronouns. However, the influence of context was not found to be sufficient to override the effects of the implicit causality of the verbs for either group.

The results of Pilot study 3 were important in establishing that both younger and older adults can use contextual information to pragmatically constrain preferred referents of pronouns. In contrast to the stimuli used in Pilot studies $2 \mathrm{a}$ and $2 \mathrm{~b}$, correct resolution of the ambiguous pronoun depended upon the use of the information contained in the leading sentence. As well, the information supportive of a particular referent was not specifically stated in the text. Rather, correct resolution of the pronoun was more dependent upon the 
use of general world knowledge. The results indicated that, like younger adults, older adults were sensitive to this information in their choice of preferred referents. However, there was also some indication that older adults were not as effective as younger adults in using this information as reflected in their higher error rates.

To summarize, the results of the pilot studies were crucial in establishing that older adults are sensitive to the effects of implicit causality and contextual information in the resolution of ambiguous pronouns. As a result of these investigations, one can be fairly confident that if the principal experiments demonstrate a lack of effect of either implicit causality, context or both by brain-damaged individuals, at the very least, the non braindamaged group will exhibit these effects. Thus, if any deficits are found to be characteristic of either brain-damaged group one will not simply be able to attribute them to the effects of aging. 


\section{Chapter 8: Experiment 1}

The purpose of Experiment 1 was to determine whether the property of verbs termed implicit causality, which has been found to be influential in the resolution of pronouns by younger adults (Caramazza et al., 1977; Garvey \& Caramazza, 1974; Garnham et al., 1992; Garvey et al., 1976; Grober et al., 1978; Vonk, 1985a; 1985b), older adults (Light \& Capps, 1983, cited in Light \& Albertson, 1988), and LBD individuals (Grober \& Kellar, 1981), also influences the ability of RBD individuals to resolve pronouns. Additionally, the investigation was extended to a group of LBD individuals and a group of non-brain damaged controls (NBD) in an attempt to corroborate these earlier findings. Importantly, this experiment was also specifically designed to explore the ability of RBD individuals to use contextual information at the level of the single sentence, in terms of the integration of information between clauses, in order to react and respond to the influence of implicit causality on the successful resolution of ambiguous pronouns. Details are elaborated below. It should be noted that this experiment is based on the norms determined by Pilot Study 1. However, unlike Experiments 2 and 3 which are direct replications of Pilot Studies 2a and 3, this experiment is different from Pilot Study 1 and is, in fact, based on the study by Caramazza et al. (1977).

As in Caramazza et al. (1977), subjects were required to identify the referent of pronouns in sentences such as "Mark confessed to Paul because he wanted forgiveness/offered forgiveness". In some sentences the interpretation produced by the reason provided in the subordinate clause was consistent with the verb's natural bias; in other cases, it was inconsistent with it. It was hypothesized that an effect for the implicit causality of verbs would be found for all three groups tested. However, the evidence for this effect was not necessarily expected to be the same for the three groups. The NBD group was expected to show a "normal" effect of implicit causality in terms of faster reaction times and possibly fewer errors to sentences that were consistent with the verb's 
natural bias than to those that were irconsistent with it. As just noted, both brain damaged groups were also expected to show an effect of implicit causality. The expectation for the LBD group was based primarily on the results of Grober and Kellar (1981) which demonstrated that LBD individuals were sensitive to this property of verbs. The expectation for the RBD group was based on the assumption that the implicit causality of a verb relates to the semantics of the verb and the fact that, apart from subtle difficulties, RBD individuals appear able to engage adequately in more basic linguistic processing.

The pattern of performance of the brain-damaged groups, however, was not necessarily expected to be the same as that predicted for the NBD group for the following reason. Reaction to the inconsistency of a reason to a verb's natural bias in terms of a slower reaction time, but a subsequent ability to successfully resolve the pronoun, requires the interpretation of the subordinate clause to be made in relation to the event depicted in the first clause. Based on the results of Grober and Kellar (1981), LBD individuals appear to have difficulty in integrating the information between the clauses in order to find the correct referent of a pronoun -- reflected in close to chance responding to inconsistent sentences. If these results are replicable, and LBD individuals really do evidence difficulty at this level, then a difference in reaction times to consistent and inconsistent sentences is not expected. Thus, the effect of implicit causality would only be evidenced in significantly more errors on inconsistent than consistent sentences. Similarly, given the possibility that RBD individuals have difficulty integrating information between clauses, it was conceivable that $\mathrm{RBD}$ patients would also not react to the inconsistent sentences, resulting in no difference, in terms of reaction times, to consistent and inconsistent sentences. However, as with the LBD group, an effect of implicit causality may still be manifested in more errors to inconsistent than consistent sentences.

Therefore, the finding of more errors on inconsistent than consistent sentences, but no difference between the sentences in terms of reaction times would suggest a sensitivity to the implicit causality of verbs, but difficulty in integrating information between clauses. 
On the other hand, the demonstration of the nomal pattern of implicit causality -- slower reaction times to and possibly more errors on inconsistent than consistent sentences -would be evidence for the use of contextual information, at least at the level of the single sentence.

To summarize, the purpose of Experiment 1 was twofold: 1) to determine whether or not the property of implicit causality influences pronoun resolution by RBD individuals; and 2) to investigate the ability of RBD individuals to use contextual information at the level of the single sentence in terms of the integration of information between clauses. Method

Subjects. Two groups of brain damaged subjects (right brain-damaged and left brain-damaged) were tested, as well as a group of non brain-damaged normal controls (see Table 8.1). The brain-damaged patients were recruited from a number of institutions in the Montreal, Ottawa and Boston areas. Initial exclusion criteria included multiple infarcts, known history of drug or alcohol abuse, history of psychiatric illness and/or neurological illness. The determination of lesion sites was based on neurological reports and Computerized Cranial Tomography (CT) scans when available. All patients had suffered a single cerebrovascular accident at the time of testing, mostly of an ischemic nature, although 5 right brain-damaged and 2 left brain-damaged patients were hemorrhagic. Lesions were primarily cortical with the exception of 1 right brain-damaged and 3 left brain-damaged patients who presented with primarily subcortical insults. All subjects were self-reported to be right-handed and native speakers of English.

The right brain-damaged (RBD) group consisted of 19 individuals ( 9 female and 10 male), ranging in age from $36-82$ years (mean age $=61.6$ years). Level of education ranged from 6 - 20 years (mean years of education $=12.9$ ). Eighteen of the patients were tested at least six months post onset. Based on available reports, none of the RBD patients presented with any obvious aphasic deficits ${ }^{10}$. 
Table 8.1

Summary of subject background information

\section{BBD}

\begin{tabular}{|c|c|c|c|c|c|}
\hline Patient & \begin{tabular}{|c|} 
Age \\
(years)
\end{tabular} & $\begin{array}{l}\text { Education }^{2} \\
\text { (years) }\end{array}$ & Sex & $\begin{array}{l}\text { Site of Lesion } \\
\text { (acc. to CT Scan) }\end{array}$ & Comments \\
\hline F.A. & 63 & 14 & F & $N^{b}$ & \\
\hline D.T. & 77 & NA & $\mathbf{M}$ & Normal in emergency & \\
\hline B.A. & 51 & 12 & $F$ & Posterior & hydrocephalus; \\
\hline W.K. & 78 & 17 & $\mathbf{M}$ & Parieto-occipital & \\
\hline J.S. & 71 & 10 & $\mathbf{M}$ & Parietal & \\
\hline A.M. & 50 & 9 & F & Temporo-parietal & hemorrhagic \\
\hline G.M. & 69 & 12 & $\mathbf{M}$ & Fronto-occipital & \\
\hline C.S. & 74 & 13 & $\mathbf{M}$ & Frontal & \\
\hline R.S. & 65 & 14 & $\mathbf{M}$ & Parieto-occipital & \\
\hline S.Z. & 56 & 13 & F & $\mathrm{MCA}^{\mathrm{C}}$ & hemorrhagic \\
\hline A.B. & 74 & 14 & F & Parietal & \\
\hline V.C. & 82 & 12 & F & NA & \\
\hline D.H. & 41 & 14 & $\mathbf{M}$ & MCA & hemorrhagic \\
\hline G.G. & 46 & 6 & $\mathbf{M}$ & $\mathrm{ACA}^{\mathrm{d}}$ & hemorrhagic \\
\hline M.C. & 62 & 9 & $F_{\text {: }}$ & Parietal & \\
\hline M.K. & 70 & 12 & F & Posterior & \\
\hline T.C. & 50 & 20 & $\mathbf{M}$ & NA & \\
\hline J.R. & 56 & 16 & $F$ & Fronto-temporal & \\
\hline D.G. & 36 & 16 & $\mathbf{M}$ & Basal ganglia & \\
\hline
\end{tabular}

"Best estimated conversion into years, based on information from subject (e.g. 2 years college, high school);

bNot available; ${ }^{C}$ Middle cerebral artery; ${ }^{d}$ Anterior cercbral artery 
Table 8.1 (cont'd)

\section{LBD}

\begin{tabular}{|l|l|l|l|l|l|}
\hline Patient & $\begin{array}{l}\text { Age } \\
\text { (years) }\end{array}$ & $\begin{array}{l}\text { Education } \\
\text { (years) }\end{array}$ & Sex & $\begin{array}{l}\text { Site of Lesion } \\
\text { (acc. to CT Scan) }\end{array}$ & Comments \\
\hline E.S. & 66 & 12 & F & MCA $^{c}$ & hemorrhagic \\
G.W. & 56 & 17 & M & Internal capsule & \\
J.W. & 82 & 8 & M & Frontal & \\
R.S. & 79 & 16 & M & Thalamus & hemornhagic \\
J.S. & 65 & 12 & M & Parietal & \\
B.C. & 42 & 14 & F & MCA & \\
I.B. & 72 & 11 & F & Basal nuclei & \\
D.I. & 44 & 14 & M & Parietal & \\
H.F. & 82 & 11 & M & Temporo-parietal & \\
I.G. & 78 & 11 & F & Parietal & \\
R.M. & 50 & 12 & M & Parietal & \\
S.C. & 81 & 10 & M & NA b & \\
\hline
\end{tabular}

Mean $66.4 \quad 12.3$

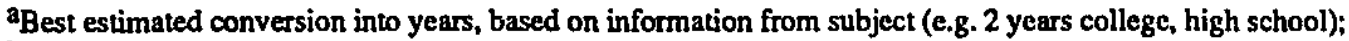
b Not available; "Middle cerebral artery 
Table 8.1 (cont'd)

NBD

\begin{tabular}{|l|l|l|l|}
\hline Subject & $\begin{array}{l}\text { Age } \\
\text { (years) }\end{array}$ & $\begin{array}{l}\text { Education } \\
\text { (years) }\end{array}$ & Sex \\
\hline G.C. & 65 & 13 & F \\
E.J. & 65 & 12 & M \\
F.U. & 55 & 14 & F \\
J.R. & 80 & 11 & M \\
Gw.B. & 70 & 13 & F \\
C.G. & 73 & 16 & M \\
G.B. & 70 & 12 & F \\
W.H. & 52 & 10 & F \\
E.C. & 78 & 16 & M \\
W.R. & 80 & 11 & M \\
D.E. & 78 & 11 & M \\
T.A. & 49 & 18 & M \\
D.F. & 77 & 12 & F \\
S.H. & 48 & 12 & F \\
J.R. & 40 & 14 & F \\
J.C. & 48 & 10 & M \\
M.Q. & 55 & 15 & F \\
P.K. & 52 & 11 & F \\
A.B. & 65 & 9 & F \\
\hline
\end{tabular}

Mean $\quad 63.2 \quad 12.6$

abest estimated conversion into years, based on information from subject (e.g. 2 years college, high school) 
The left-brain damaged (LBD) group consisted of 12 individuals (4 female and 8 male), ranging in age from $42-82$ years (mean age $=66.4$ years). Level of education ranged from $8-17$ years (mean years of education $=12.3$ ). All patients were tested at least six months post onset. Based on available speech and language reports, all of the patients presented with functional auditory comprehension skills and adequate reading abilities, at least at the level of the single word. As well, all but two (R.S. and S.C.) were noted to be aphasic. Patients comprising a range of aphasia classifications were included since it has been demonstrated that context effects for LBD individuals are not a function of aphasia type (Pierce \& Beekman, 1985; Pierce \& Wagner, 1985) ${ }^{11}$.

Screening battery. A number of screening tests were also administered to ensure that: a) none of the patients presented with any potentially confounding deficits related to neglect and single word reading impairments; and b) all of the patients presented with adequate auditory comprehension skills such that task instructions were likely to be understood. The screening battery consisted of the following tests:

1. Bells Test (BT) (Gauthier, Dehaut, \& Joanette, 1989):

This test was used to provide a measure of visual neglect. The subject was presented with a sheet of paper upon which were depicted the outlines of an assorted array of various small objects (e.g. guitars, saws, apples, trees, bells, etc.). The objects were organized on the paper in 7 columns, although the subject perceived them as being randomly ordered. Within each column 5 target objects (bells) and 40 distracters (e.g. guitars, saws, apples, etc.) were presented. Subjects were required to scan the array and circle all the bells. Performance was scored as follows. A failure to circle more than 3 bells was suggestive of an attentional deficit. A failure to circle six or more bells in the half of the page opposite a patient's lesion site (e.g. left for RBD patients) was highly suggestive of visual neglect. 
2. Written Word-Picture Matching (WWP) (subtest of the Psycholinguistic Assessment of Language (PAL) (Caplan, 1992)):

This test was administered to provide a measure of single word reading comprehension. On each trial the subject was presented with a choice of two black and white line drawings displayed on a sheet of paper ( $\left.81 / 2^{\prime \prime} \times 11 "\right)$. Printed below the two pictures was the target word. The subjects were instructed to point to the picture that best corresponded to the written word. A total of 32 word stimuli was used. The target words consisted of concrete nouns representing the categories of animals, fruits and vegetables, and tools.

3. Spoken Word-Picture Matching (SWP) (subtest of the PAL (Caplan, 1992)):

This test was administered to provide a measure of auditory word comprehension. The procedure was similar to that used for the Written Word-Picture Matching Test except that the target word was not printed below the two pictures but, rather, was presented orally by the examiner. The subject was required to point to the picture that best corresponded to the word spoken by the examiner. The stimuli were identical to those used in the written version. For this reason, these tests were usually administered on different days.

\section{Auditory Sentence Comprehension (ASC) (subtest of the PAL (Caplan, 1992)):}

This test was administered to provide a measure of auditory sentence comprehension. On each trial, the subject was presented with a choice of two black and white drawings displayed on a sheet of paper $\left(81 / 2^{\prime \prime} \times 11^{\prime \prime}\right)$. The subject was required to point to the picture that best corresponded to a sentence spoken by the examiner. A total of 20 stimuli were used. All were semantically reversible and consisted of four syntactic structures: active, passive, dative-passive, and subject-object relative.

In addition to the screening tests, another test (termed the Verb Test) was administered to all subjects (including control) to assess whether or not the meanings of the verbs used in the stimuli in Experiments 1 and 2 were known to the subjects. 


\section{Verb Test (VT):}

Three verbs were presented in a triangular formation. The target verb was presented at the top of the triangle, with two other verbs forming the base of the triangle (see below).

\section{PRAISE}

\section{GESTURE}

FLATTER

Of these two verbs, one was clearly related (in a synonymic fashion) to the target verb while the other was unrelated (see Appendix D for a list of the verbs and their response choices). Subjects were required to read the three words silently and to point to the verb that was most closely related to the target verb. Three practice items were administered prior to the test.

Performances by the brain-damaged subjects on the screening measures and on the Verb Test are presented in Table 8.2. As can be seen, members of both the LBD and RBD groups demonstrated adequate auditory word and sentence comprehension skills as well as good reading abilities at the level of the single word. In addition, the results of only one RBD patient suggested the possibility of neglect. However, upon consideration of her very low error rate on all the experimental tasks, it was felt that the possibility of neglect was unlikely. Concerning the results of the Verb Test, one RBD patient, 5 LBD patients, and one NBD control made errors on at least one verb. These errors were taken into account in the analysis of the data for both Experiments 1 and 2. As will be seen, they did not figure in the interpretation of the final results. 
Table 8.2

Summary of performances on screening tests:

BT (Bells Test); ASC (Auditory Sentence Comprehension); SWP (Spoken Word-Picture Matching); WWP (Written Word-Picture Matching); VT (Verb Test)

\section{RBD}

\begin{tabular}{|l|l|l|l|l|l|}
\hline Patient & BT 35 & ASC $/ 20$ & SWP $/ 32$ & WWP 32 & VT $/ 20$ \\
\hline F.A. & $31^{\mathrm{a}}$ & DNA & DNA & DNA & 20 \\
D.T. & 34 & 18 & 32 & 32 & 20 \\
B.A. & 34 & 19 & 32 & 32 & 20 \\
W.K. & 33 & 18 & 31 & 32 & 20 \\
J.S. & 33 & 15 & 32 & 32 & 19 \\
A.M. & $30^{\mathrm{a}}$ & 15 & 32 & 32 & 20 \\
G.M. & 34 & 17 & 32 & 32 & 20 \\
C.S. & 34 & 20 & 32 & 32 & 20 \\
R.S. & 32 & 20 & 32 & 32 & 20 \\
S.Z. & $30^{\mathrm{a}}$ & 15 & 31 & 32 & 20 \\
A.B. & 33 & 19 & 30 & 30 & 20 \\
V.C. & 35 & 19 & 31 & 30 & 20 \\
D.H. & $27^{\mathrm{a}}$ & 18 & 32 & 32 & 20 \\
G.G. & 34 & 19 & 32 & 32 & 20 \\
M.C. & $31^{\mathrm{a}}$ & 17 & 32 & 32 & 20 \\
M.K. & 33 & 20 & 32 & 32 & 20 \\
T.C. & 32 & 20 & 32 & 32 & 20 \\
J.R. & $27^{\mathrm{d}}$ & 20 & 32 & 32 & 20 \\
D.G. & 35 & 20 & 31 & 31 & 20 \\
Mean & & 18.3 & 31.7 & 31.7 & 19.9 \\
\hline
\end{tabular}

${ }^{a}$ Possible attentional deficit; ${ }^{b}$ Did not administer; ${ }^{c}$ Administered by phone;

dPossible neglect; however, given extremely low error rates on experimental tasks, neglect is unlikely. 
Table 8.2 (cont'd)

\section{LBD}

\begin{tabular}{|l|l|l|l|l|l|}
\hline Patient & BT /35 & ASC /20 & SWP /32 & WWP /32 & VT /20 \\
\hline E.S. & 35 & DNA $^{\text {b }}$ & DNA & DNA & 16 \\
G.W. & 35 & 17 & 32 & 32 & 20 \\
J.W. & 35 & 18 & 32 & 32 & 20 \\
R.S. & 33 & 18 & 32 & 32 & 20 \\
J.S. & 34 & 13 & 30 & 30 & 14 \\
B.C. & 35 & 17 & 32 & 32 & 20 \\
I.B. & 35 & 16 & 32 & 32 & 20 \\
D.I. & 35 & 12 & 32 & 32 & 20 \\
H.F. & 32 & 15 & 32 & 32 & 20 \\
I.G. & 35 & 14 & 32 & 30 & 17 \\
R.M. & $29 \mathrm{a}$ & 20 & 32 & 31 & 18 \\
S.C. & 35 & 16 & 32 & 29 & 19 \\
\hline
\end{tabular}

apossible attentional deficit; ${ }^{\mathrm{b}}$ Did not administer;

*This value is significantly different from that for the $R B D$ group $[(27)=2.93, \underline{p<0.01]}$ 
The non brain-damaged (NBD) control group consisted of 19 individuals (11 females and 8 males). These subjects were chosen from a large number of volunteers in the Montreal area. Control subjects were matched as closely as possible to members of the $\mathrm{RBD}$ group on the variables of sex, age and education. Fortunately, both brain-damaged groups also turned out to be well matched on these variables, therefore, the NBD group was also closely matched to the LBD group. The age of the NBD individuals ranged from $40-80$ years (mean age $=63.2$ years) and level of education from $9-18$ years (mean years of education $=12.6$ ). All control subjects were self-reported to be right-handed and native speakers of English and none had a known history of neurological or psychiatric illness. As well, all subjects had to pass a series of neuropsychological tests, which included the Boston Naming Test (Kaplan, Goodglass \& Weintraub, 1983), a modified version of the Mini-Mental State Examination (Folstein, Folstein, \& McHugh 1975) and Logical Memory subtests (Immediate and Delayed) of the Wechsler Memory Scale Revised (Wechsler, 1987), to rule out the possibility of other disorders such as dementia, before being considered suitable for testing ${ }^{12}$.

Materials and Apparatus. The stimuli used were similar, and at times identical (except for the names), to those used by Caramazza et al. (1977) and consisted of sentences of the form NPI Verb(ed) NP2 because Pronoun Reason (e.g. Mark lost to Paul because he was a poor player). Two factors were manipulated in the construction of the stimuli. The first factor, Ambiguity, related to whether or not the pronoun was ambiguous based on gender cues. It had two levels -- ambiguous (A) and unambiguous (U). In the ambiguous sentences, both NP1 and NP2 were of the same gender, rendering the pronoun ambiguous. In contrast, in the unambiguous sentences, NP1 and NP2 were of different genders allowing for the pronoun to be disambiguated based on this information alone. The second factor, Verb Consistency, related to the consistency of the interpretation produced by the information in the subordinate clause to the verb's natural bias. It also had two levels -- verb-consistent (VC) and verb inconsistent (VI). The resulting combination 
of sentences and an example of each is provided in Table 8.3 (see Appendix $E$ for a complete listing of the stimuli).

A total of eighteen verbs was used ( 9 NP1 and 9 NP2), each of which was found to have a percentage of dominant response equal to or greater than 70 in Pilot Study 1, resulting in a total of 72 sentences. It should be noted that for the unambiguous sentences, the gender of either NP1 or NP2 was changed such that the final clause remained unchanged across all four sentences. The mean lengths of the VC and VI sentences for each verb were matched in terms of number of words. The mean lengths of these sentences were 9.05 and 9.0 , respectively. It was important that the sentences were matched in terms of length since it was the reaction times to these sentences that was the crucial dependent variable.

The sentences were divided into 4 blocks with only one instance of each verb represented in each block. The sentences within each block were randomly ordered and the order of presentation of the blocks was counterbalanced across subjects. Ten practice sentences preceded the presentation of the experimental stimuli.

The sentences were presented one at a time in the center of a computer screen. Below each sentence were the names of the two people mentioned in the sentence, in the same order that they were presented. Subjects responded by pressing one of two designated buttons, situated adjacent to one another, on a response box that was interfaced with the computer. The buttons corresponded to either the first or second person mentioned in the sentence and represented the potential referents for the pronoun. Once a response was made, the next stimulus item appeared in the center of the screen. All responses were made with a finger of the right hand, for the controls, or of the least impaired hand, for the patients ${ }^{13}$. The computer recorded both reaction time (with a time base of 0.001 seconds) and accuracy. 


\section{Table 8.3}

Examples of Stimuli for Experiment 1

Note that the verb used in these stimuli, lost to, is an NP1 type verb.

A-VC Mark lost to Paul because he was a poor player.

A.VI Mark lost to Paul because he was a great player.

U-VC Mark lost to Beth because he was a poor player.

U-VI Beth lost to Mark because he was a great player. 


\section{Procedure.}

Subjects were tested individually and each subject saw all the stimuli. Prior to the administration of the experimental task two preliminary tests were administered. First, subjects were given a task to ensure that they could read simple sentences from the computer and to obtain a crude measure of subjects' general mental orientation. The test consisted of five simple questions presented visually in the center of a computer screen. The following questions were used: "What is your name?", "What day of the week is it?", "What month is it?", "How old are you?", "What season of the year is it?". Subjects were told that this test was to ensure that they could see the screen clearly. They were instructed to read the sentence silently and to answer it out loud. Once they answered the question, they were instructed to press a designated button on the response box to advance to the next question. Responses were scored as either correct or incorrect by the examiner. Once it was established that the subjects could read from the screen satisfactorily they were administered a second preliminary test.

The second preliminary test was administered primarily to ensure that the subjects were capable of associating a particular button on the response box with a position (i.e. first or second) on the computer screen ${ }^{14}$. This skill was necessary for responding accurately in the experimental task. In addition, this task gave the subjects added practice in the type of responding necessary for the experimental task. The test involved the presentation of an "X" and an "O" (or vice versa), side by side, in the center of the computer screen. Subjects were told that this test was simply to familiarize them with the response buttons. Subjects were required to respond according to where the "O" was on the screen. If it was on the left, they were instructed to press the left button of the response box; if it was on the right, to press the right button. Reaction time and accuracy were recorded by the computer. If it was obvious to the experimenter that the subject could not perform the task, then no further testing was conducted. Two subjects were excluded on this basis ${ }^{15}$. All subjects included in the experiment performed this task with at least $90 \%$ 
accuracy. In fact, only 1 LBD subject, 5 RBD subjects and 2 NBD subjects made any errors at all.

Next, the experimental task was administered. During the instructional phase of the task, the subjects were presented with demonstration stimuli on the computer ${ }^{16}$. These examples were referred to when the examiner was issuing the instructions. Subjects were told that a sentence would appear in the center of the screen. They were instructed that in each sentence two people would be mentioned and a pronoun, a "he" or a "she" would be used. It was pointed out to the subjects that the names of the two people mentioned were listed below the sentence in the same order in which they appeared in the sentence. Subjects were told that their task was to decided to whom the pronoun (the "he" or the "she"') referred and to indicate their choice by pressing the appropriate button on the response box, corresponding to the position of the person in the sentence (i.e. left button for the first person mentioned and right button for the second person mentioned). They were encouraged to respond as quickly and accurately as possible. Short breaks were permitted between the presentation of blocks.

Results

The experiment used a mixed design with one between groups factor and two within groups factors. The between groups factor was Group and it had three levels -right brain-damaged (RBD), left brain-damaged (LBD) and non brain-damaged (NBD). The two within groups factors were Ambiguity, with two levels - ambiguous (A) and unambiguous (U); and Verb Consistency, also with two levels - verb consistent (VC) and verb inconsistent (VI).

An analysis was performed which excluded the data for sentences that contained verbs to which subjects responded incorrectly on the verb test. Since the results of this analysis were consistent with the results of the analysis that used the complete data set, only the results based on the complete data set are reported. 
For this experiment, as well as for Experiments 2 and 3, separate analyses of variance with both subjects $(\mathrm{F} 1)$ and items $(\mathrm{F} 2)$ as random factors were performed on the logarithm of the mean reaction times and the square root of the proportion of errors. Min $F^{t}$ values were also calculated and are reported when significant. Extreme reaction time values within each condition (those less than or greater than the condition mean $\pm 3 x$ standard deviation, calculated per subject for correct responses only) were replaced by that value prior to the transformation of the data. The number of responses replaced in the present experiment was approximately the same across conditions and groups, ranging from $0-0.7 \%, 0-1.7 \%$, and $0.4-1.0 \%$ for the RBD, LBD, and NBD groups, respectively. Also, during testing for all three experiments, the experimenter was alert for instances in which the subject was not attending to the task (for example, talking, sneezing, telephone interruptions, etc.) or for instances for which the computer did not register a response (for example, if the button was not pressed with sufficient force). These instances were recorded as mechanical errors and were not included in the analyses. Additionally, reaction times greater than 30 seconds were automatically timed out by the computer. Responses to items timed out were also not included in the analyses. The number of mechanical errors and responses automatically timed out for the present experiment accounted for only $2.6 \%, 3.1 \%$, and $0.41 \%$ of the total number of possible responses for the RBD, LBD, and NBD groups, respectively 17 .

Based on the analysis of the accuracy data (reported below), RBD subjects J.S. and G.G. were found to have pressed the same button in response to all of the stimuli. As such, their data were excluded from the analyses reported for this experiment. To be consistent, the data from their matched controls (Gw.B. and J.C., respectively) were also excluded.

Figure 8.1 shows subjects' mean reaction times to verb consistent and verb inconsistent sentences under both conditions of ambiguity for all groups. Analysis of the data by both subjects and items revealed a significant main effect of Group $[\mathbb{E} 1(2,43)=$ 
$\left.14.74, \mathfrak{p}<0.001 ; \mathrm{E2}(2,136)=487.91, \mathfrak{p}<0.001 ; \min \mathrm{F}^{\prime}(2,46)=14.31, \mathfrak{p}<.01\right]$. Pairwise comparisons of the group means using Newman-Keuls' procedure revealed that responses by the NBD group (mean $=4517 \mathrm{~ms}$ ) were significantly faster than those by both the RBD $(7029 \mathrm{~ms})$ and LBD (mean $=9421 \mathrm{~ms})$ groups, by both subjects and items $(p=0.05)$. As well, reaction times by the RBD group were significantly faster than those by the LBD group, by both subjects and items $(p=0.05)$. Significant main effects of Ambiguity $\left[\mathrm{El}(1,43)=38.16, \mathfrak{p}<0.001 ; \mathrm{E} 2(1,68)=36.22, \mathfrak{p}<0.001 ; \min \mathrm{F}^{\prime}(1,107)=18.58\right.$, D $<0.01$, with reaction times to ambiguous sentences slower than to unambiguous sentences, and Verb Consistency $[\mathrm{E} 1(1,43)=40.70, \mathfrak{p}<0.001 ; \mathrm{F} 2(1,68)=9.47, \mathfrak{p}<0.01$; $\left.\min F^{\prime}(1,95)=7.68, \mathfrak{p}<0.01\right]$, with reaction times slower to VI than to VC sentences, were also found. No interactions were found to be significant by both subjects and items.

The reaction time data for each individual subject was also examined to determine whether the individuals of each group also demonstrated a pattern of response consistent with an effect of implicit causality. The difference in mean reaction times to VC and VI sentences was calculated and presented as a proportion of the mean reaction time to VC sentences [i.e. (VI-VC)/VC], per subject, to provide a measure of the mean percentage increase in reaction times to VI compared to VC sentences. This measure was calculated on the means for ambiguous and unambiguous sentences separately. The results of the analysis are presented in Figure 8.2. A positive value indicates that the mean reaction time to VI sentences was slower (i.e. increased) than to VC sentences; and a negative value indicates the reverse. Upon inspection of the graphs, the important point to note is that, while some of the differences are not large, the majority of subjects from all the groups responded more slowly to VI than to VC sentences. In fact, only two LBD subjects (J.S. and B.C.), two RBD subjects (A.M. and R.S.) and one NBD subject (A.B.) responded more slowly to VC than VI sentences for both ambiguous and unambiguous types. 
Figure 8.1: Mean RT to VC and VI sentences according to Ambiguity RBD

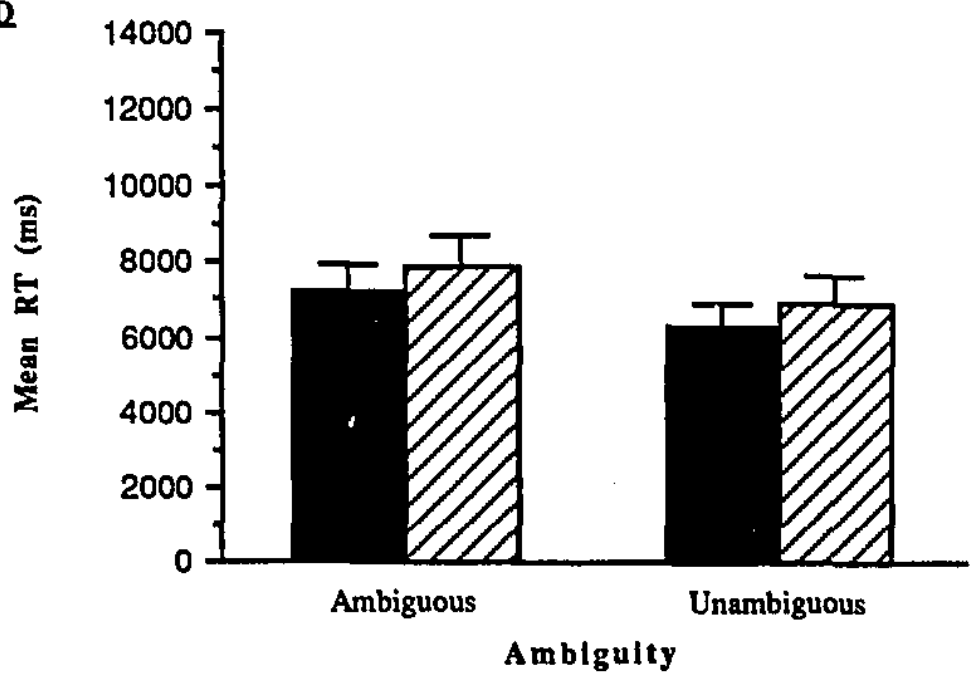

LBD

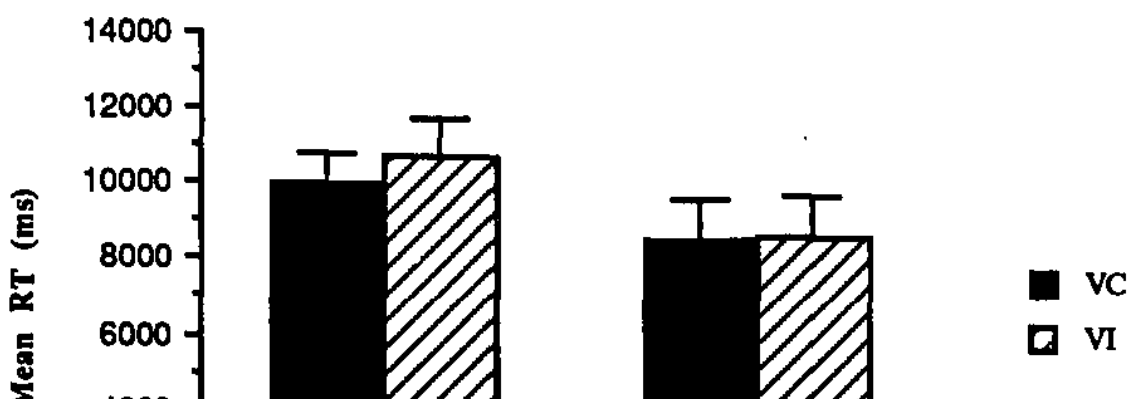

NBD

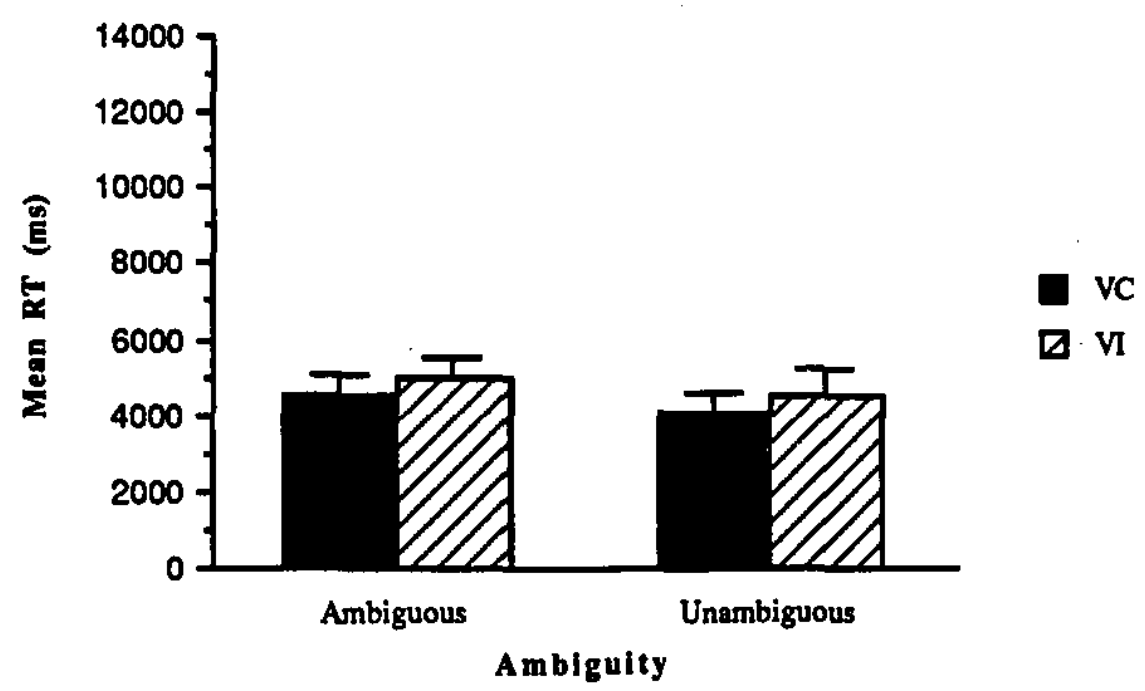


Figure 8.2: Mean \% increase in RT to VI compared to VC sentences
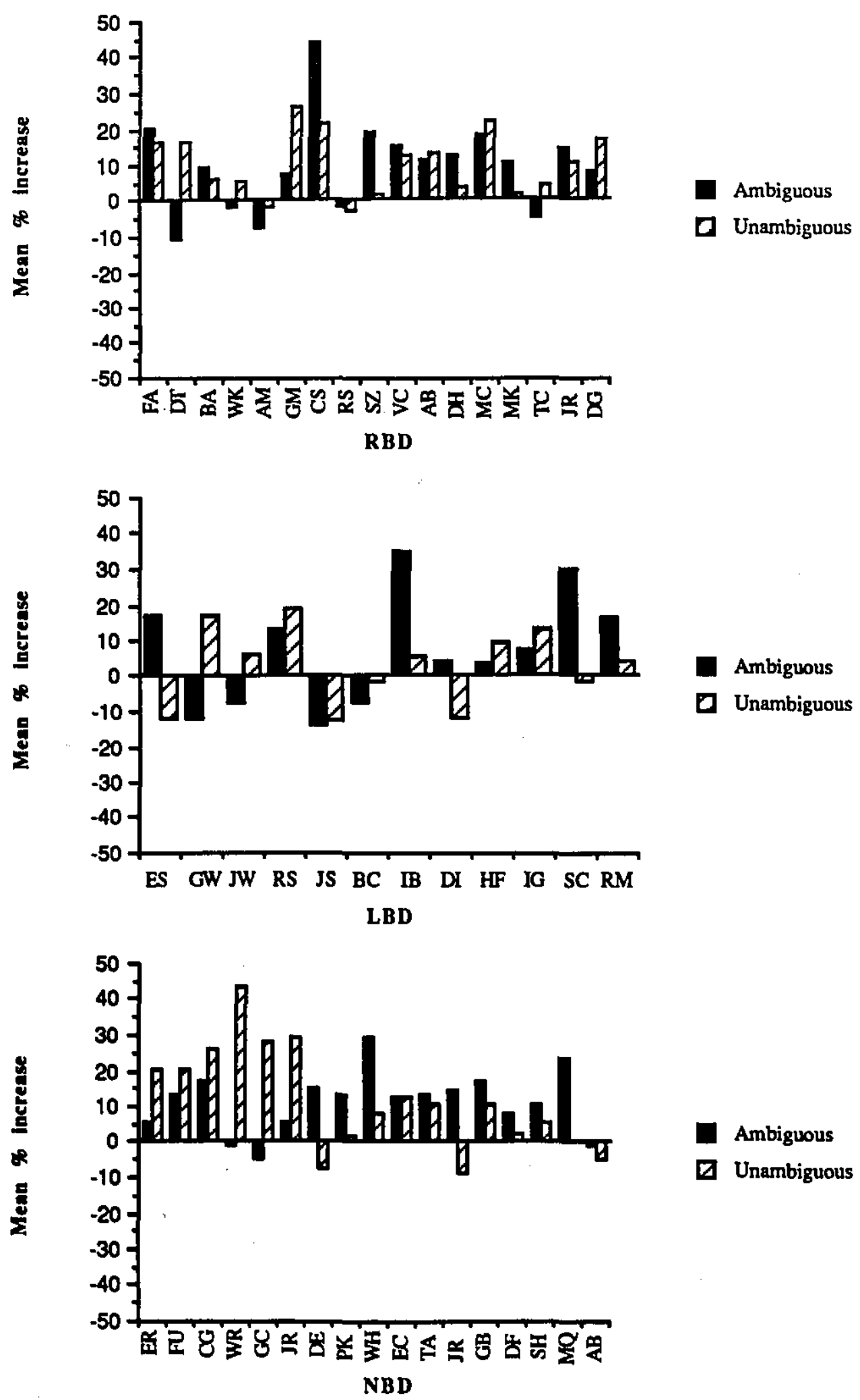
Figure 8.3: Mean $\%$ errors to VC and VI sentences according to Ambiguity RBD

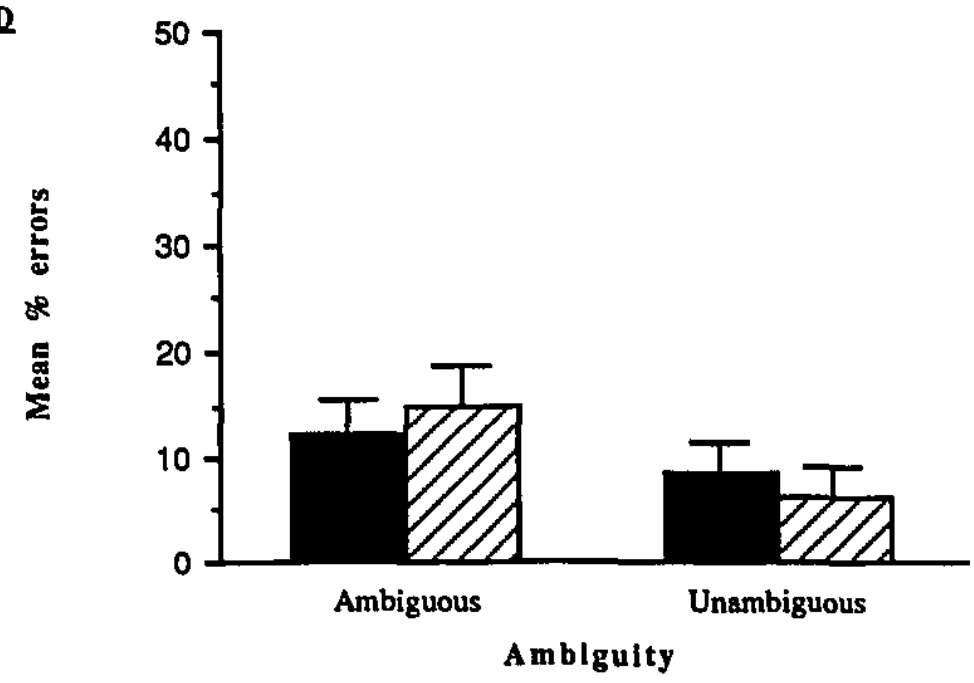

LBD

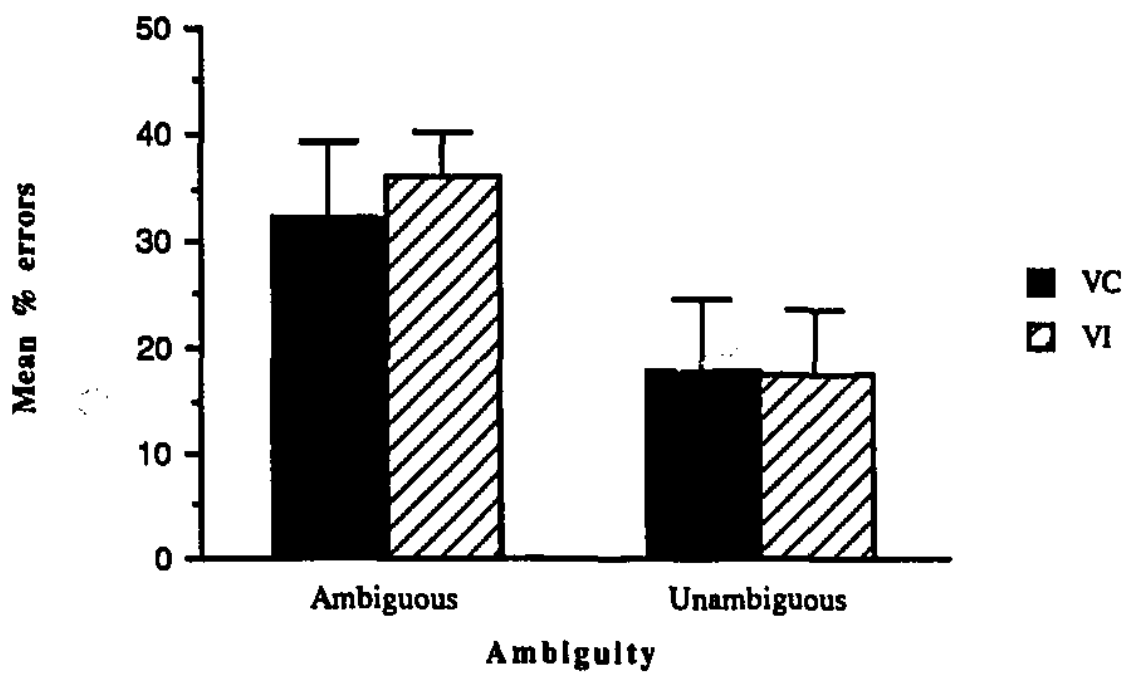

NBD

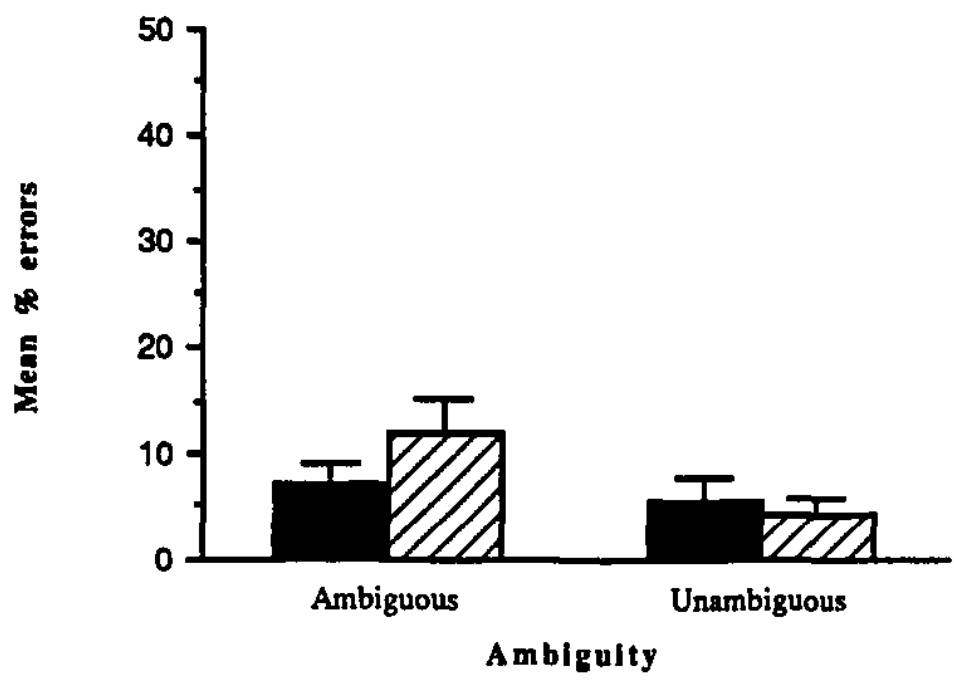


The accuracy data are represented in Figure 8.3. A significant main effect of Group $\left[\mathrm{E} 1(2,43)=7.17, \mathrm{D}<0.01 ; \mathrm{E} 2(2,136)=73.90, \mathrm{R}<0.001 ; \min \mathrm{F}^{\prime}(2,52)=6.54, \mathbb{R}<0.01\right]$ was found. Pairwise comparisons of the means using Newman-Keuls' procedure revealed that the LBD group (mean \% errors $=26$ ) made significantly more errors than both NBD (mean \% errors $=7$ ) and RBD groups (mean \% errors $=10$ ), who were not significantly different from each other, by both subjects and items $(p=0.01)$. A significant main effect of Ambiguity $\left[E 1(1,43)=25.44, \mathrm{R}<0.001 ; \mathrm{E} 2(1,68)=40.6, \mathrm{R}<0.001 ; \underline{\min } \mathrm{F}^{\prime}(1,91)=\right.$ $15.6, \mathfrak{p}<0.01]$ was also found, with more errors being made on ambiguous than unambiguous sentences. The interaction Ambiguity $\mathbf{x}$ Verb Consistency was also found to be significant $[E 1(1,43)=4.78, \mathbb{D}<0.05 ; E 2(1,68)=5.42, \mathbb{R}<0.05]$. However, the analysis of the simple main effects proved uninteresting. The factor Ambiguity was found to be significant at both levels of Verb Consistency, while Verb Consistency was not significant by both subjects and items at either level of Ambiguity.

\section{Discussion}

The results of this experiment provide further support for the robustness of the effect of implicit causality in resolving pronouns. As expected, the assignment of referents by NBD individuals was found to be significantly slower when the interpretation provided by the information in the subordinate clause was inconsistent with the bias imputed by the verb. As in Caramazza et al. (1977), while responses were faster to unambiguous than ambiguous sentences, the factor of ambiguity did not interact with verb consistency, thereby corroborating the finding that the effect of iraplicit causality holds even when disambiguation of the pronoun is possible based solely on linguistic cues (in this case gender cues).

More interesting to the purposes of this study, however, was the finding that the property of implicit causality was also influential in determining coreference for both the LBD and RBD groups. While overall the NBD group responded faster than both braindamaged groups, both the LBD and RBD groups exhibited the same pattem of response as 
the NBD group; namely, faster responses to unambiguous than ambiguous sentences and slower responses to verb inconsistent than verb consistent sentences. Moreover, a pattern of response reflecting an effect of implicit causality was also demonstrated individually, by most subjects of each group, for at least one level of ambiguity.

With respect to the LBD group, these results partially corroborate the findings of Grober and Kellar (1981) in that both studies found evidence to suggest that LBD individuals are sensitive to the property of implicit causality when assigning coreference. However, the results of the accuracy data of the present study are somewhat inconsistent with those of Grober and Kellar (1981). Recall that in the Grober and Kellar (1981) study (which only looked at accuracy data, not reaction time data), there was some suggestion that LBD individuals have difficulty integrating information between clauses which was reflected in close to chance responding to ambiguous verb inconsistent sentences. Based on this finding, it was hypothesized for the present study that LBD individuals might not evidence an effect of implicit causality in terms of the reaction time data since a slower reaction to verb inconsistent sentences requires a recognition of the inconsistency of the information in the subordinate clause with the information in the first clause. However, it was reasoned that a sensitivity to the implicit causality of verbs may still be evidenced by LBD individuals in terms of significantly more errors on ambiguous verb inconsistent than verb consistent sentences. This hypothesis, in fact, failed to be supported on two counts. First, as just noted, the LBD group was found to respond more slowly to verb inconsistent than verb consistent sentences for both ambiguous and unambiguous sentences. Second, there was no significant effect of verb consistency in terms of the accuracy data for any of the groups. Moreover, upon closer inspection of the mean proportion of errors made by LBD individuals, it was found that responses to ambiguous verb inconsistent sentences were not simply at chance as they were in Grober and Kellar (1981). While overall the LBD group made significantly more errors than both the NBD and RBD groups, LBD individuals were able to choose the correct referent of the pronoun of verb inconsistent 
sentences $64 \%$ of the time (as compared to $68 \%$ accuracy to verb consistent sentences). Additionally, similar to the pattern of response exhibited by the NBD and RBD groups, they also made significantly fewer errors to unambiguous than ambiguous sentences.

Thus, although the results of both the present study and those of Grober and Kellar (1981) support the notion that LBD individuals are sensitive to the property of implicit causality in determining coreference, there is a discrepancy between the two studies with respect to the abilities of LBD individuals to integrate information between clauses. The present results suggest that LBD individuals are capable of successfully integrating information between clauses, albeit not to the same extent as NBD or RBD individuals. Certainly it is not surprising to find, given their linguistic deficits, that LBD individuals have considerably more difficulty in resoiving pronouns than either NBD or RBD individuals. However, the important point to note is that when the referent of a pronoun is correctly assigned, LBD subjects are sensitive to the implicit causality of the verb and the inconsistency of this bias with the interpretation established in verb inconsistent sentences, thus demonstrating an ability to integrate information between clauses. It is not clear why the results of the present study differ from those of Grober and Kellar (1981) concerning this ability. Perhaps the discrepancy relates to the nature of the task. In Grober and Kellar (1981), subjects were asked a question querying the referent of the pronoun and were always provided with the same four referent choices in picture form. It is possible that by always using the same potential referents the task was made more confusing.

Another possibility to account for the discrepancy between the two studies concerns the severity of the comprehension impairment of the patients tested. The LBD patients in the present study were quite high functioning, as necessitated by the demands of the task, and as reflected in their relatively good performance on the auditory sentence comprehension test of the screening battery. In contrast, of the 13 aphasic patients tested by Grober and Kellar (1981), 5 were characterized as presenting with a mild-moderate comprehension deficit, while the remaining 8 were judged to present with a severe 
comprehension deficit. Thus, the apparent inability to integrate information between clauses by the LBD patients tested in Grober and Kellar (1981) may have been a function of the existence of more severe auditory comprehension deficits in the patients they tested as compared to those tested in the present study. However, this possibility is not supported by the results of the accuracy of response data to ambiguous verb-consistent sentences. The LBD patients in Grober and Kellar (1981) made fewer errors than those of the present study ( $27 \%$ versus $32 \%$, respectively) on these types of sentences. It is also of interest to note that they made fewer errors, compared to the LBD patients of the present study, on the unambiguous verb-consistent sentences as well (13\% versus $18 \%$, respectively), but more on the unambiguous verb-inconsistent sentences (23\% versus $17 \%$, respectively). Given, therefore, that the LBD patients of Grober and Kellar (1981) did not perform worse than those of the present study on all sentence types, an explanation based on the possibility of a more severe auditory comprehension impairment is not sufficient to explain why the LBD patients of Grober and Kellar (1981) exhibited an inability to successfully integrate information between clauses. Once again, however, the important point to emphasize is that the results of both the present study and those of Grober and Kellar (1981) were consistent in demonstrating a sensitivity on the part of LBD patients to the implicit causality of verbs in the resolution of pronouns.

The pattern of response by the RBD group is revealing with respect to a number of points. First, it is of interest to note that although RBD individuals responded more slowly than the NBD group, they did not make significantly more errors than NBD subjects. These findings support the view that the syntactic abilities of RBD individuals are essentially intact. Second, the results convincingly support the notion that a sensitivity to the property of implicit causality is preserved in RBD individuals. As just noted, similar to the response patterns for both the NBD and LBD groups, reaction times to verb inconsistent sentences were slower than to verb consistent sentences for both ambiguous and unambiguous sentences. Moreover, this pattern of response appears quite stable as it 
was found to be true for 12 of the 17 patients tested. Additionally, another 3 patients demonstrated this pattem of response for unambiguous sentences only. Importantly, the finding that the evidence for the preservation of implicit causality by the RBD group emerged from the reaction time data is telling with respect to the ability of RBD individuals to use contextual information. Recall that it has been argued that in order to react to the inconsistency of an interpretation with a verb's natural bias, in terms of a slower reaction time, but a subsequent ability to resolve the pronoun, the inconsistent information provided in the subordinate clause must be integrated with the information in the first clause and the relationship between the two determined. The fact that the RBD group demonstrated a clear ability to do so suggests that, at the very least, RBD individuals are capable of using contextual information at the level of the single sentence in terms of the integration of information between clauses.

Interestingly, these results also speak to an issue not specifically addressed by this study. Evidence from a number of studies (e.g. Brownell et al., 1986; Schneiderman \& Saddy, 1988; Ulatowska \& Baker, 1976) has suggested that RBD individuals may be impaired in their ability to revise initial interpretations. For example, in Brownell et al. (1986), it was found that RBD patients exhibited difficulty in revising initial inferences that were based on misleading information once the informative information was encountered. The authors suggested that such a finding may be due to a difficulty on the part of RBD patients to reject initial interpretations. The present results, however, suggest that RBD individuals are capable of rejecting and revising initial interpretations, at least with respect to the semantic factor of implicit causality. In the present study, if RBD patients liad been unable to reject the initial interpretation imputed by the bias of the verb, they should have made considerably more errors on ambiguous verb inconsistent than verb consistent sentences. In fact, this was not the case and their error rate for these sentences was comparable to that of NBD controls ( $15 \%$ vs. $12 \%$, respectively). 
To summarize, the results of this study corroborate the finding that the property of verbs termed implicit causality is influential in pronoun resolution for both NBD controls (Caramazza et al., 1977) and LBD individuals (Grober \& Kellar, 1981). Additionally, the results extend this finding and demonstrate that this property of verbs also influences the resolution of pronouns by RBD individuals. Furthermore, important to the question of the ability of brain damaged individuals to use contextual information, these results suggest that both LBD and RBD individuals are capable of using contextual information, in terms of the integration of information between clauses, in the successful resolution of pronouns. 


\section{Chapter 9: Experiment 2}

Experiment 1 was successful in demonstrating that RBD patients can use contextual information at the level of the single sentence -- in terms of the integration of information between clauses. The next logical step was to determine whether or not RBD individuals could use contextual information at the level of a minimal discourse (i.e. two sentences) as well. Based on the results of Pilot Studies $2 a$ and $2 b$, it is clear that contextual information, at the level of a minimal discourse, does influence pronoun resolution. Having established this phenomenon empirically in normal non brain-damaged individuals (including older adults), therefore, it was of interest to determine whether or not brain-damaged, and in particular right brain-damaged individuals, would exhibit the same pattern. The primary purpose of Experiment 2, therefore, was to examine the use of contextual information by RBD patients in resolving pronouns, and, importantly, in contrast to Experiment 1, to address the abilities of RBD patients to use contextual information across sentence boundaries. As in Experiment 1, the study was extended to a group of LBD patients and a group of non brain-damaged controls.

It was hypothesized that both the NBD controls and the LBD group would show an effect of context reflected in faster reaction times to determine the referent of an ambiguous pronoun for sentences preceded by a supportive context as compared to sentences not preceded by any context. The hypothesis for the NBD group was based on the results of Pilot studies 2a and 2b. The hypothesis for the LBD groups was based on the mounting evidence (e.g.Cannito et al,, 1986; Friederici, 1983; Germani \& Pierce, 1992; Hough et al., 1989; Pierce \& Beekman, 1985; Pierce \& DeStefano, 1987; Pierce \& Wagner, 1985; Waller and Darley, 1978) that suggests that LBD patients use preceding linguistic information to a considerable degree to aid in language processing.

In contrast, based on the prevailing view that a difficulty in using contextual information may be the underlying deficit for the discourse level impairments exhibited by 
RBD patients (Cook, 1989a; Gardner et al., 1983; Wapner et al., 1981), RBD individuals were not expected to show an effect of context. It was hypothesized that reaction times to sentences preceded by a supportive linguistic context would not be significantly faster than to those not preceded by a context.

All groups were expected to exhibit an effect of implicit causality on pronoun resolution, and thereby replicate the results of Experiment 1 . As well, in line with the findings of Pilot Studies 2a and 2b, context was not expected to modulate the influence of the implicit causality of the verbs for any of the groups tested.

Method

Subjects. The same subjects as those tested in Experiment 1 participated in this study, with the exception of LBD subject E.S. and RBD subjects F.A. and D.T. For both E.S. and F.A. the task was judged to be too difficult due to concentration difficulties. D.T. died before the study could be completed ${ }^{18}$. All of the individuals comprising the NBD control group in Experiment 1 participated in this study as well.

Materials. Apparatus and Procedure. The materials and apparatus used in this experiment were identical to those used in Pilot Study $2 \mathrm{a}^{19}$. It should be noted that the VC and VI sentences used were, in fact, the same as those used in Experiment 120. The procedure used was also identical to that used in Pilot Study 2a with two exceptions. First, as in Experiment 1, a preliminary task was presented to subjects prior to the experimental task in order to familiarize the subjects with the response procedure ${ }^{21}$. Once again it was also used to ensure that the subjects were capable of associating a particular button on the response box with a particular position. The format of the preliminary task reflected that of the experimental task. First a string of X's appeared on the screen. Subjects were instructed to press a designated button to erase the string from the screen. Once this was done an "X" and an "O" (or vice versa), appeared side by side, in the center of the computer screen. As in the preliminary task of Experiment 1, subjects were simply required to respond according to where the "O" was on the screen. Two adjacent buttons, 
other than the one used to erase the string of X's from the screen, were used for this response. If the "O" was on the left, they were instructed to press the left button of the designated pair; if it was on the right, subjects were instructed to press the right button. Reaction time and accuracy were recorded by the computer. All of the subjects included in the analyses were found to perform at a level of at least $95 \%$ accuracy on this task. All responses were made with a finger of the right hand for the controls, or of the least impaired hand for the patients 22 .

The second exception to the procedure used in Pilot Study 2a was the use of demonstration stimuli on the computer during the instructional phase of the task. As in Experiment 1, these examples were referred to when the examiner was issuing the instructions ${ }^{23}$.

Results

The experiment used a mixed design with one between groups factor and two within groups factors. The between groups factor was Group and it had three levels -RBD, LBD and NBD. The two within groups factors were Context, with two levels -context consistent (CC) and no context (NC) and Verb Consistency, also with two levels -verb consistent (VC) and verb inconsistent (VI).

As in Pilot Study 2a, filler sentences were included in the stimuli; however, only responses to the experimental sentences were analyzed. Also, as in Experiment 1, an analysis was performed which excluded the data to sentences that contained verbs to which subjects responded incorrectly on the verb test. Effects that emerged as significant by both subjects and items were the same for both sets of analyses; therefore, only the results based on the complete data set are reported.

The number of extreme reaction time values replaced in the present experiment was approximately the same across conditions and groups, ranging from $0.4-1.3 \%, 0$ $1.4 \%$, and $0-2 \%$ for the RBD, LBD, and NBD groups, respectively. As well, the number of mechanical errors and responses timed out accounted for only $3 \%, 4 \%$, and 
$0.7 \%$ of the total number of possible responses for the RBD, LBD, and NBD groups, respectively.

It should be noted that the data from LBD subject S.C. were excluded from the analyses as a result of his poor performance $(12 / 20)$ on the preliminary task. As well, based on the analysis of the accuracy data (reported below), RBD subjects J.S. and G.G. were once again found to have pressed the same button in response to all of the stimuli (with one exception for J.S.). As such, their data were also excluded from the analyses reported for this experiment. Once again, in order to remain consistent, the data for the matched controls of the RBD subjects that were excluded from the analyses - namely, G.C., Gw.B., J.C., and J.R. -- were also excluded.

Figure 9.1 shows subjects! mean reaction times to verb consistent and verb inconsistent sentences under the two context conditions for all three groups. Analysis of the data by both subjects and items revealed a significant main effect of Group $[\mathrm{E} 1(2,37)=$ $\left.9.58, \mathrm{p}<0.001 ; \mathrm{E2}(2,136)=333.58, \mathrm{D}<0.001 ; \min \mathrm{E}^{\prime}(2,39)=9.31, \mathrm{R}<0.01\right]$. Pairwise comparisons of the means using Newman-Keuls' procedure indicated that reaction times by the NBD group (mean $=5278 \mathrm{~ms}$ ) were significantly faster than those by both the LBD (mean $=9393 \mathrm{~ms}$ ) and RBD (mean $=7670 \mathrm{~ms}$ ) groups, who were not significantly different from each other, by both subjects and items $(p=0.01)$. Significant main effects were also found for Context $[\mathrm{E} 1(1,37)=37.52, \mathrm{p}<0.001 ; \mathrm{E2}(1,68)=53.91, \mathrm{e}<0.001 ; \mathrm{min}$ $\left.\mathrm{E}^{\prime}(1,84)=22.12, \mathrm{p}<0.01\right]$, with reaction times faster to $\mathrm{CC}$ than $\mathrm{NC}$ sentences, and Verb Consistency $\left[\mathrm{E} 1(1,37)=62.39, \mathrm{p}<0.001 ; \mathrm{E2}(1,68)=7.57, \mathfrak{p}<0.01 ; \min \mathrm{E}^{\prime}(1,83)=6.75\right.$, $\mathrm{p}<0.05]$, with slower reaction times to VI than VC sentences. No interactions were significant by both subjects and items.

Given that the baseline reaction times (i.e. reaction times to NC sentences) were considerably longer for the brain damaged groups compared to the NBD group, it was felt that the analysis reported above, based on absolute differences between NC and CC sentences, may not have adequately measured the effect of context. Therefore, it was 
Figure 9.1: Mean RT to VC and VI sentences according to Context BBD

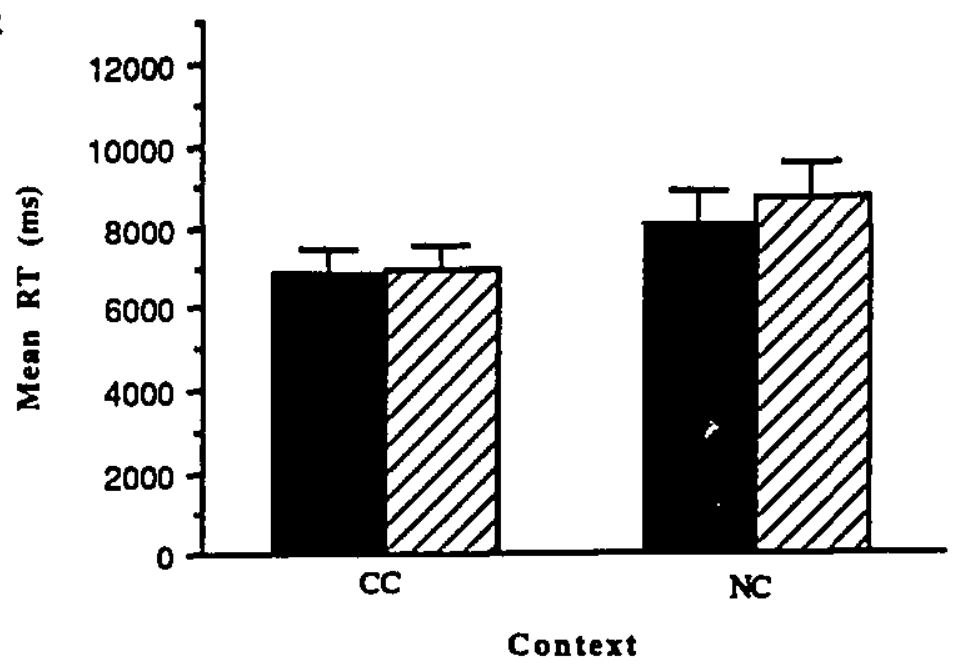

a vC

a VI

LRD

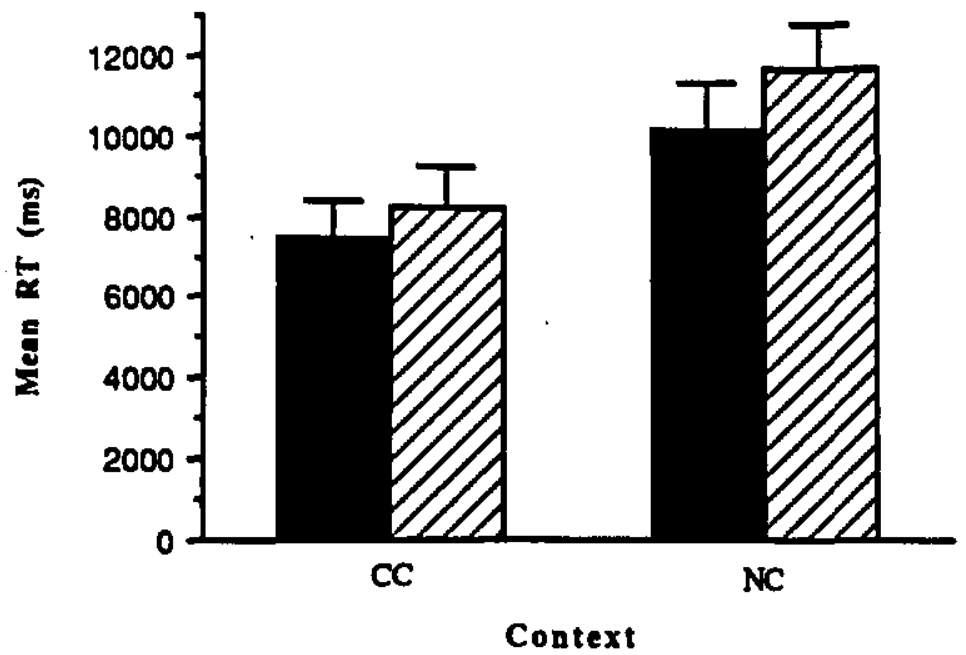

NRD

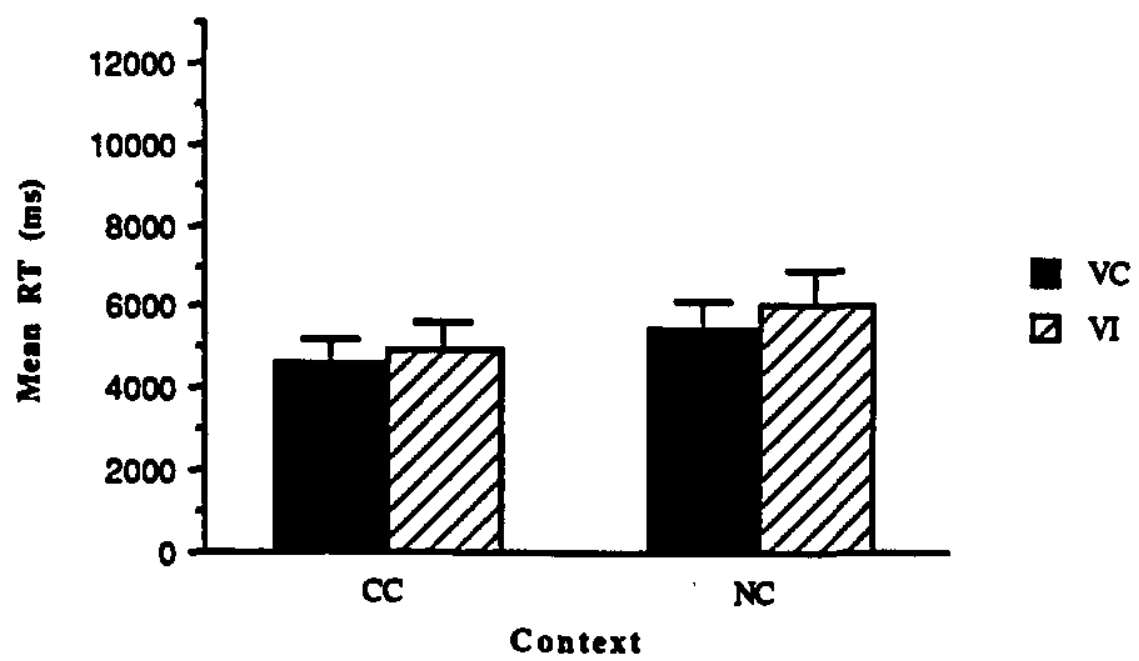


important to determine whether context effects would also emerge for each group when individual baseline reaction times were taken into account. Thus, an analysis was conducted on the differences between the mean reaction times to $\mathrm{NC}$ and $\mathrm{CC}$ sentences relative to the mean reaction times to NC sentences [i.e. (NC-CC)/NC] for each level of Verb Consistency, per subject. These new values, which represented the percentage decrease in reaction time to $\mathrm{CC}$ compared to $\mathrm{NC}$ sentences, were submitted to an analysis of variance with one between groups factor -- Group, with three levels -- LBD, RBD and NBD; and one within groups factor -- Verb Consistency, with two levels -- VC and VI. The analysis revealed a significant main effect of Verb Consistency $[E 1(1,37)=4.1$, $\mathfrak{p}=0.05]$, with a larger relative difference between mean reaction times to $\mathrm{NC}$ and $\mathrm{CC}$ sentences found for VI compared to VC sentences. Importantly, there was no effect of Group suggesting that the relative size of difference between mean reaction times to NC and $\mathrm{CC}$ sentences was consistent across the three groups.

Figure 9.2 represents the mean percentage decrease in reaction times to $\mathrm{CC}$ compared to NC sentences for the individual members of each group. A positive value indicates that responses were faster to $\mathrm{CC}$ than $\mathrm{NC}$ sentences and a negative value indicates the reverse. It is obvious from this figure that the majority of subjects from each group demonstrated a pattern of response congruent with an effect of context. In fact, all members from boh the LBD and NBD groups responded faster to CC than NC sentences for at least one level of Verb Consistency and most (LBD 8/10; NBD 13/15) for both levels of Verb Consistency. Only 2 RBD individuals (W.K. and J.R.) actually responded faster to NC than CC sentences. The others all responded faster to CC than NC sentences for at least one level of Verb Consistency with the majority (9/13) responding faster for both levels of Verb Consistency. 
Figure 9.2: Mean \% decrease in RT to CC compared to NC sentences
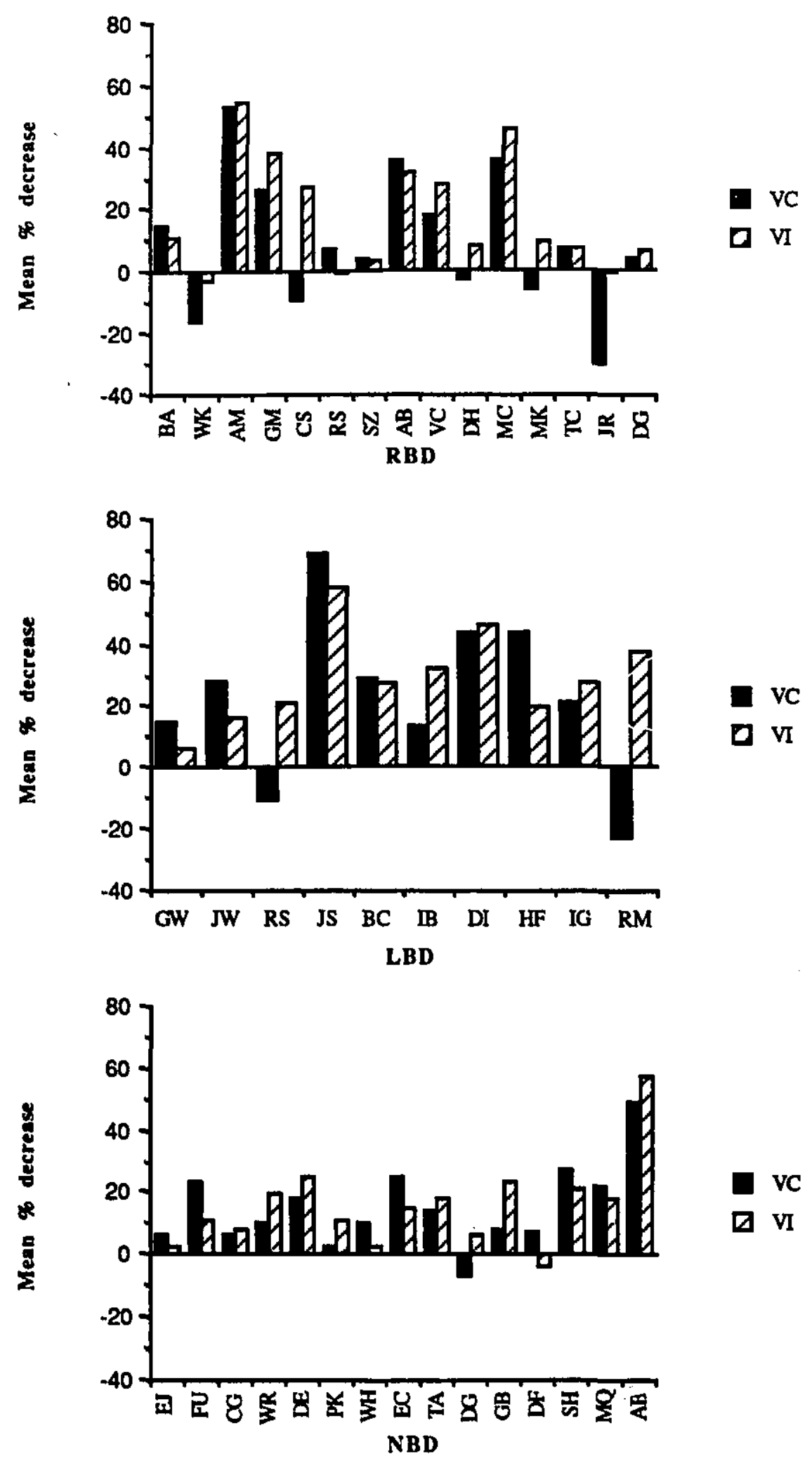
Figure 9.3: Mean $\%$ errors to VC and VI sentences according to Context BBD

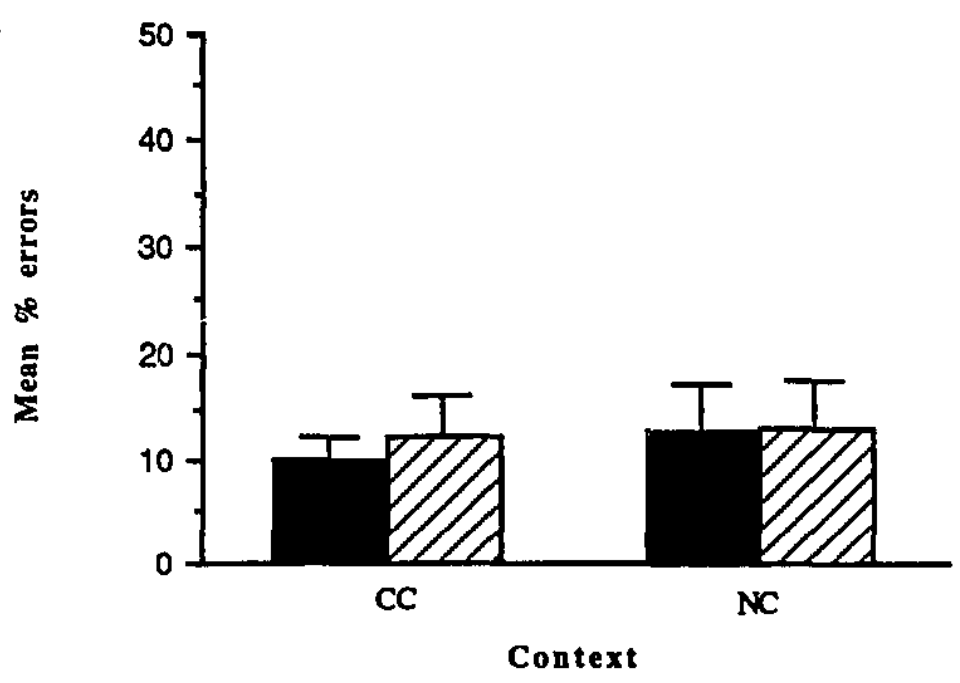

LBD

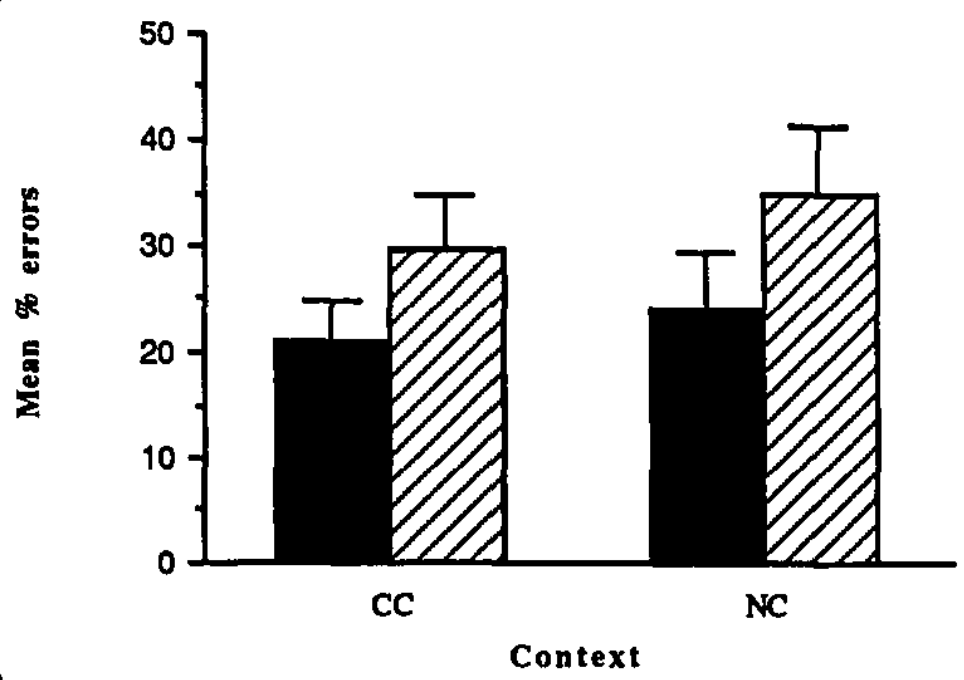

NBD

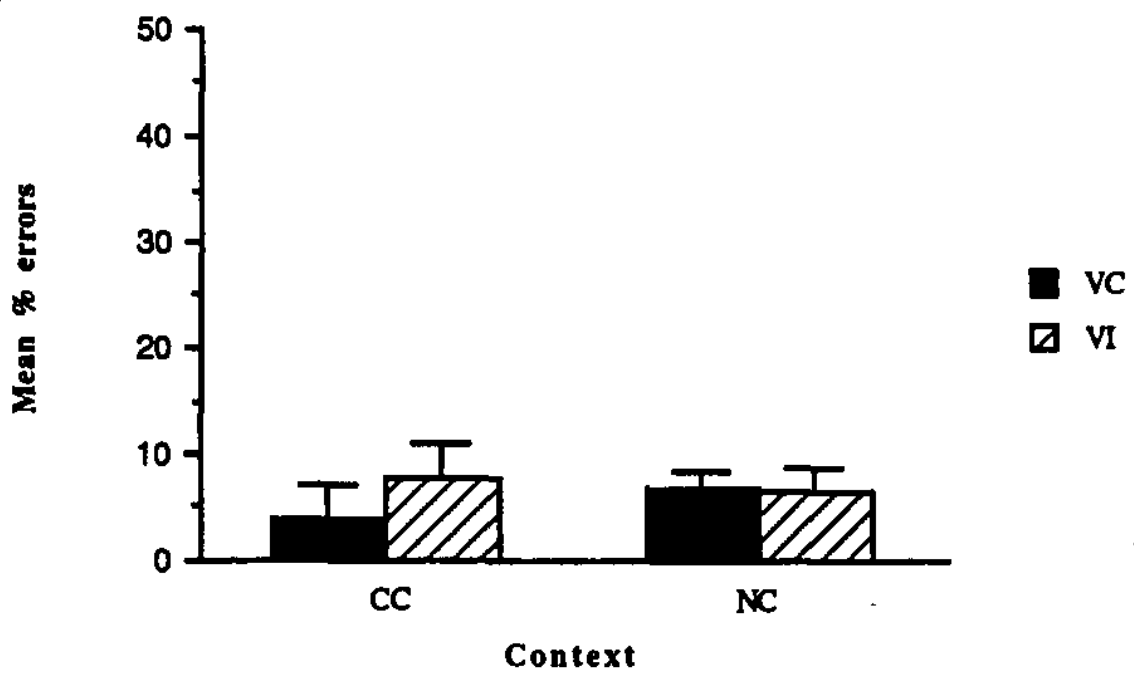


The accuracy data are represented in Figure 9.3. A significant main effect of Group $\left[\mathrm{El}(2.37)=9.98, \mathfrak{p}<0.001 ; \mathrm{E} 2(2,136)=70.76, \mathfrak{p}<0.001 ; \min \mathrm{F}^{\prime}(2,48)=8.75, \mathfrak{p}<0.01\right]$ was found. Pairwise comparison of the means using Newman-Keuls' procedure revealed that the LBD group (mean \% errors $=27$ ) made significantly more errors than both the $\mathrm{RBD}$ (mean \% errors $=12$ ) and NBD (mean \% errors $=6$ ) groups, who were not significantly different from each other, by both subjects and items $(\mathrm{p}=0.01)$. The main effect of Verb Consistency was also found to be significant $[E](1,37)=6.28, \mathrm{R}<0.05$; $\mathrm{E} 2(1,68)=10.59, \mathrm{R}<0.01]$, with more errors being made on VI than VC sentences. No interactions were found to be significant by either subjects or items.

\section{Discussion}

With respect to the reaction time data, the results of this study corroborate the findings of Pilot Studies $2 \mathrm{a}$ and $2 \mathrm{~b}$ and provide further evidence for the notion that contextual information is influential in the resolution of pronouns by NBD individuals. Responses to sentences preceded by a supportive linguistic context were found to be significantly faster than to those presented in isolation. Also consistent with the results of Pilot Studies $2 \mathrm{a}$ and $2 \mathrm{~b}$ was the finding that context could not modulate the effect of implicit causality in resolving pronouns. Reaction times to verb inconsistent sentences remained slower than to verb consistent sentences regardless of the presence or absence of a supportive preceding linguistic context.

Most important to the interests of this investigation was the finding that contextual information was also used by both groups of brain-damaged individuals in the resolution of the pronouns. Although, overall both brain-damaged groups responded more slowly than the NBD group, both LBD and RBD groups showed the same pattern of response as that of the NBD group. For both brain-damaged groups responses to sentences preceded by a supportive linguistic context were faster than to those sentences presented in isolation. Furthermore, analysis of the mean percentage decrease in reaction times to sentences preceded by a supportive context compared to those viewed in isolation indicated that the 
relative advantage gained by the use of the contextual information was consistent across groups. Additionally, inspection of individual response patterns also indicated that the use of context was a consistent pattern by the majority of individuals of all three groups, thus strengthening the interpretation of the results. Also congruent with the results of Experiment 1 was the finding that both the LBD and RBD groups showed an effect of implicit causality reflected in faster reaction times to verb consistent than verb inconsistent sentences. Similar to the pattern observed for the NBD group, the effect of implicit causality was unaffected by the presence or absence of a leading sentence, confirming the notion that this property of verbs is not affected by contextual considerations.

With respect to the accuracy data, it is interesting to note that an effect of implicit causality was also demonstrated for all groups, reflected in significantly more errors to verb inconsistent than verb consistent sentences. Note that while this finding is consistent with the those of Pilot Studies $2 a$ and $2 b$, it is in contrast to the results of Experiment 1 in which there was no effect of implicit causality based on the accuracy data. Perhaps the difference between the two sets of data lies in the fact that in this experiment all of the sentences were ambiguous. Interesting also was the finding that the factor Group did not interact with any of the other factors. Thus, similar to the results of the reaction time data, although the NBD group made significantly fewer errors than the LBD group (but not faver than the RBD group), the overall pattern of response by the brain-damaged groups tirs ife NBD control group was the same. Finally, it should be noted that there was no effect of context with respect to the accuracy data. This finding is in contrast to the results of Pilot Study 2a in which fewer errors were made to sentences preceded by a supporting context, but consistent with those of Pilot Study 2b. There is no obvious explanation for this discrepancy. However, it is worth noting that it has often been felt that given the relatively low error rates produced by NBD controls, and in this case by the RBD group as well, the interpretation of accuracy data should be made with caution. 
The overall pattem of response exhibited by the LBD group was expected. Certainly, in consideration of their linguistic deficits, it was not surprising to find the LBD group responded more slowly and made more errors than the NBD group. In addition, as noted earlier, based on the considerable evidence suggesting that LBD individuals use preceding linguistic information to compensate for language processing difficulties (Cannito et al., 1986; Friederici, 1983; Germani \& Pierce, 1992; Hough et al., 1989; Pierce \& Beekman, 1985; Pierce \& DeStefano, 1987; Pierce \& Wagner, 1985; Waller and Darley, 1978), the finding that contextual information positively influenced their ability to resolve pronouns was not surprising. Thus, the results of the present study support the current view of the use of contextual information by LBD individuals in language processing and extend it to include their use of contextual information in the resolution of pronouns.

It should be noted that there is some discrepancy between the results of the present study and those of Chapman and Ulatowska (1989). Chapman and Ulatowska (1989) found that aphasic individuals had more difficulty successfully resolving ambiguous pronouns when the resolution of the pronoun depended upon the use of preceding contextual cues as opposed to when the resolution could be made based more on the use of general world knowledge. There are three primary points to note with respect to this apparent discrepancy. First, it may indeed be the case, as the results of Chapman and Ulatowska (1989) suggest, that LBD individuals derive a greater benefit from contextual information based on general world knowledge than from that which is more specifically linguistic. However, such a claim does not discount the possibility that LBD patients may still benefit from the use of a preceding linguistic context as compared to no context at all, as suggested by the results of the present study (at least in terms of the successful resolution of pronouns).

The second point to note is that in Chapman and Ulatowska (1989), normal control subjects also had more difficulty identifying the referents of pronouns based on the use of 
preceding linguistic information as compared to instances when the use of general world knowledge was more applicable. As pointed out earlier, Chapman and Ulatowska did not include the data from both groups in the same analysis (perhaps because the error rates for the normals were quite low). However, it would have been interesting to determine whether or not the difference between the use of general world knowledge versus preceding contextual information was greater for the LBD patients than for normal controls. Perhaps LBD individuals were not specifically impaired in their ability to use the linguistic context in order to resolve pronouns when compared to normals.

A final point to note concerns the fact that in the present study successful resolution of the pronoun did not depend upon the use of the preceding context. Rather, the preceding linguistic context was merely supportive of the preferred referent. In contrast, in Chapman and Ulatowska (1989), successful resolution of the pronoun required that the preceding linguistic information be accurately processed. Thus, it may be the case with LBD patients that a supporting preceding linguistic context may be useful in processing pronouns by setting up a discourse model by which to interpret the pronoun; however, it may not be sufficient to disambiguate pronouns, without the benefit of other "extralinguistic" cues (for example, general world knowledge). Clearly, such a proposal is only speculative and needs to be further investigated.

The pattern of results exhibited by the RBD group was surprising. Although, based on the results of Experiment 1, it was not unexpected to again find an effect of implicit causality, the finding of a context effect in the resolution of the pronouns was contrary to expectations. Given the prevailing view in the literature that the underlying deficit for the observed discourse level impairments exhibited by RBD patients relates to a difficulty in using contextual information (e.g. Cook, 1989a; Gardner et al., 1983; Wapner et al., 1981), there was no apparent reason to expect the RBD group to be influenced by the information contained in the leading sentence in the resolution of the pronouns. However, as just noted, contrary to expectations, responses were faster to sentences preceded by a 
supportive linguistic context than to those presented in isolation. Furthermore, there was no significant difference between the RBD and NBD groups with respect to accuracy in assigning coreference -- thereby, suggesting comparable abilities in this area.

As was noted in the statement of the problem, while the proposal of a deficit in the use of contextual information by RBD individuals was appealing in terms of explaining a variety of seemingly unrelated discourse-level deficits, it was important to qualify the nature of this deficit. The results of the present study suggest that, at least at the level of minimal discourse and in the process of resolving pronouns, RBD patients are influenced by contextual considerations. These results, therefore, extend those of Experiment 1 and suggest that not only are RBD patients capable of integrating information between clauses in the successful resolution of pronouns, they are also able to integrate information between sentences. It is proposed that RBD individuals are similar to normals in using the information contained in the leading sentence to set up a discourse model by which to interpret subsequent utterances. Considerations as to possible reasons why RBD patients demonstrated contextual effects in performing this task will be reserved for the general discussion of this series of experiments.

To summarize, the results of this experiment were consistent with those of Pilot Studies $2 \mathrm{a}$ and $2 \mathrm{~b}$ and confirmed the notion that contextual information is influential in the resolution of pronouns. Congruent with the current view of the beneficial use of context by LBD patients in language processing was the finding that the supportive leading sentence was also influential in the resolution of pronouns by the LBD individuals. Most interesting to the purposes of this thesis, however, and in contrast to initial expectations, was the finding that RBD individuals as well derived benefit from the use of the information contained in the leading sentences in the coindexation of pronouns. Thus, these results provide evidence to suggest that RBD patients are able to use relevant information that is specifically stated in the text in the resolution of pronouns, at least at the 
level of a minimal discourse. Experiment 3 was conducted in an attempt to further define the extent of the use of contextual information by RBD individuals. 


\section{Chapter 10: Experiment 3}

The results of Experiment 2 were important in establishing a sensitivity on the part of right brain damaged individuals (as well as LBD individuals) to the contextual information specifically stated in a leading sentence to aid in the resolution of ambiguous pronouns. The purpose of Experiment 3 was to further evaluate the ability of right braindamaged patients to use and integrate contextual information from a discourse when assigning referents to pronouns. However, in contrast to Experiment 2 in which the contextual information was only supportive of a particular referent (with the resolution of the pronoun possible based solely on the information contained in the second sentence), successful resolution of the pronoun in the stimuli used in this experiment (i.e. those from Hirst and Brill, 1980) required that the contextual information be used. Furthermore, in this investigation, successful resolution of the pronoun depended upon the use of general world knowledge to determine a preferred referent based on pragmatic constraints. This is in contrast to Experiment 2 in which the supportive information was specifically stated in the leading sentence and represented, in a sense, a restatement of the disambiguating information present in the second sentence. Thus, this investigation was aimed at specifically evaluating the ability of RBD patients to use general world knowledge in relation to discursive knowledge in order to assess the plausibility of situations and, consequently, find the referent of an ambiguous pronoun. Once again, the investigation was naturally extended to a group of LBD subjects and a group of non-brain damaged controls.

Based on the results of Pilot Study 3 it was hypothesized that the NBD controls wosld show a sensitivity to the pragmatic constraints provided in the leading sentence in resolving the pronouns. Thus, reaction times to determine the pronoun in sentences with a pragmatically constrained preferred referent were expected to be faster than to sentences with no preferred referent. As well, the preferred referent, as determined by pragmatic constraints, was expected to be chosen significantly more often than the non-preferred 
referent. The LBD group was also expected to exhibit the same pattern based, once again, on the considerable evidence available suggesting that LBD patients use preceding linguistic information to aid in language processing (e.g.Cannito et al., 1986; Friederici, 1983; Germani \& Pierce, 1992; Hough et al., 1989; Pierce \& Beekman, 1985; Pierce \& DeStefano, 1987; Pierce \& Wagner, 1985; Waller and Darley, 1978)

The expectations concerning the RBD group were not so clear cut. Initial predictions were based on the prevailing view that RBD patients have difficulty using contextual information. On this view, it was expected that the RBD group would not be sensitive to the pragmatic constraints contained in the leading sentence and would, thus, have considerable difficulty disambiguating the preferred referent based on this information. However, upon consideration of the results of Experiment 2, in which RBD patients were found to use the contextual information present in the leading sentence to resolve the pronoun, there was a strong possibility that RBD patients would also be able to use the information contained in these leading sentences. However, in this experiment, in order for the information in the leading sentence to be disambiguating, the reader must successfully integrate the information between the two sentences and, more importantly, assess the plausibility of the action in the second sentence with the situation depicted in the first. Success on this task, therefore, depended to a great extent on the ability to use general world knowledge. Consequently, the possibility of a deficit on this task remained. If, however, a deficit was found, the results would be more indicative of an inability to use contextual information in light of general world knowledge and not necessarily of an absolute inability to use contextual information.

Method

Subjects. The same subjects as those tested in Experiment 2, both brain damaged and non-brain damaged, participated in this study.

Materials. Apparatus and Procedure. The matcrials, apparatus and procedure used in this experiment were all identical to those used in Pilot Study 3 with two minor 
exceptions. First, it should be noted that, as in Experiments 1 and 2, all responses were made with a finger of the right hand for the controls, or of the least impaired hand for the patients ${ }^{24}$. Second, demonstration stimuli were presented on the computer during the instructional phase of the task. As in Experiments 1 and 2, these examples were referred to when the examiner was issuing the instructions.

Results

This experiment was based on a mixed design with one between groups factor (Group) and one within groups factor (Preferred Referent). The factor Group had three levels -- right brain-damaged (RBD), left brain-damaged (LBD) and non brain-damaged (NBD) controls. The factor Preferred Referent had five levels -- NP1-Strongly Preferred, NP1-Likely Preferred, NP2-Strongly Preferred, NP2-Likely Preferred, and Neutral.

The data were examined for extreme reaction time values, but none were found for any of the groups. The number of mechanical errors and responses timed out accounted for only $2.8 \%$ and $2.0 \%$ of the total number of possible responses for the RBD and LBD groups, respectively. There were no mechanical errors nor responses timed out for the NBD group. It should be noted that the reaction time data for RBD subject M.C. (and her matched control A.B.) were excluded from the analysis because upon inspection of the accuracy data (reported below), it became obvious that M.C. almost always chose as the preferred referent the noun phrase in the first position. As a result, there were no reaction time data for items under conditions NP2-SP and NP2-LP. Data from these two subjects was, however, included in the analysis of the accuracy data as M.C.'s pattern of response was revealing in terms of strategy use (i.e. choosing the first NP as the preferred referent). It is also of interest to note that in contrast to Experiments 1 and 2, the data from RBD subjects J.S. and G.G. (and their matched controls -- Gw.B. and J.C., respectively) were included in these analyses. Interestingly, with this task, there was no strong tendency to press only one response button. As well, the data from LBD subject S.C. were also included in these analyses. 
Figure 10.1 shows subjects' mean reaction times to the various sentence types for both groups. Analysis of the data by both subjects and items revealed a significant riain effect of Group $\left[\mathrm{F} 1(2,40)=3.69, \mathrm{p}<0.05 ; \mathrm{E} 2(2,70)=40.16, \mathrm{p}<0.001 ; \min \mathrm{F}^{\prime}(2,48)=\right.$ $3.38, \mathbb{2}<0.05]$. Pairwise comparisons of the means for this factor using Newman-Keuls' procedure revealed that the LBD group responded more slowly (mean $=6026 \mathrm{~ms}$ ) than the NBD group (mean $=4078 \mathrm{~ms})$, by both subjects and items $(p=0.05)$. The mean reaction time for the RBD group ( $4890 \mathrm{~ms}$ ) was not significantly different from either of the other two groups by both subjects and items. A significant main effect of Preferred Referent was also found by subjects and marginally by items $[E 1(4,160)=14.23, \mathfrak{D}<0.001 ; E 2(4,35)=$ $2.44, p=0.065$ ]. It should be noted that given the small number of items per condition $(n=8)$ in this experiment, the power of the test using items as a random factor was extremely reduced. Pairwise comparisons of the means under the condition of Preferred Referent using the Newman-Keuls procedure revealed that responses to neutral sentences were slower than responses to all other sentence types $(\mathrm{p}=0.01)$. As well, responses to both NP2-SP and NP1-SP sentences were faster than to NP2-LP and NP1-LP $(p=0.05)$. The Group $x$ Preferred Referent interaction was not significant by either subjects or items.

A representation of the data for individual members of each group is provided in Figure 10.2. Mean reaction times to NP1-SP and NP1-LP sentences, for each subject, were combined to form one level of Preferred Referent - NP1. Similarly, mean reaction times to NP2-SP and NP2-LP sentences were combined to form a second level of Preferred Referent - NP2. As in the analysis of the complete data set, extreme reaction time values (those less than or greater than the condition mean $\pm 3 \times$ standard deviation, calculated per subject on correct responses only) were identified and replaced by that value. The number replaced was small and accounted for only $0.5 \%$ and $0.7 \%$ of all possible responses for the RBD and NBD groups, respectively. No values for the LBD group were replaced. The values depicted in Figure 10.2 represent the mean percentage decrease in reaction times to sentences with a preferred referent compared to neutral sentences. These 
Figure 10.1: Mean RT according to Preferred Referent

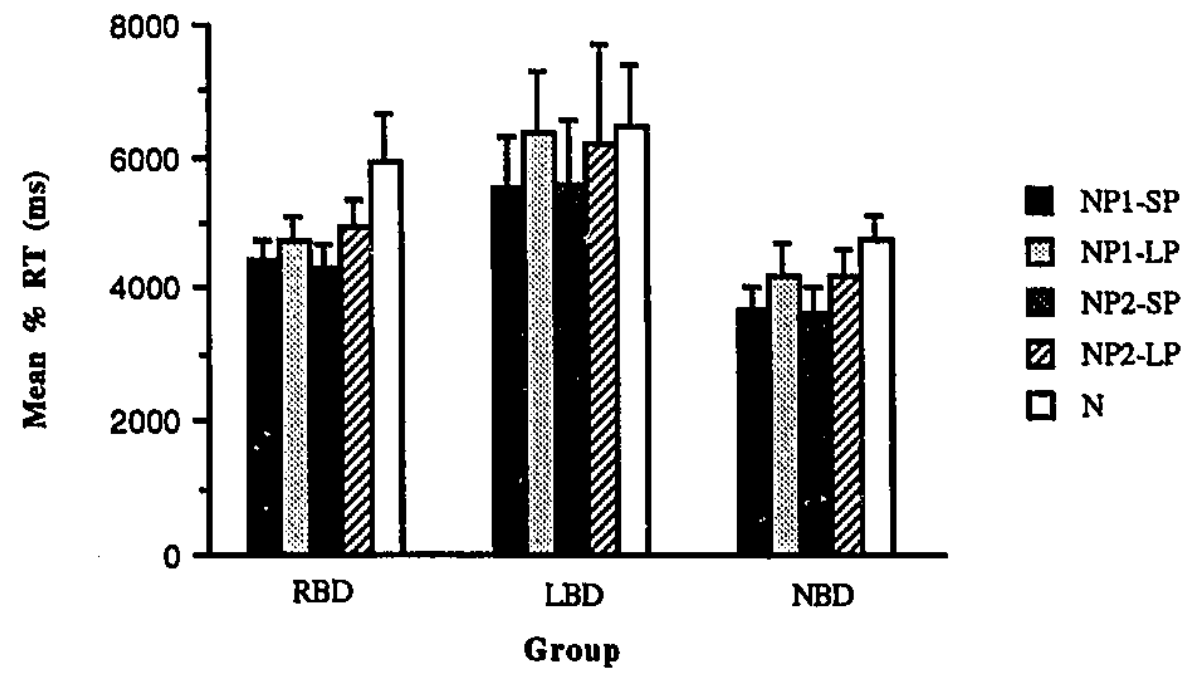


Figure 10.2: Mean \% decrease in RT to Preferred Referent vs. Neutral sentences
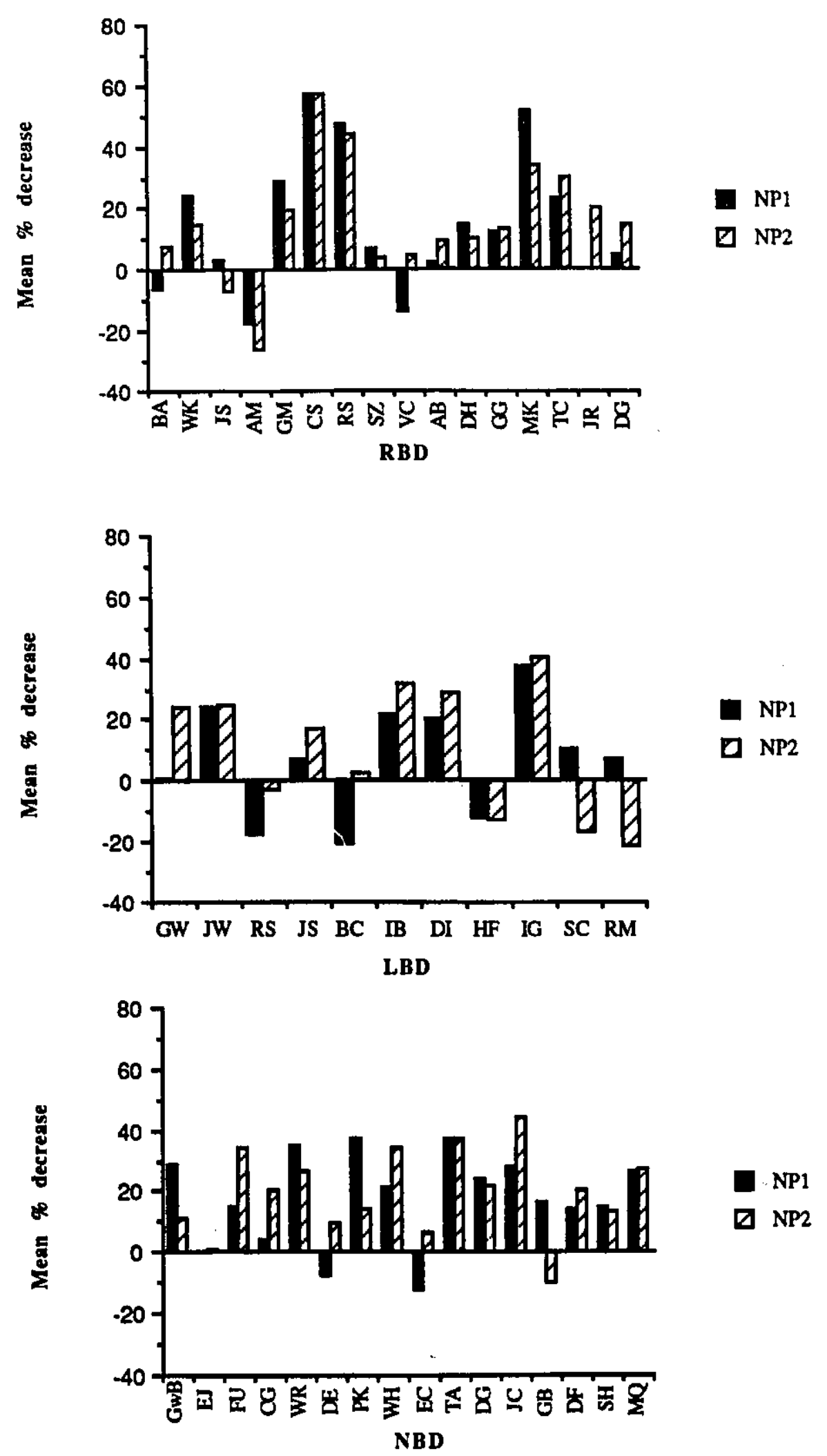
Figure 10.3: Mean \% errors according to Preferred Referent

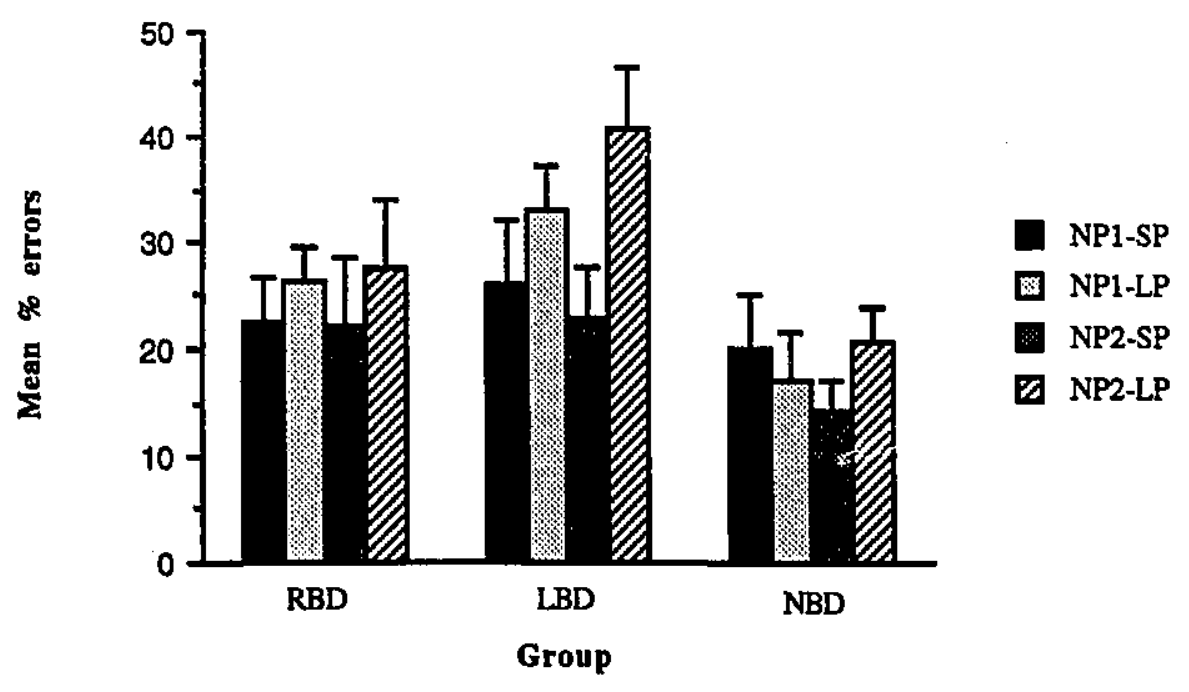


values were calculated by taking the difference in mean reaction time to Neutral and NP1 (or NP2) sentences and dividing by the mean reaction time to Neutral sentences [i.e. (Neutral-NP1 (or NP2)/Neutral]. A positive value indicates a decrease in reaction times to sentences with a preferred referent compared to neutral sentences and a negative value indicates the reverse. It is clear from Figure 10.2 that the majority of individuals from all groups responded faster to sentences with a preferred referent than to those sentences with no clearly preferred referent. Only one member of the RBD group (A.M.) and 2 members of the LBD group (R.S. and H.F.) failed to show this pattern of response for either NP1 or NP2 sentences.

The accuracy data are represented in Figure 10.3. The only effect found to be significant by both subjects and items was the main effect of Group $[E 1(2,42)=3.60$, $\mathrm{D}<0.05 ; \mathrm{E} 2(2,56)=8.33, \mathrm{D}<0.001]$. Pairwise comparisons of the group means using the Newman-Keuls procedure revealed that the LBD group (mean \% errors $=31$ ) made significantly more errors than the NBD group (mean $\%$ errors $=18$ ), by both subjects and items $(p=0.05)$. The mean percentage of errors made by the $\operatorname{RBD}$ group $(=25)$ was not significantly different from either of the other two groups by both subjects and items.

As in Pilot Study 3 and following the procedure used by Hirst and Brill (1980), average response frequencies to preferred and non-preferred referents according to preferred referent status were calculated and are presented in Table 10.1. This value represents, proportionally, the number of times a referent was chosen out of a possible 8 opportunities, based on data from all subjects.

A chi-square analysis was performed on the raw scores to compare the frequency of choice for the preferred referent versus the non-preferred referent according to associated preferred referent status for each group separately. The following results were obtained. 
Table 10.1

Average response frequencies to preferred (P) and non-preferred (NP) referents

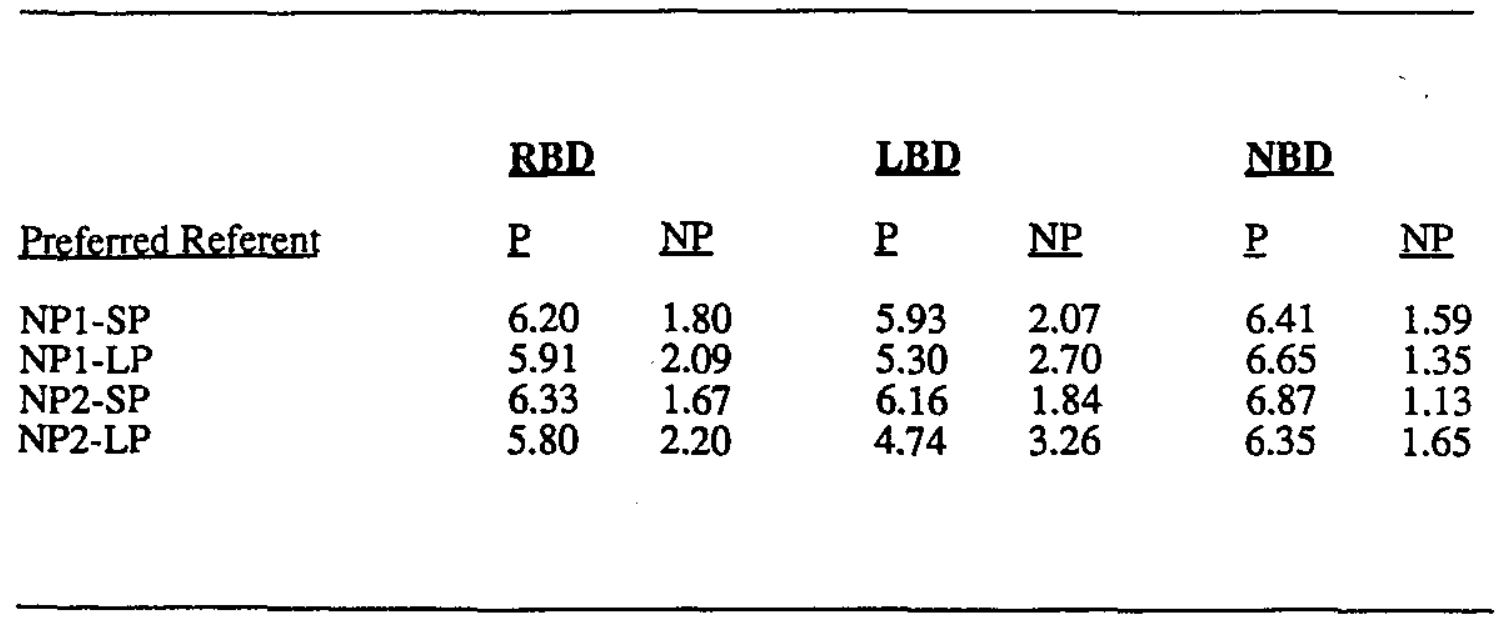


For the NBD group, the preferred referent was chosen significantly more often than the non-preferred referent for all sentence types [NP1-SP: $X^{2}(1)=49.44, \mathrm{R}<0.001$; NP1-LP: $X^{2}(1)=59.56, p<0.001 ; N P 2-S P: X^{2}(1)=70.62, p<0.001 ; N P 2-L P: X^{2}(1)=47.06$, $[2<0.001]$. Similarly, for the RBD group, the preferred referent was chosen significantly more often than the non-preferred referent for all sentence types [NP1-SP: $X^{2}(1)=40.07$, $\mathfrak{p}<0.001 ;$ NP1-LP: $X^{2}(1)=30.57, \mathfrak{p}<0.001 ;$ NP2-SP: $X^{2}(1)=45.4, \mathfrak{p}<0.001 ;$ NP2-LP: $\left.\underline{X}^{2}(1)=26.57, \underline{p}<0.001\right]$. For the LBD group, the preferred referent was chosen significantly more often for all sentence types [NP1-SP: $X^{2}(1)=19.8, \mathbb{2}<0.001$; NP1-LP: $\left.X^{2}(1)=9.12, p<0.01 ; N P 2-S P: X^{2}(1)=25.4, p<0.001\right]$ except NP2-LP $\left[X^{2}(1)=2.98\right.$, $\mathrm{p}=0.08 \mathrm{]}$. With respect to the neutral sentences, for which there was no preferred referent, choice of referent was approximately equally distributed to the first and second positions for both the RBD [1st $=4.4,2 \mathrm{nd}=3.62]$ and $\mathrm{LBD}[1 \mathrm{st}=4.05,2 \mathrm{nd}=3.95]$ groups. The NBD group demonstrated a slight, and only marginally significant tendency to prefer the referent in the first position $\left[1 \mathrm{st}=4.65,2 \mathrm{nd}=3.35 ; \mathrm{X}^{2}(1)=3.56, \mathrm{p}=0.06\right]$.

The percentage of choice of the preferred referent averaged across all sentence types for the individual members of each group is presented in Table 10.2. It is interesting to note that $10 / 17$ RBD individuals chose the preferred referent at least $75 \%$ of the time, while $14 / 17$ chose it greater than $65 \%$ of time. Only 1 member (M.C.) chose the preferred referent less than $50 \%$ of the time. These individual performances are comparable to those of the NBD group where $15 / 17$ chose the preferred referent at least $75 \%$ of the time and 1 member (A.B.) actually chose the preferred referent less than $50 \%$ of the time. Not surprisingly, values for the members of the LBD group are somewhat reduced in comparison to the RBD and NBD groups. While the preferred referent was chosen at least $75 \%$ of the time by $5 / 11$ members and greater than $65 \%$ of the time by $6 / 11$ members, five of the members chose the preferred referent less than $65 \%$ of the time. 
Table 10.2

Percentage choice of preferred referent averaged across NP1 and NP2 sentences

RBD

LBD

NBD

B.A. 66

G.W. 88

Gw.B. 88

W.K. 66

J.W. 63

E.J. 66

J.S. $\quad 69$

R.S 75

F.U. $\quad 91$

A.M. 59

J.S. $\quad 56$

C.G. 81

G.M. 81

B.C. 77

W.R. 91

C.S. 88

I.B. $\quad 77$

D.E. 84

R.S. $\quad 77$

D.I. 91

P.K. 81

S.Z. $\quad 72$

H.F. 53

W.H. 94

V.C. 75

I.G. 63

E.C. 88

A.B. 56

S.C. 70

T.A. 88

D.H. 78

R.M. 50

D.G. 78

G.G. 81

J.C. 88

M.C. 42

G.B. 97

M.K. 87

D.F. 75

T.C. 97

S.H. 81

J.R. $\quad 94$

M.Q. 84

D.G. 94

A.B. 44 


\section{Discussion}

The results of this study support those of Pilot Study 3 and corroborate the finding by Hirst and Brill (1980) that contextual information is used to constrain pragmatic choices of antecedents by NBD adults. When the preferred referent was chosen, reaction times to those sentences which biased a particular referent based on general world knowledge were faster than to sentences for which there was no preferred referent. Once again, more interesting to the purposes of this study, was the finding that both brain-damaged groups were also sensitive to bias imputed by the pragmatic information contained in the leading sentence. Thus, although NBD individuals were found to respond faster than LBD patients (although not significantly faster than RBD patients), both brain-damaged groups exhibited the normal pattern of response by responding more quickly to sentences which biased a preferred referent. The strength of this effect is seen in the inspection of the individual data which reveals that only 1 RBD patient and 2 LBD patients failed to demonstrate this pattern of response for at least one sentence type (NP1 or NP2 preferred referent).

Based on the analysis of variance of the accuracy data, the only significant finding was that LBD individuals made considerably more errors than NBD controls. Once again, as in Experiments 1 and 2, there was no difference between the NBD and RBD groups in terms of the successful resolution of the pronoun.

The analysis of the data corresponding to the frequency of choice of the preferred referent was more informative with respect to the abilities of each group to choose the preferred referent based on the pragmatic constraints provided in the leading sentence. All three groups were found to choose the preferred referent significantly more often than the non-preferred referent. The only exception to this were subjects in the LBD group who were not as sensitive to the information provided in sentences which only weakly favored the second noun phrase as the preferred referent. Thus, although the LBD group showed a sensitivity to the pragmatic information contained in the leading sentence, it was not as great as that exhibited by both the NBD and RBD groups. This pattern of performance was 
also reflected in the analysis of the individual data. While the rnajority of members of both the RBD and NBD groups chose the preferred referent at least 65\% of the time (and most of those at least $75 \%$ of the time), nearly half of the LBD subjects chose the preferred referent less than $65 \%$ of the time.

The pattern of response exhibited by the LBD group was primarily as expected. Once again, LBD patients were found to use the contextual information present in the leading sentence to aid in the resolution of ambiguous pronouns. This finding supports the results of Experiment 2 and extends it to suggest that not only are LBD individuals influenced by the presence of a supporting context (as in Experiment 2), they are able to use the information in a preceding context to pragmatically constrain antecedent choices based on general world knowledge. It should be noted that the finding of the use of pragmatic constraints by LBD individuals in language processing is consistent with the results of others (e.g. Caramazza \& Zurif, 1976; Deloche \& Seron, 1981; Heeschen, 1980; Kudo, 1984) who have found improved sentence processing by LBD patients when interpretation is constrained by the plausibility of the events depicted (for example, in semantically constrained nonreversible sentences [The apple that the boy is eating is red]). Returning to the results of the present study, it was not surprising to find, however, that LBD individuals were still not as successful as NBD individuals in assigning coreference, reflected in a greater overall error rate. The important point to emphasize, however, is that as a group they did show some sensitivity to the pragmatic information contained in the leading sentence and most were able to respond according to these constraints in successfully resolving ambiguous pronouns with greater than chance accuracy.

The RBD group performed in accordance with the results of Experiment 2. Contrary to initial expectations, but consistent with the findings of Experiment 2, the RBD group demonstrated an effect of context similar to that exhibited by the NBD and LBD groups. They demonstrated a sensitivity to the pragmatic information contained in the leading sentence and evidenced an ability to integrate the information between sentences in 
order to constrain the choice of a preferred antecedent based on general world knowledge. Thus, they responded faster to sentences in which there was a clearly preferred referent than to those in which there was not a preferred referent; and, they chose the referent biased by the information in the context (the preferred referent) significantly more often than the non-preferred referent. As well, unlike the LBD group, there was no significant difference with respect to overall error rates between the RBD and NBD group. Once again, therefore, the RBD patients demonstrated an ability to assign coreference comparable to NBD controls.

It is of interest to note that these findings somewhat contrast those of Brownell et al. (1992), who found that RBD patients favored coindexation based on linguistic factors irrespective of the plausibility of the resultant interpretation. Their results thus suggested a reduced sensitivity to plausibility constraints imposed by the use of general world knowledge. However, in the Brownell et al. (1992) study, the demands of the task were considerably different from those of the task used in Experiment 3. Subjects were required to judge the appropriateness of a response to a question set up by a previous discourse. Pragmatic information was not involved in constraining the choice of an antecedent. Rather, the pragmatic information was involved in the determination of a response as appropriate or "something else" (such as sarcastic or joking). The results of Brownell et al. (1992), therefore, suggested that RBD patients relied to a greater extent than normals on the use of pronouns in establishing the coherence of a discourse. Thus, when a pronoun was present, they were not as successful as normals at judging an implausible response as being, for example, sarcastic. The results of Brownell et al. (1992) are, therefore, more suggestive of difficulty in using general world knowledge to override an interpretation that was established based on linguistic factors, such as coherence as a function of coreference. The results of the present experiment, however, suggest that RBD patients are not completely insensitive to pragmatic constraints, and are certainly capable of using such information to assign coreference. 
Overall, the results of the present experiment corroborate the findings by Hirst and Brill (1980) that contextual information is useful in constraining antecedent choices of ambiguous pronouns by NBD adults. More interestingly, these results corroborate the findings of Experiment 2 and demonstrate that both LBD and RBD individuals are also able to use contextual information in the resolution of pronouns. Thus, these results provide further support for the view that RBD individuals are influenced by the use of contextual information at the level of a minimal discourse to resolve ambiguous pronouns. Furthermore, the results of this study extend this view to suggest that not only are RBD (and LBD) patients influenced by a supportive linguistic context, but also that they can actively use contextual information in light of general world knowledge to constrain antecedent choices. 


\section{Chapter 11: General discussion for Experiments 1, 2, and 3}

Based on the current view that difficulties in the use of contextual information underlie many of the discourse level deficits exhibited by RBD individuals, three experiments were conducted with the goal of more clearly defining the nature of this proposed impairment. The three experiments sought to focus on the use of contextual information at lower levels of processing than usually investigated with this population. Thus, in Experiment 1, the use of contextual information at the level of the single sentence, through the integration of information between clauses, was investigated. Experiments 2 and 3 focused on the use of contextual information across the sentence boundary, at the level of a minimal discourse (i.e. two sentences). In all three experiments the resolution of pronouns was used as the linguistic vehicle by which to investigate the ability of RBD individuals to use context. This linguistic process was chosen as the target of the investigation for two main reasons. First, given its important role in discourse processing in terms of promoting cohesion and coherence, and the view that RBD individuals have difficulty in many areas of discourse processing, the ability to resolve pronouns by RBD patients seemed a natural candidate for study. Second, it was thought that pronoun resolution by $R B D$ individuals would be an interesting area of study because the process of resolving pronouns has been shown to be influenced by both linguistic and contextual factors. The investigation was naturally extended to a group of LBD individuals and a group of NBD controls.

The NBD group performed as expected. In Experiment 1 they evidenced an effect of implicit causality in terms of responding more slowly to sentences that established an interpretation that was inconsistent rather than consistent with a verb's natural bias, even when the pronoun was unambiguous based on gender cues. Thus, these results provided more evidence for the robustness of the effect of implicit causality and supported the view that contextual information following a pronoun is influential in ultimately determining 
coreference. The results for the NBD subjects in both Experiments 2 and 3 were consistent with the findings of the Pilot Studies 2a, 2b and 3. In Experiment 2, the resolution of pronouns in sentences preceded by a leading sentence was faster than to those sentences presented in isolation. In addition, it was found that the information contained in the leading sentence was not capable of modulating the effect of the implicit causality of the verb. Once again, therefore, these results were consistent with a modular view of language (Fodor, 1983; Forster, 1979), in which context exerts its influence, following initial firstpass processing. As well, these results supported the notion of the contextual impenetrability of verbs (Shapiro et al., 1987; 1989). Recall that Shapiro et al. (1987; 1989) found evidence to suggest that during sentence processing all of the potential argument structures associated with a verb are activated, regardless of the biasing information contained in the preceding context. The results of Pilot Studies $2 \mathrm{a}$ and $2 \mathrm{~b}$ and Experiment 2 were consistent with the view that the processing of verbs is resistant to contextual forces by demonstrating that the property of verbs termed implicit causality, is also not modulated by contextual factors. The results of Experiment 3 replicated the findings of Hirst and Brill (1980) and Pilot Study 3 in demonstrating that the resolution of pronouns is subject to pragmatic constraints based on general world knowledge.

The pattern of performance exhibited by the LBD group was also primarily as expected. In Experiment 1, the LBD group demonstrated a sensitivity to the implicit causality of verbs as evidenced by slower reaction times to verb inconsistent than verb consistent sentences, corroborating the results of Grober and Kellar (1981) who also found that a sensitivity to this property of verbs was preserved in LBD individuals. The results of Grober and Kellar (1981), however, also suggested that LBD patients may have difficulty integrating information between clauses, reflected in close to chance responding to ambiguous verb inconsistent sentences. In contrast to this finding, the results of Experiment 1 suggested that LBD individuals can, in fact, integrate information between clauses in order to resolve pronouns, albeit not as successfully as NBD individuals. 
The results of both Experiments 2 and 3, in which LBD individuals demonstrated an effect of context similar to the NBD group, were expected and consistent with the overwhelming evidence supporting the view that LBD individuals use contextual information to help compensate for language processing difficulties (e.g.Cannito et al., 1986; Friederici, 1983; Germani \& Pierce, 1992; Hough et al., 1989; Pierce \& Beekman, 1985; Pierce \& DeStefano, 1987; Pierce \& Wagner, 1985; Waller and Darley, 1978). These results extended that view to include the use of contextual information in the resolution of ambiguous pronouns. Moreover, the results of Experiment 3 suggested that LBD patients could actively use contextual information to pragmatically constrain antecedent choices.

The pattern of performance exhibited by the RBD group was the least expected and certainly the most interesting for the primary purpose of this thesis. The most striking finding, which was consistent across the three experiments, and demonstrated by the majority of individual patients, was that the RBD group was as successful as the NBD control group in using contextual information to resolve pronouns. This finding was true both at the level of the single sentence, which focused on the integration of information between clauses and at the level of a minimal discourse (i.e. two sentences). Thus, in Experiment 1, the RBD group was similar to both the NBD and LBD groups in dr monstrating an effect of implicit causality in terms of faster reaction times to verb consistent than verb inconsistent sentences for both ambiguous and unambiguous sentences. The preservation of a sensitivity to this property was not entirely unexpected given that for the most part syntactic and semantic abilities are preserved in RBD patients. However, with respect to the RBD group, it was important to demonstrate this effect in terms of reaction times. It was argued that in order to respond to the inconsistency of the interpretation provided by the subordinate clause of the sentence to the verb's natural bias, in terms of a slower reaction time but subsequent ability to successfully resolve the pronoun, integration of the information between the clauses must be made. Therefore, the 
demonstration of an effect of implicit causality based on the reaction time aata provided strong evidence for the notion that RBD patients are able to use contextual information at the level of the single sentence, in terms of the integration of information between clauses. This was an important finding, given the goal of defining a proposed impairment in the use of contextual information in terms of different levels of language. Another interesting finding that emerged from the results of this experiment concerned the claim that RBD individuals have difficulty revising initial interpretations (e.g. Brownell et al., 1986; Schneiderman \& Saddy, 1988; Ulatowska \& Baker, 1976). In fact, the results of this experiment suggested otherwise, at least with respect to the factor of implicit causality. It was argued that in order to successfully resolve the pronouns in the verb inconsistent sentences, the initially preferred referent, as determined by the bias imputed by the verb, must have been discarded in favor of the one which best fit semantically with the interpretation set up by the final clause of the sentence.

Experiments 2 and 3 addressed the use of contextual information by RBD individuals across sentence boundaries. Contrary to initial predictions, the RBD group was found to use the contextual information in a manner similar to normals in order to resolve the pronouns. In Experiment 2, the supportive linguistic context resulted in faster reaction times to determine preferred referents. These results suggested that the RBD patients were using the information contained in the leading sentence to set up a discourse model by which to interpret subsequent pronouns. It was also of interest to find that not only did the RBD patients demonstrate a sensitivity to the preceding contextual information in terms of reaction times, they were as successful as the NBD controls in resolving the pronouns.

The task demands of Experiment 3, in terms of the influence of contextual information were slightly different than those of Experiment 2. In Experiment 3, the use of the preceding linguistic context was mandatory for the successful resolution of the pronoun. Moreover, while in Experiment 2, the relevant contextual information was specifically stated in the leading sentence and was, for the most part, a restatement of the 
disambiguating information in the pronominal sentence, the successful use of the contextual information in Experiment 3 required the use of general word knowledge. In spite of these different demands, RBD patients once again evidenced a strong effect of context. Their pattern of performance was virtually indistinguishable from that of NBD controls. These results suggested that not only are RBD patients influenced by contextual information in terms of supporting preferred referents (Experiment 2), they are capable of invoking its use to constrain pragmatically determined preferred referents. Thus, RBD patients demonstrated the normal pattern of response in reacting to the pragmatic constraints derived by integrating the information contained in the leading sentence with that contained in the pronominal sentence and interpreting this in light of general world knowledge. It is interesting to note that the results of Experiment 3 may be interpreted as counter-evidence to the proposal by Gardner et al. (1983) and Wapner et al. (1981) that some of the narrative deficits exhibited by RBD patients relate to difficulty in using their "plausibility metric". Gardner et al. (1983) and Wapner et al. (1981) have suggested that RBD patients are impaired in their assessment of the plausibility of certain events relative to information contained in the whole narrative. The results of Experiment 3 of the present investigation, however, demonstrated that RBD patients were, in fact, capable of making (non-overt) plausibility judgments, at least at the level of a minimal discourse. Therefore, it appears that the suggestion of an overall deficit in the ability of RBD individuals to assess the plausibility of events is simply incorrect.

To summarize, the results of Experiments 1, 2, and 3 provided strong evidence to suggest that RBD patients are sensitive to the influence of contextual information in resolving pronouns at the level of the single sentence, in terms of the integration of information between clauses, and at the level of a minimal discourse. An obvious question that emerges is why did these results obtain. Given the considerable evidence, based on investigations of a number of different discourse level phenomena, that has suggested that RBD patients have difficulty integrating contextual information in the processing of 
language (e.g. Gardner et al., 1983; Schneiderman et al., 1992; Wapner et al., 1981; Weylman et al., 1989), there was no apparent reason to expect that they would benefit from the use of a preceding linguistic context in the resolution of ambiguous pronouns.

One possible explanation to account for the discrepancy in results between the present investigation and those of studies that arc suggestive of an impairment in the use of contextual information by RBD patients relates to the variability among patients tested. Certainly, it is possible that there exists a subset of RBD individuals who have difficulty using contextual information and that the patients of the present investigation simply did not represent this particular subset. A potential factor that might help to discriminate between such individuals concerns the severity of the brain damage. Unfortunately, it is not possible to directly compare the severity of the patients tested in the present investigation with those of other studies. In the present study, a formal measure of cognitive abilities of the subjects in each group was not collected, and this is also true of many other studies. However, it should be noted that the subjects of the present investigation were likely quite high functioning, as necessitated by the demands of the task. On the other hand, it can also be argued that this is likely the case for subjects in other studies as well, since the tasks are often quite challenging linguistically. Nonetheless, to the extent that there is variability among individual $R B D$ patients with respect to severity of brain damage and accompanying cognitive deficits, it is possible that impaired use of contextual information by some RBD patients may be a function of impaired cognitive skills. In fact, the possibility of cognitive deficits accounting for impaired language processing by RBD patients is not novel and was proposed by Archibald and Wepman (1968) to account for the linguistic deficits they identified in some of their RBD patients. Thus, the finding in the present investigation of the ability of RBD individuals to use contextual information in the processing of ambiguous pronouns may be reflective of only mild to moderate brain-damage and relatively intact general cognitive abilities. 
On the other hand, it should be noted that this explanation is not completely satisfactory when one considers the results of the individual analyses for all three experiments of the present investigation. The size of the RBD group used in this investigation was relatively large. As well, the group was representative of a number of different lesion sites. Given the size of the group and the varying lesion sites, it may be argued that there existed a certain amount of natural variability among the RBD patients tested. In fact, it was this expectation that prompted the investigation of individual patient response patterns. However, irrespective of this variability, one of the most striking findings of this investigation was the consistency of response patterns across individual patients. As previously noted, the finding of a pattern of response reflective of the use of contextual information was characteristic of the majority of RBD individuals tested. In addition, it was interesting to find upon closer inspection of the data, that of the RBD patients who failed to show a pattern of response suggestive of the use of contextual information in any of the three experiments, only one (A.M.) was found to exhibit this pattern of response in more than one experiment. Moreover, she failed to show the pattern of response in Experiments 1 and 3, suggesting that her problem was not necessarily related to specific difficulty in using contextual information across sentences (as Experiment 1 tested the ability to use contextual information at the level of the single sentence). Thus, while one can certainly not discount the possibility that the discrepancy between the results of this investigation and those of studies that have found deficits in the use of contextual information by RBD patients is a function of patient variability, it does not appear to be the most satisfactory explanation of the present results.

Another possible explanation to account for the finding of context effects by RBD individuals in the present investigation is found in consideration of some recent evidence. As was discussed in the statement of the problem, while the suggestion of a deficit at the level of contextual integration was appealing in terms of explaining a number of seemingly varied discourse level deficits exhibited by RBD individuals (e.g. processing of humor, 
indirect speech acts, non-literal language, etc.), direct tests of this hypothesis were lacking. In fact, a few studies that specifically manipulated the factor of context (Tompkins, 1990; Tompkins, 1991; Tompkins \& Flowers, 1987) did find evidence to suggest that RBD individuals can use contextual information, subject to certain factors. These factors, clearly detailed by Tompkins and her colleagues, appear relevant to the interpretation of the results of the present investigation.

A recurrent theme in many of Tompkins' papers (Tompkins, 1990; Tompkins, 1991; Tompkins et al., 1992; Tompkins \& Flowers, 1987) concerns the distinction between "automatic" and "effortful" processing. She notes (based on the work of Hasher and Zacks (1979) and Posner and Snyder (1975), among others) that automatic processing is not subject to an individual's intentions nor, importantly, is it thought to be a function of attentional constraints. Thus, automatic processing is thought to proceed relatively freely at a less conscious level of cognition. In contrast, it is believed that effortful processing is guided by intentions and proceeds at a much more conscious level. Tompkins (1990; Tompkins et al., 1992; Tompkins and Flowers, 1987) has argued that many of the tasks used in studies which have identified a problem in the use of contextual information by RBD individuals have necessitated effortful processing. She highlights, for instance, story retelling tasks and the inferencing of morals as in Wapner et al. (1981) as examples of tasks requiring effortful processing. Indeed, many other such tasks come to mind upon consideration of the literature. For instance, consider the efforful processing requireci to impose the use of contextual information derived from a theme setting sentence on the arrangement of sentences into coherent stories such as in Schneiderman et al. (1992). Similarly, the choice of congruent punch-lines to jokes (e.g. Brownell et al., 1983; Wapner et al., 1981) or appropriate continuations of preceding paragraphs favoring a literal or indirect interpretation of a request (Weylman et at., 1989) seemingly require a more conscious manipulation of relevant information. 
Tompkins (1990) has further proposed that reduced abilities to engage in more effortful processing by RBD individuals may be a function of impaired attentional capacities. She cites evidence of the relationship between the right hemisphere and "attentional arousal" (e.g. Coslett, Bowers, \& Heilman, 1987) and suggests that perhaps RBD individuals are more vulnerable to reduced attentional states. She argues, therefore. that a reduction in attentional resources makes effortful processing, involving strategy development for the completion of tasks, difficult. The difficulty entails not having enough resources to. share with both the act of engaging in the task and the generation of efficient strategies to complete the task. With respect to difficulty in the use of contextual information, Tompkins (1990) has proposed, based on the view of Craik and Byrd (1982), that effective conscious encoding of specifically relevant information may be impaired due to a reduced attentional state in RBD individuals; however, generalities of meaning may be adequately encoded. Thus, she states, "Inaccurate inferences or failure to integrate all sources of information to resolve indeterminacies of meaning (Burgess \& Simpson, 1988) may reflect the products of impoverished encoding, and/or may occur because insufficient resources are available to operate on encoded information" (p. 315).

Tompkins and her colleagues have tested the distinction between automatic and effortful processing and its effect on the processing of language by RBD individuals with interesting results. Tompkins (1990), for example, has demonstrated that, contrary to the prevailing view that RBD patients are impaired in their knowledge of metaphoric language, this knowledge appears to be intact when elicited through the use of more automatic processing. In a priming experiment using an auditory lexical decision task that favored automatic processing (through, among other things, the use of a short interval between the prime and the target) it was found that reaction times to target words that were preceded by primes related to the metaphorical meaning of a target were shorter than to target words preceded by a neutral prime. This pattern of response was similar to that exhibited by the normal controls. Similarly, Tompkins et al. (1992) found that knowledge of idiomatic 
expressions was intact in RBD individuals when the knowledge of such expressions was tapped through the use of a word-monitoring task (a task that presumably favors automatic processing). In contrast, when RBD patients were required to provide the definition of an idiom (a more effortful task), they evidenced considerably more difficulty than did normals and, consequently, reflected the more prevalent view that $\mathrm{RBD}$ individuals are impaired in their processing of idiomatic expressions.

As an aside, it is interesting to note that the results of Tompkins and her colleagues (Tompkins, 1990; Tompkins et al., 1992) may also be viewed in terms of the distinction between on-line versus off-line tasks. In fact, Tompkins (1992) actually makes this distinction. To the extent that on-line tasks reflect initial, first pass-processing and, hence, are thought to proceed relatively involuntarily (Fodor, 1983) and that off-line tasks favor second-pass processing which is believed to be more strategic, the association of on-line tasks with automatic processing and off-line tasks with more effortful processing is easily made. However, the question becomes whether or not there is a direct one-to-one mapping of on-line tasks with automatic processing and off-line task with effortful processing; or instead, for example, can some off-line tasks entail more automatic processing? Although there is no empirically obvious answer to this question, as will be seen in the discussion of the results of the present investigation, there is reason to believe that the mapping of on-line tasks with automatic processing and off-line tasks with effortful processing, is not necessarily mutually exclusive. It will be argued that certain aspects of language processing (e.g. pronoun resolution) are naturally more automatic, regardless of the task used to investigate the processing involved. To this end, therefore, it is believed that the explanation of language processing difficulties exhibited by RBD patients in terms of the distinction between automatic and effortful processing is preferable to that based on the distinction between on-line versus off-line tasks.

Returning to the argument that some apparent language difficulties exhibited by RBD patients may be a function of effortful processing, it is interesting to find that some 
studies (Glosser \& Goodglass, 1991; Joanette et al., 1988) investigating lexical-semantics in RBD individuals have also invoked the notion of automatic versus "controlled" or effortful processing in the explanation of their results. Joanette et al. (1988), for example, investigated the time course of word-naming by RBD patients. They found that RBD individuals did not differ from normal controls, in terms of the number of items named, in the first 30 seconds of the naming period. After this period, however, RBD patients were found to produce significantly fewer items than the normals. The authors suggested, in accordance with the view by Rosen (1980, cited in Joanette et al., 1988)) that the processes involved in a word-naming task are different at the beginning of the task than later on, that the high production of words in the first 30 seconds probably reflected an automatic period of lexical access. Reduced production after the initial period of automatic processing, on the other hand, was likely a reflection of a reduced ability to effectively organize retrieval strategies for more lexical items -- obviously a more effortful processing requirement. Along a similar line of reasoning, Glosser and Goodglass (1991) have proposed that the idiosyncratic word associations produced by some RBD individuals in a word association task reflect a disruption in "controlled" processing. They found that although RBD individuals were able to successfully produce word associations that were similar to those produced by normals, there were instances when their responses were odd and idiosyncratic (e.g. asssociating "snake" with "memory"). The authors noted that during the instances when idiosyncratic word associations emerged, response times were increased. They thus reasoned that idiosyncratic responses come about from a failure to initially associate a word of high frequency to the target word. This results, consequently, in the initiation of a more effortful search. The authors proposed, therefore, that it is in the effortul search for an associate that RBD patients are impaired.

To summarize, there is some recent evidence to suggest that the distinction between automatic and effortful processing is a useful one to make when considering various language deficits exhibited by RBD patients. The emerging view is that right brain damage 
may result in reduced processing resources and may consequently impair the ability to engage effectively in effortful processing while automatic processing remains relatively intact. There are two main points to note with respect to this claim. First, it is important to emphasize that this claim does not necessarily imply that effortful processing is the exclusive domain of the right hemisphere. In fact, it has recently been proposed that some language deficits exhibited by LBD individuals may also be a function of reduced processing resources (McNeil \& Kimelman, 1986; McNeil, Odell, \& Tseng, 1991). The results of Tyler (1985) may be viewed as supporting such a view. She found that an agrammatic patient demonstrated a sensitivity to verb subcategorization restrictions in a word-monitoring task, reflected in increased reaction times to sentences which violated these restrictions (e.g. monitoring for the word "guitar" in "The young man slept the guitar"). However, the same patient exhibited a reduced ability, as compared to normals, to explicitly judge whether or not a sentence such as that presented in the example above was anomalous. One might propose, therefore, that the discrepancy in abilities between the two tasks can be explained in terms of different processing demands. Thus, while the patient demonstrated knowledge of subcategorization restrictions at a relatively unconscious level of processing (as in the word-monitoring task), he had difficulty in actively using this knowledge in the more effortful task of judging sentence acceptability.

Returning to the proposal that right brain damage also affects the ability of an individual to engage in effortful processing, it appears, therefore, that a key distinction concerns the level of language at which deficits related to effortful processing appear as a function of left or right brain-damage. Given the typical language profiles of left and right brain-damaged individuals, it seems obvious that different levels of language are selectively vulnerable to increased processing demands as a function of left versus right brain damage. This brings us to the second point which is that, with respect to rignt brain-damage, evidence suggests that reduced processing resources and concomitant difficulties in effortful processing, exert their influence at higher levels of language processing -- more 
specifically, at the level of discourse (although the results of Joanette et al. (1988) and Glosser and Goodglass (1991) also suggest some influence at the level of semantics). Thus, some apparent linguistic deficits associated with difficulty in using contextual information (such as difficulty in the interpretation of indirect speech acts, or in arranging sentences into coherent stories) evidenced by RBD individuals may in fact be more of a reflection of impaired processing related to task demands, rather than absolute deficits in the ability to use contextual information.

With respect to the results of the present investigation, an interesting explanation concerning why RBD patients evidenced an ability to use contextual information surfaces in relation to the distinction between automatic and efforful processing. It can be argued that the use of contextual information in the completion of the tasks used in this study was a function of more automatic processing. Granted, one cannot propose that the task requirements, themselves, of explicitly assigning the referent of a pronoun, were automatic in the sense of being "on-line" and, thus, a reflection of initial first-pass processing. However, it may be argued that the process of determining coreference is a natural, "automatic" language process in some sense. With respect to the results of Experiment 1, therefore, it appears that the ability of RBD individuals to use contextual information at the level of the single sentence reflects the notion that the assignment of coreference within the sentence operates at a relatively automatic level. Concerning the use of contextual information across sentence boundaries, however, the issue of whether or not processing was of a more automatic or effortful nature becomes slightly more complicated, though certainly explicable, in terms of the nature of the task and the stimuli used in the present investigation.

Regarding Experiment 2, it should be noted that disambiguation of the pronoun was possible based solely on the information contained in the subordinate clause of the second sentence. Therefore, one might argue that the influence of the information present in the leading sentence was related to the fact that it was automatically available in the processing 
of the second sentence in terms of the establishment of a discourse model but that it did not have to be actively acted upon. The suggestion that a preceding linguistic context may be automatically available for subsequent language processing is based on the findings of Tompkins and Flowers (1987). They found that a linguistic context that favored a certain mood had a facilitative effect on the abilities of RBD patients to judge the mood of a speaker in the production of a neutral phrase presented auditorily. In contrast, however, an incongruent context did not have an adverse effect on their judgments of mood, as it did for normals and LBD subjects. Based on the work of Posner and Snyder (1975), Tompkins and Flowers (1987) noted that while "automatic primes" can produce a facilitative effect, they are not involved in interference effects. Thus, they argued that , based on their results of facilitation for congruent contexts but no interference for incongruent contexts, the preceding contextual information must have been automatically processed. In view of the results of Experiment 2 of the present investigation, therefore, it also seems likely that the information contained in the leading sentence was automatically available. Coupled with the argument that the assignment of coreference is an automatic aspect of language processing, there is good reason to view the processing requirements of the task in Experiment 2 as being relatively automatic. In light of these considerations, therefore, the finding of a context effect by RBD individuals on this task is not surprising. As an aside, it would be interesting to test the effect of an incongruent context on the abilities of RBD patients to assign coreference. If the reasoning of Tompkins and Flowers (1987) is correct, it would be expected that RBD patients would not be negatively influenced by such a context.

It is also worth noting that the stimuli used in Experiment 2 likely promoted automatic processing in another manner as well -- in terms of redundancy of information. Tompkins (1991; Tompkins \& Mateer, 1985) has found evidence to suggest that increased semantic redundancy improves the ability of RBD individuals to infer attitudes and emotions. She pointed out that these findings are consonant with those of Brookshire and 
Nicholas (1984) and Stachowiak et al. (1977) who also invoked the notion of semantic redundancy to explain the relatively good performance of the RBD individuals they tested on tasks of implicit inference and the comprehension of idioms. It should be added that these results are also consistent with those of Rehak et al. (1992). They found that RBD patients were similar to controls in judging story characters' emotions and attributed this finding to the fact that the stories used fully developed the character of one main protagonist, thereby allowing the patients to more fully focus on the feelings of the character. Similarly, the notion of redundancy has also been invoked to explain the positive influence of preceding non-predictive paragraphs on the sentence processing abilities of aphasic individuals (Cannits et al., 1986; Germani \& Pierce, 1992; Hough et al., 1989). Tompkins (1991) has proposed that the benefit of semantic redundancy on language processing may occur because relevant information becomes salient and more readily available and is, thus, more easily integrated. Consequently, redundant semantic information "frees up" more processing resources to deal with the task at hand.

With respect to the stimuli used in Experiment 2, the leading scitences were intentionally constructed to increase redundancy of information and keep the requirements of inferencing to a minimum. In fact, the information provided in the leading sentence was primarily a restatement of the disambiguating information found in the second sentence. Therefore, it seems likely that the redundancy of the information provided in the leading sentence was also beneficial in promoting the use of the contextual information in the resolution of the pronouns by the RBD patients by making available more processing resources.

Concerning Experiment 3, the argument that the task used involved automatic processing is not so easily made as for Experiment 2 . The primary objection involves the fact that the information in the leading sentence had to be acted upon in order to successfully resolve the pronoun. Certainly, to this extent, the processing demands were substantially increased as compared to the demands of the task used in Experiment 2. 
However, given the fact that RBD individuals still exhibited a context effect similar to normals, it seems logical to propose that the demands of the task used in Experiment 3 were still sufficiently automatic as to allow for the use of contextual information by RBD patients. How can this be so?

To begin with, one can still argue that the process of assigning coreference is relatively automatic in language. So the task used in Experiment 3 involved some level of automatic processing. As well, it might be argued that the use of general world knowledge places fewer processing demands on an individual than the use of strictly linguistic contextual knowledge since general world knowledge is arguably well established in an individual's knowledge base and frequently used. Indeed, the results of Chapman and Ulatowska (1989) concerning the abilities of LBD patients to assign coreference suggest this. Recall that they found that the resolution of ambiguous pronouns by aphasic individuals was more difficult when based upon the use of preceding contextual cues as compared to when the resolution could be made based more on the use of general world knowledge. The results of Hier and Kaplan (1980) are also suggestive of this possibility. They found that RBD patients made more errors on questions related to spatial (e.g. "The elephant sat on the mouse. Was the mouse on top?") and passive (e.g. "Mary was telephoned by Fran. Was Fran telephoned?") relationships than to those based on comparative (e.g. "Are trains faster than airplanes?") and temporal ones (e.g. "Does lunch come before dinner?"). The authors suggested that deficits at this level may be related to visuospatial deficits. Interestingly, however, another possible explanation emerges when one considers the kind of information that must be consulted in order to answer each type of question. Both comparative and temporal questions involved the use of general world knowledge. In contrast, the spatial and passive questions required that the subjects specifically consult the previous sentence. Perhaps for these patients, using general world knowledge was easier than actively consulting information in the previous sentence. Finally, it must be stressed that the contextual information provided in the stimuli used in 
Experiment 3 of the present investigation was present in a single sentence. One might argue, therefore, that at this level of minimal discourse one does not have to be selective in encoding relevant information. It is all there for the taking, so to speak. As well, the use of only a single sentence for providing the relevant contextual information obviously reduces demands on short-term memory.

In consideration of these factors it can be argued that, while the demands of the task used in Experiment 3 were likely greater than those for the task used in Experiment 2, they were still sufficiently automatic as to allow for the use of contextual information. It should be stressed that the results of Experiment 3, therefore, suggest that a distinction in terms of, "how effortful is effortful", needs to be further defined in terms of at what point increased processing demands have a negative effect on the abilities of RBD individuals to use contextual information. Certainly, these results suggest that at the level of minimal discourse, RBD individuals may actively use contextual information to resolve pronouns, at least in relation to general world knowledge.

Perhaps effortful processing, in relation to the use of contextual information, relates more to difficulty in using context to impose organizational structure. On such a view, one might predict that difficulty will increase with increased length of discourse since there is more information to manipulate and, consequently, more structure to impose. Thus, in the present investigation, given that the stimuli used consisted of single sentences and two sentence discourses, one can argue that the requirements for imposing structure were minimal. Parenthetically, it is interesting to note that difficulties in the use of contextual information in relation to possible deficits in imposing structure, may help to explain some of the production deficits exhibited by RBD patients. It is well documented that RBD patients often experience difficulty maintaining a topic and have a great propensity for confabulations and tangential remarks (Gardner et al., 1983; Herzyk, 1989; Myers, 1981; Wapner et al., 1981). Certainly, it can be argued that maintenance of a topic requires imposing and deriving organizational structure as a conversation evolves. 
Interestingly, the distinction between automatic and effortful processing may also help to explain some apparent sentence processing difficulties exhibited by RBD patients. It appears that some of the sentence processing deficits demonstrated by RBD patients, such as difficulty solving syllogistic reasoning problems (Caramazza et al., 1976; Grossman, 1982; Joanette et al., 1983) or arranging words in a sentence (Cavalli et al., 1981; Schneiderman \& Saddy, 1988; Ulatowska \& Baker, 1976), may be due to a difficulty in the manipulation of linguistic elements, rather than a parsing deficit per se. In view of the preceding discussion on automatic versus effortful processing, it may, indeed, be the case that the sentence processing difficulties exhibited by RBD patients are more a reflection of the effortful processing required in the tasks used, rather than a reflection of true syntactic deficits.

To summarize, the results of the present investigation support the view of Tompkins and her colleagues (Tompkins, 1990; Tompkins, 1991; Tompkins et al., 1992; Tompkins \& Flowers, 1987) that a proposed impairment in the use of contextual information by RBD patients may be a function of task demands. Recent evidence suggests that RBD patients may be impaired in their ability to engage in effortful processing, and when tasks demand such processing, apparent linguistic difficulties, including deficits in the use of contextual information, may emerge. The results of the current series of studies support this view by demonstrating that, with sufficiently reduced processing demands, RBD individuals exhibit a pattern of response similar to that of NBD controls and LBD patients in using contextual information in pronoun resolution. Of course, it must be emphasized that the tasks used in this investigation were not designed with the purpose of distinguishing between the effects of automatic and effortful processing on the use of contextual information. Thus, although these results are consonant with the findings of Tompkins (1990; 1991) and Tompkins and Flowers (1987) in demonstrating context effects with RBD patients, certainly more controlled studies investigating the relationship between the use of context by $\mathrm{RBD}$ patients and processing demands is 
needed. However, one thing that has become obvious from this investigation is that to posit a deficit in the use of contextual information by RBD patients for the purpose of explaining seemingly varied discourse level deficits is not sufficient.

This is not to say that the discourse level deficits exhibited by RBD patients are not related to problems in using context; they may very well be. However, the challenge now is to more precisely define the extent of this deficit in terms of levels of use (such as in the use of context at the level of the single sentence or at the level of a minimal discourse), as has been attempted in this series of studies, and in terms of processing factors, such as the relationship between the use of contextual information and automatic versus efforful processing requirements. Another interesting research question surfaces if one adheres to the notion that difficulties in the use of contextual information may be a consequence of reduced cognitive/attentional resources and may, therefore emerge when task demands become increasingly more effortful. The question concerns the level at which reduced resources affect the processing of contextual information (Tompkins, 1990). In the case of RBD patients, do reduced attenional and cognitive resources affect the initial encoding of information (Craik and Byrd, 1982), such that there is insufficient contextual information upon which to act? Or, alternatively, does a reduction in processing resources affect the ability to actively use adequately encoded contextual information?

To conclude, this investigation was successful in accomplishing its primary goal -that of qualifying the nature of a proposed deficit in the use of contextual information by RBD patients. The results of all three experiments were consistent in suggesting that, contrary to initial expectations, RBD individuals are influenced and can actively use contextual information in the resolution of pronouns, at least at the level of the single sentence and a minimal discourse. Furthermore, this investigation was successful in demonstrating that the abilities of RBD individuals to assign coreference, an important discourse function, are comparable to those of normal controls. Naturally, the results of this investigation also provoked more questions. In an attempt to explain the findings it 
was suggested, based on the work by Tompkins and her colleagues (Tompkins, 1990; Tompkins, 1991; Tompkins et al., 1992; Tompkins \& Flowers, 1987), that deficits in the use of contextual information by RBD individuals may still emerge as processing demands increase. Thus, future research must address questions concerning whether or not it is, indeed, true that increased processing demands negatively influence the use of contextual information by RBD patients, and, if true, at what level of increased processing do contextual deficits emerge. Possible ways of investigating such a question, within the paradigm of pronoun resolution, include such manipulations as using longer discourses and/or requiring that the disambiguation of a pronoun be made based on relevant information stated in the text (beyond that based on general world knowledge). The one clear implication of the results of this investigation is that it is no longer sufficient to simply propose that discourse level deficits exhibited by RBD individuals are simply a function of an absolute deficit in their ability to use contextual information. 


\section{Footnotes}

1. There were two instances where this pattern was violated due to an oversight in the creation of the stimuli. For the NP1 type verb "approached", under the CC condition the names of the referents were "Anne" and "Gail" in contrast to "Anne" and "Beth" under the NC condition. For the NP1 type verb "won", under the CC-VC condition the subordinate clause consisted of "...because she was a great player", whereas under the NC-VC condition it consisted of "...because she was a good player". Based upon close inspection of the individual item means for these stimuli, it was felt that these minor violations would not influence the overall pattern of results. Therefore, responses to these items were included in the analyses.

2. As a result of some earlier pilot work, the leading sentences for the filler sentences were changed considerably. Initially, there was a mixture of "who", "what", and "where" questions. Many subjects were reporting that they could predict, based on the leading sentence, when a question was to follow and that they were concentrating on the leading sentence only when they expected a question to follow. Obviously, this was counterproductive to the purpose of using filler sentences; thus, the form of the leading sentences for the filler sentences was changed to that of the leading sentences for the experimental sentences.

3. In order to remain consistent with the analyses conducted on the data for Experiment 2, these data were also transformed using the log transformation for the reaction time data and the square root transformation for the accuracy data and then reanalyzed. The results of the reanalysis of the reaction time data were found to be consistent with those reyorted. As well, the results of the reanalysis of the accuracy data were also consistent with those reported with two exceptions -- both the main effect of Context and the interaction Context $\mathrm{x}$ Verb Consistency were found to be significant by subjects only.

4. With reference to Footnote 1, in this study, for the verb "approached", the names of the referents for sentences under all context conditions were "Anne" and "Beth". For the verb 
"won", the subordinate clause of the second sentence for all context conditions was "...because she was a good player".

5. As in Pilot Study 2a, these data were also transformed and reanalyzed. With respect to the reaction time data, the results remained the same as that reported with two exceptions: 1) the interaction Group $x$ Verb Consistency was no longer significant by either subjects or items; 2) pairwise comparison of means under the Context condition using Newman-Keuls procedure found that reaction times to CU sentences were significantly faster than to NC sentences, but by subjects only. The reanalysis of the accuracy data produced results consistent with those reported.

6. Hirst \& Brill (1980) used two sets of stimuli, with one set presented to half of the subjects and the other set presented to the other half of the subjects. The second set of stimuli was a permuted version of the first, in which the first and second clauses were exchanged, except for the names. According to the authors, this was done to control for the possibility of a response bias. However, this control was judged to be unnecessary and was, therefore, excluded from the present experiment. It was felt that simply having both John and Henry appear an equal number of times in both referent positions and by having the preferred referent in both positions an equal number of times was sufficient to control for the possibility of a response bias. Thus, only the first set of stimuli was used in this experiment.

7. Although with these stimuli there is no true correct referent; the preferred referent was designated the correct referent for all conditions except the neutral condition for which there was no preferred referent.

8. In Hirst and Brill (1980) the reaction times to strongly and likely preferred NP1 sentences were combined to form one level of preferred referent - NP1 Preferred; similarly, responses to strongly and likely preferred NP2 sentences were combined to form a second level of preferred referent - NP2 Preferred. As well, Hirst and Brill conducted the analysis of the reaction time data by separately comparing responses to sentences with an 
associated NP1 preference to neutral sentences; and then comparing sentences with an NP2 preference to neutral sentences. As this involved the reanalysis of the same data (i.e. responses to neutral sentences), it was not felt to be statistically sound. Similarly, there appeared to be no sound justification for combining the levels of analysis. Consequently, the analysis reported in the text, in which all five levels are included in the same analysis, was preferred for the purposes of this study. Out of interest, however, the type of analysis conducted by Hirst and Brill was also conducted. The results were similar to those reported. In the analysis of mean response latencies to sentences with NP1 as the preferred referent compared to neutral sentences, once again significant main effects of Group $\left[\mathrm{E} 1(1,38)=15.25, \mathfrak{p}<0.001 ; \mathrm{E} 2(1,22)=143.54, \mathfrak{p}<0.001 ; \min \mathrm{F}^{\prime}(1,46)=13.79\right.$, $p<0.01]$, with the OA group responding more slowly than the YA group overall, and Preferred Referent $\left[\mathrm{E} 1(1,38)=35.82, \mathfrak{p}<0.001 ; \mathrm{E} 2(1,22)=8.71, \mathrm{p}<0.01 ; \min \mathrm{F}^{\prime}(1,33)\right.$ $=7.01, \mathrm{D}<0.05]$, with responses to sentences with NP1 as the preferred referent faster than to Neutral sentences for both groups, were found. The interaction Group $x$ Preferred Referent was not significant by either subjects or items. In the analysis of mean response latencies to sentences with NP2 as the preferred referent compared to neutral sentences, significant main effects of Group $[E 1(1,38)=12.25, p<0.01 ; E 2(1,22)=121.95$, $\left.\mathrm{p}<0.001 ; \min \mathrm{F}^{\prime}(1,45)=11.13, \mathrm{p}<0.01\right]$, with older adults responding more slowly than YA overall, and Preferred Referent $[E 1(1,38)=42.49, \mathrm{p}<0.001 ; \mathrm{E} 2(1,22)=11.13$, $\left.\mathrm{D}<0.01 ; \min \mathrm{F}^{\prime}(1,34)=8.82, \mathrm{p}<0.01\right]$, with responses to sentences with NP2 as the preferred referent faster than to Neutral sentences for both groups, were found. The interaction Group x Preferred Referent was significant by items only $[E 2(1,22)=4.9$, p $<.05]$.

9. As in Pilot Studies $2 a$ and $2 b$, the data in this experiment were transformed and reanalyzed in order to be consistent with the analysis of the data in Experiment 3. The results of this reanalysis were consistent with those reported. 
10. Two other RBD patients were tested but not included in any of the analyses for the following reasons. One of the patients had to constantly be reminded to respond, thus it was felt that her reaction time data would not be an accurate reflection of processing time. The second patient scored poorly (13/20) on the auditory sentence comprehension test of the screening battery (described later in the subject section of Experiment 1).

11. One other LBD patient was tested but not included in the analyses because it was discovered that, in fact, she had experienced multiple strokes. It should also be noted that D.I. had participated as a pilot subject in an earlier version of the task, about 1 year prior to testing on this final version.

12. Two other matched NBD subjects were tested but not included in the analyses because they misunderstood the instructions for the task in Experiment 1. These subjects were subsequently replaced by two other matched NBD subjects.

13. LBD subject I.B., and RBD subjects B.A. and V.C. responded using two fingers of the same hand; LBD subject I.G. used two hands; NBD subject F.U. used her thumb. 14. Due to some confusion in the testing procedure, this task was not administered immediately prior to the experimental task for NBD subject P.K.

15. In one instance, the patient had limb apraxia and could not respond appropriately. In the other instance, the patient reported that he could only see one of the members (i.e. the " $\mathrm{X}$ " or "O") at any given time. It turned out that he could only see items presented in the right visual field, thus evidencing a serious left visual neglect.

16. Due to time constraints, demonstration stimuli were not used in the instructional phase of this task for NBD subject T.A.

17. For LBD subject E.S. and RBD subject F.A. responses to sentences with the verbs "warned", "admired" and "trusted" were also recorded as mechanical errors for the following reasons. The stimuli for verbs "warned" and "admired" were only seen by these two subjects -- they were excluded from the rest of the study as they were not felt to be good stimuli. As well, the stimuli using the verb "trusted" were changed following 
presentation to E.S. and F.A., therefore, responses to these sentences were not included in the analyses for these patients.

18. It should be noted that LBD subject G.W. and RBD subject V.C. served as pilot subjects for an earlier version of the test approximately one year prior to the testing on this final version.

19. The materials from Pilot Study $2 a$ were chosen instead of those from $2 b$ because it was felt that these stimuli were the better of the two. They provided more information and were more coherent, thereby necessitating considerably fewer inferences to make the link between the information provided in the leading sentence and the reason provided in the subordinate clause of the second sentence than those in Pilot Study $2 \mathrm{~b}$.

20. Due to an oversight there was one exception to this. For the verb "won" the second clause in Experiment 1 consisted of "...because she was a good player. In Experiment 2, it consisted of "...because she was a great player.

21. Due to some confusion in the testing procedure, this preliminary task was not presented immediately prior to the experimental task for NBD subjects S.H., M.Q., and J.C.

22. LBD subjects I.B. and I.G. and RBD subject G.M. used a finger on each hand to respond. RBD subject B.A. used 3 fingers of the same hand ("typist style") to respond. 23. RBD subject B.A. was not presented with demonstration stimuli on the computer. 24. As in Experiment 2, LBD subjects I.B. and I.G. used a finger on each hand to respond and RBD subject B.A. used 3 fingers of the same hand ("typist style") to respond. 


\section{References}

Altmann, G. (1987). Modularity and interaction in sentence processing. In J.L. Garfield (Ed.), Modularity in knowledge representation and natural language understanding (pp. 249-257). Cambridge: MIT Press.

Altmann, G.T.M. (1989). Parsing and interpretation: An introduction. Language and Cognitive Processes, 4 (3/4), SI 1-19.

Altmann, G.T.M., Garnham, A., \& Dennis, Y. (1992). Avoiding the garden path: Eye movements in context. Journal of Memory and Language, 31, 685-712.

Altmann, G., \& Steedman, M. (1988). Interaction with context during human sentence processing. Cognition, 30, 191-238.

Archibald, Y.M., \& Wepman, J.M. (1968). Language disturbance and nonverbal cognitive performance in eight patients following injury to the right hemisphere. Brain, 21, 117-130.

Au, T.K.F. (1986). A verb is worth a thousand words: The causes and consequences of interpersonal events implicit in language. Journal of Memory and Language, 25, 104-122.

Basili, A.G., Diggs, C.C., \& Rao, P.R. (1980). Auditory processing of brain-damaged adults under competitive listening conditions. Brain and Language, 2, 362-371.

Belmore, S.M. (1981). Age-related changes in processing explicit and implicit language. Journal of Gerontology, 36, 316-322.

Benton, A. (1985). Visuoperceptual, visuospatial, and visuoconstructive disorders. In K.M. Heilman \& E. Valenstein (Eds.), Clinical neuropsychology (pp. 151-185). New York: Oxford University Press.

Bihrle, A.M., Brownell, H.H., Powelson, J., \& Gardner, H. (1986). Comprehension of humorous and nonhumorous materials by brain-damaged patients. Brain and Cognition, 5, 399-411. 
Bloom, R.L., Borod, J.C., Obler, L.K., \& Gerstman, L.J. (1992). Impact of emotional content on discourse production in patients with unilateral brain damage. Brain and Language, 42, 153-164.

Borod, J.C., Andelman, F., Obler, L.K., Tweedy, J.R., \& Welkowitz, J. (1992). Right hemisphere specialization for the identification of emotional words and sentences: Evidence from stroke patients. Neuropsychologia, 30(9), 827-844.

Bosch, P. (1983). Agreement and anaphora: A study of the role of pronouns in syntax and discourse. London: Academic Press.

Bransford, J.D., \& Johnson, M.K. (1972). Contextual prerequisites for understanding: Some investigations of comprehension and recall. Journal of Verbal Learning and Verbal Behavior, 11, 717-726.

Brookshire, R.H., \& Nicholas, L.E. (1984). Comprehension of directly and indirectly stated main ideas and details in discourse by brain-damaged and non-brain-damaged listeners. Brain and Language, 21, 21-36.

Brown, G., \& Yule, G. (1983). Discourse analysis (pp. 190-222). Cambridge: Cambridge University Press.

Brown, R., \& Fish, D. (1983). The psychological causality implicit in language. Cognition, 14, 233-274.

Brownell, H.H., Carroll, J.J., Rehak, A., \& Wingfield, A. (1992). The use of pronoun anaphora and speaker mood in the interpretation of sonversational utterances by right hemisphere brain-damaged patients. Brain and Language, 43, 121-147.

Brownell, H.H., Potter, H.H., Bihrle, A.M., \& Gardier, H. (1986). Inference deficits in right brain-damaged patients. Brain and Lanquage, 27, 310-327.

Brownell, H.H., Michel, D., Powelson, J., \& Gardner, H. (1983). Surprise but not coherence: Sensitivity to verbal humor in right hemisphere patients. Brain and Language, 18, 20-27. 
Brownell, H.H., Potter, H.H., Michelow, D., \& Gardner, H. (1984). Sensitivity to lexical denotation and connotation in brain-damaged patients: A double dissociation? Brain and Language, 22, 253-265.

Brownell, H.H., Simpson, T.L., Bihrle, A.M., Potter, H.H., \& Gardner, H. (1990). Appreciation of metaphoric alternative word meanings by left and right braindamaged patients. Neuropsychologia, 28(4), 375-383.

Bryan, K.L. (1988). Assessment of language disorders after right hemisphere damage. British Journal of Disorders of Communication, 23, 111-125.

Burgess, C., \& Simpson, G.B. (1988). Cerebral hemispheric mechanisms in the retrieval of ambiguous word meanings. Brain and Language, 33, 86-103.

Burke, D.M., \& Yee, P.L. (1984). Semantic priming during sentence processing by young and older adults. Developmental Psychology, 20(3), 903-910.

Cannito, M.P., Jarecki, J.M., \& Pierce, R.S. (1986). Effects of thematic structure on syntactic comprehension in aphasia. Brain and Language, 27, 38-49.

Caplan, D. (1992). Language: Structure, process, and disorders (pp. 403-441). Cambridge: MIT Press.

Caplan, D., \& Evans, K.L. (1990). The effects of syntactic structure on discourse comprehension in patients with parsing impairments. Brain and Language, 39 , 206-234.

Cappa, S.F., Papagno, C., \& Vallar, G. (1990). Language and verbal memory after right hemispheric stroke: A clinical - CT scan study. Neuropsychologia, 28(5), 503509.

Caramazza, A., Gordon, J., Zurif, E.B., \& DeLuca, D. (1976). Right-hemispheric damage and verbal problem solving behavior. Brain and Language, 3, 41-46.

Caramazza, A., Grober, E., Garvey, C., \& Yates, J. (1977). Comprehension of anaphoric pronouns. Journal of Verbal Learning and Verbal Behavior, 16, 601-609. 
Caramazza, A., \& Zurif, E.B. (1976). Dissociation of algorithmic and heuristic processes in language comprehension: Evidence from aphasia. Brain and Language, 3, 572582.

Cavalli, M., De Renzi, E., Faglioni, P., \& Vitale, A. (1981). Impairment of right braindamaged patients on a linguistic cognitive task. Cortex, 17, 545-556.

Chapman, S., \& Ulatowska, H.K. (1989). Discourse in aphasia: Integration deficits in processing reference. Brain and Language, 36, 651-668.

Cicone, M., Wapner, W., \& Gardner, H. (1980). Sensitivity to emotional expressions and situations in organic patients. Cortex, 16, 145-158.

Clark, A.E., \& Flowers, C.R. (1987). The effect of semantic redundancy on auditory comprehension in aphasia. In R.H. Brookshire (Ed.), Clinical aphasiology: Proceedings of the conference (pp. 174-179). Minneapolis: BRK Publishers.

Clifton, C., \& Ferreira, F. (1987). Modularity in sentence comprehension. In J.L. Garfield (Ed.), Modularity in knowledge representation and natural language understanding(pp. 277-290). Cambridge: MIT Press.

Clifton, C., \& Ferreira, F. (1989). Ambiguity in context. Language and Cognitive Processes, 4 (3/4), SI 77-103.

Cohen, G. (1979). Language comprehension in old age. Cognitive Psychology, 11, 412 429.

Cohen, G. (1981). Inferential reasoning in old age. Cognition, 2, 59-72.

Cohen, G., \& Faulkner, D. (1983). Word recognition: Age differences in contextual facilitation effects. British Journal of Psychology, 74, 239-251.

Cohen, G. \& Faulkner, D. (1984). Memory for text: Some age differences in the nature of the information that is retained after listening to texts. In H. Bouma \& D.G Bouwhuis (Eds.), Attention and performance X: Control of language processes (pp. 501-513). London: Erlbaum. 
Cole, R.A., \& Perfetti, C.A. (1980). Listening for mispronunciations in a children's story: The use of context by children and adults. Journal of Verbal Learning and Verbal Behavior, 19, 297-315.

Cook, N.D. (1989a). Toward a central dogma for psychology. New Ideas in Psychology Z(1), 1-18.

Cook, N.D. (1989b). First things first: A reply to Prather, Gardner and Brownell. New Ideas in Psychology, 7(1), 27-32.

Coslett, H.B., Bowers, D., \& Heilman, K.H. (1987). Reduction in cerebral activation after right hemisphere stroke. Neurology, 37, 957-962.

Coughlan, A.K., \& Warrington, E.K. (1978). Word-comprehension and word-retrieval in patients with localize i : rebral lesions. Brain, 101, 163-185.

Craik, F.I.M., \& Byrd, M. (1982). Aging and cognitive deficits: The role of attentional resources. In F.I.M. Craik \& S. Trehub (Eds.), Aging and cognitive processes (pp. 191-211). New York: Plenum Press.

Crain, S., \& Steedman, M. (1985). On not being led up the garden path: The use of context by the psychological syntax processor. In D. Dowtry, L. Karttunen \& A. Zwicky (Eds.), Natural language parsing: Psychological.computational and theoretical perspectives (pp. 320-358). Cambridge: Cambridge University Press.

Critchley, M. (1962). Speech and speech-loss in relation to the duality of the brain. In V.B. Mountcastle (Ed.), Interhemispheric relations and cerebral dominance (pp. 208-213). Baltimore: The Johns Hopkins Press.

Dagge, M., \& Hartje, W. (1985). Influence of contextual complexity on the processing of cartoons by patients with unilateral lesions. Cortex, 21, 607-616.

Delis, D.C., Wapner, W. Gardner, H., \& Moses, Jr., J.A. (1983). The contribution of the right hemisphere to the organization of paragraphs. Cortex, 19, 43-40. 
Dell, G.S., McKoon, G., \& Ratcliff, R. (1983). The activation of antecedent information during the processing of anaphoric reference in reading. Journal of Verbal Learning and Verbal Behavior, 22, 121-132.

Deloche, G., \& Seron, X. (1981). Sentence understanding and knowledge of the world: Evidences from a sentence-picture matching task performed by aphasic patients. Brain and Language, 14, 57-69.

Diggs, C.C., \& Basili, A. G. (1987). Verbal expression of right cerebrovascular accident patients: Convergent and divergent language. Brain and Language, 30, 130-146.

Dooling, D.J., \& Lachman, R. (1971). Effects of comprehension on retention of prose. Journal of Experimental Psychology,88, 216-222.

Ehrlich, K. (1980). Comprehension of pronouns. Quarterly Journal of Experimental Psychology, 32, 247-255.

Eisenson, J. (1962). Language and intellectual modifications associated with right cerebral damage. Language and Speech, 5, 49-53.

Ferreira, F., \& Clifton, C. (1986). The independence of syntactic processing. Journal of Memory and Language, 25, 348-368.

Fiedler, K., \& Semin, G.R. (1988). On the causal information conveyed by different interpersonal verbs: The role of implicit sentence context. Social Cognition, 6(1), 21-39.

Fodor, J.A. (1983). The modularity of mind: An essay on faculty psychology. Cambridge: MIT Press.

Foldi, N.S. (1987). Appreciation of pragmatic interpretations of indirect commands: Comparison of right and left hernisphere brain-damaged patients. Brain and Language. $31.88-108$.

Foldi, N.S., Cicone, M., \& Gardner, H. (1983). Pragmatic aspects of communication in brain-damaged patients. In S.J. Segalowitz (Ed.), Language functions and brain organization (pp. 51-86). New York: Academic Press. 
Folstein, M.F., Folstein, S.E., \& McHugh, P.R. (1975). "Mini-mental state": A practical method for grading the cognitive state of patients for the clinician. Joumal of Psychiatric Research, 12, 189-198.

Forster, K.I. (1979). Levels of processing and the structure of the language processor. In W.E. Cooper and E.C.T. Walker (Eds.), Sentence processing: Psycholinguistic studies presented to Merrill Garrett (pp. 27-85). Hillsdale: Lawrence Erlbaum.

Foss, D.J., \& Jenkins, C.M. (1973). Some effects of context on the comprehension of ambiguous sentences. Journal of Verbal Learning and Verbal Behavior, 12, 577 589.

Frazier, L. (1978). On comprehending sentences: Syntactic parsing strategies. Unpublished doctoral dissertation, University of Connecticut.

Frazier, L. (1987). Theories of Sentence Processing. In J.L. Garfield (Ed.), Modularity in knowledge representation and natural language understanding (pp. 291-307). Cambridge: MIT Press.

Frazier, L., \& Rayner, K. (1982). Making and correcting errors during sentence comprehension: Eye movements in the analysis of structurally ambiguous sentences. Cognitive Psychology, 14, 178-210.

Friederici, A.D. (1983). Aphasics' perception of words in sentential context: Some realtime processing evidence. Neuropsychologia, 21(4), 351-358.

Gainotti, G., Caltagirone, C., \& Miceli, G. (1979). Semantic disorders of auditory language comprehension in right brain-damaged patients. Joumal of Psycholinguistic Research, 8(1), 13-20.

Gainotti, G., Caltagirone, C., \& Miceli, G. (1983). Selective impairment of semanticlexical discrimination in right-brain-damaged patients. In E. Perecman (Ed.), Cognitive processing in the right hemisphere (pp. 149-167). New York: Academic Press. 
Gainotti, G., Caltagirone, C., Miceli, G., \& Masullo, C. (1981). Selective semantic-lexical impairment of language comprehension in right-brain-demaged patients. Brain and Language, 13, 201-211.

Gardner, H. (1981). How the split brain gets a joke. Psychology Today, February, 74-76.

Gardner, H., Albert, M.L., \& Weintraub, S. (1975). Comprehending a word: The influence of speed and redundancy on auditory comprehension in aphasia. Cortex, 11, 155-162.

Gardner, H., Brownell, H., Wapner, W., \& Michelow, D. (1983). Missing the point: The role of the right hemisphere in the processing of complex linguistic materials. In $\mathrm{E}$. Perecman (Ed.), Cognitive processing in the right hemisphere, New York: Academic Press, pp. 169-191.

Gardner, H., \& Denes, G. (1973). Connotative judgments by aphasic patients on a pictorial adaptation of the semantic differential. Cortex, 2, 183-196.

Gardner, H., Ling, P.K., Flamm, L., \& Silverman, J. (1975). Comprehension and appreciation of humorous material following brain damage. Brain, 98, 399-412.

Garnham, A., Oakhill, J., \& Cruttenden, H. (1992). The role of implicit causality and gender cue in the interpretation of pronouns. Language and Cognitive Processes, 7(3/4), 231-255.

Garrod, S., \& Sanford, A.J. (1985). On the real-time character of interpretation during reading. Language and Cognitive Processes, 1(1), 43-59.

Garvey, C., \& Caramazza, A. (1974). Implicit causality in verbs. Linguistic Inquiry, 5, 459-464.

Garvey, C., Caramazza, A., \& Yates, J. (1976). Factors influencing assignment of pronoun antecedents. Cognition, 3(3), 227-243.

Gauthier, L., Dehaut, F., \& Joanette, Y. (1989). The Bells Test: A quantitative and qualitative test for visual neglect. Intemational Joumal of Clinical Neuropsychology, 11(2), 49-54. 
Germani, M.J., \& Pierce, R.S. (1992). Contextual influences in reading comprehension in aphasia. Brain and Language, 42, 308-319.

Glosser, G., \& Deser, T. (1990). Patterns of discourse production among neurological patients with fluent language disorders. Brain and Language, 40, 67-88.

Glosser, G., \& Goodglass, H. (1991). Idiosyncratic word associations following right hemisphere damage. Journal of Clinical and Experimental Neuropsychology, 13(5), 703-710.

Grober, E.H., Beardsley, W., \& Caramazza, A. (1978). Parallel function strategy in pronoun assignment. Cognition, 6, 117-133.

Grober, E., \& Kellar, L. (1981). Semantic influences on pronoun assignment in aphasia. Applied Psycholinguistics, 2, 253-268.

Grossman, M. (1982). Reversal operations after brain damage. Brain and Cognition, 1 , 331-359.

Grosz, B.J., Pollack, M.E., \& Sidner, C.L.(1989). Computational models of discourse. In M. Posner, (Ed.), Eoundations of cognitive science Boston: MIT Press. Grosz, B.J., \& Sidner, C.L. (1986). Attention, intentions, and the structure of discourse. Computational Linguistics, 12(3), 175-204.

Halliday, M.A.K, \& Hasan, R. (1976). Cohesion in English. London: Longman Grp. Limited.

Hasher, L., \& Zacks, R.T. (1979). Automatic and effortful processes in memory. Joumal of Experimental Psychology: General, 108, 356-388.

Heeschen, C. (1980). Strategies of decoding actor-object-relations by aphasic patients. Cortex, 16, 5-19.

Herzyk, A. (1989). Recall of verbal texts in patients with unilateral brain damage. Polish Psychological Bulletin, 20(2), 147-152.

Hess, T.M. (1984). Effects of semantically related and unrelated contexts on recognition memory of different-aged adults. Joumal of Gerontology, 39(4), 444-451. 
Hess, T.M. (1985). Aging and context influences on recognition memory for typical and atypical script actions. Developmental Psychology, 21(6), 1139-1151.

Hess, T.M., \& Higgins, J.N. (1983). Context utilization in young and old adults. Joumal of Gerontology, 38(1), 65-71.

Hier, D.B., \& Kaplan, J. (1980). Verbal comprehension deficits after right hemisphere damage. Applied Psycholinguistics, 1(3), 279-294.

Hirst, W., \& Brill, G.A. (1980). Contextual aspects of pronoun assignment. Joumal of Verbal Leaming and Verbal Behavior, 19, 168-175.

Hirst, W., LeDoux, J., \& Stein, S. (1984). Constraints on the processing of indirect speech acts: Evidence from aphasiology. Brain and Language, 23, 26-33.

Hobbs, J.R. (1979). Coherence and coreference. Cognitive Science, 3, 67-90.

Hough, M.S. (1990). Narrative comprehension in adults with right and left hemisphere brain-damage: Theme organization. Brain and Language, 38, 253-277.

Hough, M.S., Pierce, R.S., \& Cannito, M.P. (1989). Contextual influences in aphasia: Effects of predictive versus nonpredictive narratives. Brain and Language, 36 , 325-334.

Huber, W. (1990). Text comprehension and production in aphasia: Analysis in terms of micro- and macroprocessing. In Y. Joanette \& H.H. Brownell (Eds.), Discourse ability and brain damage: Theoretical and empirical perspectives (pp. 154-179). New York: Springer-Veriag.

Huber, W. \& Gleber, J. (1982). Linguistic and nonlinguistic processing of narratives in aphasia. Brain and Language, 16, 1-18.

Hughlings-Jackson, J. (1915). On the nature of the duality of the brain. Brain, 38, 80-86. Joanette, Y., \& Goulet, P. (1990). Narrative discourse in right-brain-damaged righthanders. In Y. Joanette and H.H. Brownell (Eds.), Discoursc ability and brain damage: Theoretical and empirical perspectives. New York: Springer Verlag Publishers. 
Joanette, Y., Goulet, P., \& Hannequin, D. (1990). Right hemisphere and verbal communication. New York: Springer-Verlag.

Joanette, Y., Goulet, P., \& Le Dorze, G. (1988). Impaired word naming in right-braindamaged right-handers: Error types and time-course analyses. Brain and Language 34, 54-64.

Joanette, Y., Goulet, P., Ska, B., \& Nespoulous, J. (1980). Informative content of narrative discourse in right-brain-damaged right-handers. Brain and Language, 29, 81-105.

Joanette, Y., Lecours, A.R., Lepage, Y., \& Lamoureux, M. (1983). Language in righthanders with right-hemisphere lesions: A preliminary study including anatomical, genetic, and social factors. Brain and Language, 20, 217-248.

Kaplan, E., Goodglass, H., \& Weintraub, S. (1983). Boston Naming Test. Philadelphia: Lea \& Febiger.

Kaplan, J.A., Brownell, H.H., Jacobs, J.R., \& Gardner, H. (1990). The effects of right hemisphere damage on the pragmatic interpretation of conversational remarks. Brain and Language, 38, 315-333.

Kintsch, W. \& van Dijk, T.A. (1978). Toward a model of text comprehension and production. Psychological Review, 85(5), 363-394.

Kudo, T. (1984). The effect of semantic plausibility on sentence comprehension in aphasia. Brain and Language, 21, 208-218.

Langacker, R. (1969). On pronominalization and the chain of command. In D.A. Reibel and S.A. Schane (Eds.), Modem studies in English: Readings in transformational grammar (pp. 160-186). Englewood Cliffs, NJ: Prentice Hall.

Lasnik, E. (1976). Remarks on coreference. Linguistic Analysis, 2(1), 1-22.

LeDoux, J.F., Blum, C., \& Hirst, W. (1983). Inferential processing of context: Studies of cognitively impaired subjects. Brain and Language, 19, 216-224. 
Lesser, R. (1974). Verbal comprehension in aphasia: An English version of three Italian tests. Cortex, 10, 247-263.

Lesser, R. (1986). Comprehension of linguistic cohesion after right brain-damage. Paper presented at the 9th European Conference of the International Neuropsychological Society, Veldhoven.

Light, L.L., \& Albertson, S.A. (1988). Comprehension of pragmatic implications in young and older adults. In L.L. Light and D.M. Burke (Eds.), Language, memory and aging (pp. 133-153). New York: Cambridge University Press.

Light, L.L, \& Anderson, P.A. (1985). Working-memory capacity, age, and memory for discourse. Journal of Gerontology, 40(6), 737-747.

Light, L.L., \& Capps, J.L. (1986). Comprehension of pronouns in young and older adults. Developmental Psychology, 22(4), 580-585.

Light, L.L., Valencia-Laver, D., \& Zavis, D. (1991). Instantiation of general terms in young and older adults. Psychology and Aging, 6(3), 337-351.

Light, L.L., Zelinski, E.M., \& Moore, M. (1982). Adult age differences in reasoning from new information. Joumal of Experimental Psychology: Leaming. Memory, and Cognition, 8(5), 435-447.

Lust, B. (1986). Introduction: Anaphora. In B. Lust (Ed.), Studies in the acquisition of anaphora: Defining the constraints (Vol. 1, pp. 9-10). Boston: D.Reidel Publishing Co.

MacDonald, M.C., Pearlmutter, N.J., \& Seidenberg, M.S. (1993). Similarities in lexical and syntactic ambiguity resolution. Paper presented at Sixth Annual CUNY Sentence Processing Conference, Amherst, March.

Madden, D.J. (1988). Adult age differences in the effects of sentence context and stimulus degradation during visual word recognition. Psychology and Aging, 3(2), 167172. 
Marslen-Wilson, W., \& Tyler, L. (1980). The temporal structure of spoken language understanding. Cognition, 8, 1-71.

Marslen-Wilson, W., \& Tyler, L. (1987). Against modularity. In J.L. Garfield (Ed.), Modularity in knowledge representation and natural-language understanding (pp. 37-62). Cambridge: MIT Press.

McDonald, S., \& Wales, R. (1986). An investigation of the ability to process inferences in language following right hemisphere brain damage. Brain and Language, 22, 6880.

McNeil, M.R. \& Kimelman, M.D.Z. (1986). Toward an integrative informationprocessing structure of auditory comprehension and processing in adult aphasia. Seminars in Speech and Language, 1, 123-146.

McNeil, M.R., Odell, K., \& Tseng, C.H. (1991). Toward the integration of resource allocation into a general theory of aphasia. In T. Prescott (Ed.), Clinical aphasiology proceedings, 20 (pp. 21-40). Boston: College Hill Press.

Mitchell, D.C., Corely, M.M.B., \& Garnham, A. (1992). Effects of context in human sentence parsing: Evidence against a discourse-based proposal mechanism. Journal of Experimental Psychology: Leaming. Memory and Cognition, 18(1), 69-88. Molloy, R., Brownell, H.H., \& Gardner, H. (1990). Discourse comprehension by righthemisphere stroke patients: Deficits of prediction and revision. In Y. Joanette and H.H. Brownell (Eds.), Discourse ability and brain damage: Theoretical and empirical perspectives (pp. 113-130). New York: Springer-Verlag Publishers.

Moya, K.L., Benowitz, L.I., Levine, D.N., \& Finklestein, S. (1986). Covariant defects in visuospatial abilities and recall of verbal narrative after right hemisphere stroke. Cortex, 22, 381-397.

Murray, W.S., \& Liversedge, S.P. (1993). Referential context effects on syntactic processing. Paper presented at Sixth Annual CUNY Sentence Processing Conference, Amherst, March. 
Myers, P.S. (1981). Right hemisphere communication impairment. In R. Chapey (Ed.), Language intervention strategies in adult aphasia. 2nd Edition (pp. 444-461). Baltimore: Williams \& Wilkins.

Myers, P.S., \& Linebaugh, C.W. (1981). Comprehension of idiomatic expressions by right-hemisphere-damaged adults. In R.H. Brookshire (Ed.), Clinical aphasiology: Proceedings of the conference (pp. 254-261). Minneapolis: BRK Publishers.

North, A.J., Ulatowska, H.K., Maculuso-Haynes, S., \& Bell, H. (1986). Discourse performance in older adults. International Journal of Aging and Human Development,23(4), 267-283.

Ostrove, J.M., Simpson, T., \& Gardner, H. (1990). Beyond scripts: A note on the capacity of right hemisphere-damaged patients to process social and emotional content. Brain and Cognition 12, 144-154.

Paul, S.T.P., Kellas, G., Martin, M., \& Clark, M.B. (1992). Influence of contextual features on the activation of ambiguous word meanings. ¿ournal of Experimental Byychology: Learning. Memory. \& Cognition, 18(4), 703-717.

Pierce, R.S. (1982). Facilitating the comprehension of syntax in aphasia. Journal of Speech and Hearing Research, 25, 408-413.

Pierce, R.S. (1988). Influence of prior and subsequent context on comprehension in aphasia. Aphasiology, 2(6), 577-582.

Pierce, R.S., \& Beekman, L.A. (1985). Effects of linguistic and extralinguistic context on semantic and syntactic processing in aphasia. Journal of Speech and Hearing. Research 28, 250-254.

Pierce, R.S., \& DeStefano, C.C. (1987). The interactive nature of auditory comprehension in aphasia. Joumal of Communication Disorders, 20, 15-24.

Pierce, R.S., \& Wagner, C.M. (1985). The role of context in facilitating syntactic decoding in aphasia. Joumal of Communication Disorders, 18, 203-213. 
Posner, M.I., \& Snyder, C.R.R. (1975). Attention and cognitive control. In R.L. Solso (Ed.), Information processing and cognition: The Loyola symposium. Hillsdale, NJ: Erlbaum.

Prather, P., Gardner, H., \& Brownell, H.ri. (1989). Providing an anchor for neurolinguistic processing: Should the right hemisphere step forward? A response to Cook. New Ideas in Psychology, 7(1), 19-25.

Pratt, M.W., Boyes, C., Robins, S., \& Manchester, J. (1989). Telling tales: Aging, working memory, and the narrative cohesion of story retellings. Developmental Psychology, 25(4), 628-635.

Rayner, K., Carlson, M., \& Frazier, L. (1983). The interaction of syntax and semantics during sentence processing: Eye movements in the analysis of semantically biased sentences. Joumal of Verbal Learning and Verbal Behavior, 22, 358-374.

Rayner, K., Garrod, S,. \& Perfetti, C.A. (1992). Discourse influences during parsing are delayed. Cognition, 45, 109-139.

Rehak, A., Kaplan, J.A., \& Gardner, H. (1992). Sensitivity to conversational deviance in right-hemisphere-damaged patients. Brain and Language, 42, 203-217.

Rehak, A., Kaplan, J.A., Weylman, S.T., Kelly, B., Brownell, H.H., \& Gardner, H. (1992). Story processing in right-hemisphere brain-damaged patients. Brainand Language, 42, 320-336.

Reinhart, T. (1983). Coreference and bound anaphora: A restatement of the anaphoric questions. Linguistics and Philosophy, 6, 47-88.

Roman, M. Brownell, H.H., Potter, H.H., Seibold, M.S., \& Gardner, H. (1987). Script knowledge in right hemisphere-damaged and in normal elderly aduits. Brain and Language, 31, 151-170.

Sanford, A.J., \& Garrod, S.C. (1989). What, when, and how?: Questions of immediacy in anaphoric reference resolution. Language and Cognitive Processes, 4 (3/4), SI 235-262. 
Schneider, W. (1988). Micro Experimental Laboratory: An integrated system for IBM PC compatibles. Behavior Research Methods. Instruments, and Computers, 20(2), 206-217.

Schneiderman, E.I., Murasugi, K.G., \& Saddy, J.D. (1992). Story arrangement ability in right brain-damaged patients. Brain and Language, 43, 107-120.

Schneiderman, E.I., \& Saddy, J.D. (1988). A linguistic deficit resulting from righthemisphere damage. Brain and Language, 34, 38-53.

Shapiro, L.P., Zurif, E., \& Grimshaw, J. (1987). Sentence processing and the mental representation of verbs. Cognition, 27, 219-246.

Shapiro, L.P., Zurif, E.B., \& Grimshaw, J. (1989). Verb processing during sentence comprehension: Contextual impenetrability. Journal of Psycholinguistic Research, $18(2), 223-243$.

Sheldon, A. (1974). The role of parallel function strategy in the acquisition of relative clauses in English. Joumal of Verbal Leaming and Verbal Behavior, 13, 272-281.

Simon, E. (1979). Depth and elaboration of processing in relation to age. Joumal of Experimental Psychology: Human Leaming and Memory, 5(2), 115-124.

Stachowiak, F.J., Huber, W., Poeck, K., \& Kerschensteiner, M. (1977). Text comprehension in aphasia. Brain and Language, 4, 177-195.

Steedman, M., \& Altmann, G. (1989). Ambiguity in context: A reply. Language and Cognitive Processes, 4 (3/4), SI 105-122.

Stevenson, R.J., \& Vitkovitch, M. (1986). The comprehension of anaphoric relations. Language and Speech, 29, 335-360.

Swinney, D.A. (1979). Lexical access during sentence comprehension: (Re)consideration of context effects. Jourmal of Verbal Leaming_and Verbal Behavior, 18, 645-659.

Swisher, L., \& Samo, M. (1969). Token test scores of three matched patient groups: Left brain-damaged with aphasia; right brain-damaged without aphasia; non-braindamaged. Cortex, 5, 264-273. 
Tabossi, P. (1988). Accessing lexical ambiguity in different types of sentential contexts. Journal of Memory and Language, 27, 324-340.

Tabossi, P., Colombo, L., \& Job, R. (1987). Accessing lexical ambiguity: Effects of context and dominance. Psychological Research, 49, 161-167.

Tanenhaus, M.K., Leiman, J.M., \& Seidenberg, M.S. (1979). Evidence for multiple stages in the processing of ambiguous words in syntactic contexts. Journal of Verbal Leaming and Verbal Behavior, 18, 427-440.

Till, R.E. (1985). Verbatim and inferential memory in young and elderly adults. Joumal of Gerontology, 40, 316-323.

Tompkins, C.A. (1990). Knowledge and strategies for processing lexical metaphor after right or left hemisphere brain damage. Joumal of Speech and Hearing Research, 33, 307-316.

Tompkins, C.A. (1991). Redundancy enhances emotional inferencing by right- and lefthemisphere-damaged adults. Journal of Speech and Hearing Research, 34, 1142 1149.

Tompkins, C.A., Boada, R., \& McGarry, K. (1992). The access and processing of familiar idioms by brain-damaged and normally aging adults. Journal of Speech and Hearing Research, 35, 626-637.

Tompkins, C.A., \& Flowers, C.R. (1987). Contextual mood priming following left and right hemisphere damage. Brain and Cognition, 6, 361-376.

Tompkins, C.A., \& Mateer, C.A. (1985). Right hemisphere appreciation of prosodic and linguistic indications of implicit attitude. Brain and Language, 24, 185-203.

Trueswell, J.C., Tanenhaus, M.K.. \& Garnsey, S.M. (1993). Evidence for the immediate use of local semantic constraints in syntactic ambiguity resolution. Paper presented at Sixth Annual CUNY Sentence Processing Conference, Amherst, March.

Tyler, L. (1985). Real-time comprehension processes in agrammatism: A case study. Brain and Language, 26, 259-275. 
Tyler, L., \& Marslen-Wilson, W.D. (1977). The on-line effects of semantic context on syntactic processing. Journal of Verbal Learning and Verbal Behavior, 16, 683692.

Tyler, L., \& Marslen-Wilson, W. (1982). The resolution of discourse anaphors: Some online studies. Text, 2, 263-291.

Ulatowska, H.K., Allard, L., \& Bond-Chapman (1990). Narrative and procedural discourse in aphasia. In Y. Joanette \& H.H. Brownell (Eds.), Discourse ability and brain damage: Theoretical and empirical perspectives (pp. 180-198). New York: Springer-Verlag.

Ulatowska, H.K., \& Baker, W.D. (1976). A linguistic study of processing strategies in right and left-brain damaged patients. International Journal of Psycholinguistics, 6 , 35-63.

Ulatowska, H.K., \& Bond, S.A. (1983). Aphasia: Discourse considerations. Topics in Language Disorders, 3(4), 21-34.

Ulatowska, H.K., Doyel, A., Freedman-Stern, R., Macaluso-Haynes, S., \& North, A.J. (1983). Production of procedural discourse in aphasia. Brain and Language, 18 , 315-341.

Ulatowska, H.K., Freedman-Stern, R., Weiss-Doyel, A., Macaluso-Haynes, S., \& North, A.J. (1983). Production of narrative discourse in aphasia. Brain and Language, 19, 317-334.

Ulatowska, H.K, Hayashi, M.M., Cannito, M.P., \& Fleming, S.G. (1986). Disruption of reference in aging. Brain and Language, 28, 24-41.

Ulatowska, H.K., North, A.J., \& Macaluso-Haynes, S. (1981). Production of narrative and procedural discourse in aphasia. Brain and Language, 13, 345-371.

van Dijk, T.A., \& Kintsch, W. (1983). Strategies of discourse comprehension. New York: Academic Press. 
Van Lancker, D.R., \& Kempler, D. (1987). Comprehension of familiar phrases by left- but not right-hemisphere damaged patients. Brain and Language, 32, 265-277.

Villardita, C. (1987). Verbal memory and semantic clustering in right brain-damaged patients. Neuropsychologia, 25 (1B), 277-280.

Villardita, C., Grioli, S., \& Quattropani, M.C. (1988). Concreteness/abstractness of stimulus-words and semantic clustering in right brain-damaged patients. Cortex, 24, 563-571.

Vonk, W. (1984). Eye movements during comprehension of pronouns. In A.G. Gale and F. Johnson (Eds.), Theoretical and applied aspects of eye movement research (pp. 203-212). North-Holland: Elsevier Science Publishers.

Vonk, W. (1985a). On the purpose of reading and the immediacy of processing pronouns. In R. Groner, G.W. McConkie and C. Menz (Eds.), Eye movements and human . information processing (pp. 207-215). North-Holland: Elsevier Science Publishers.

Vonk, W. (1985b). The immediacy of inferences in the understanding of pronouns. Rickheit and H. Strohner (Eds.), Inferences in text processing (pp. 205-218). North-Holland: Elsevier Science Publishers.

Waller, M.R., \& Darley, F.L. (1978). The influence of context on the auditory comprehension of paragraphs by aphasic subjects. Journal of Speech and Hearing. Research. 21, 732-745.

Waller, M.R., \& Darley, F.L. (1979). Effect of prestimulation on sentence comprehension by aphasic subjects. Joumal of Communication Disorders, 12, 461-479.

Wapner, W., Hamby, S., \& Gardner, H. (1981). The role of the right hemisphere in the apprehension of complex linguistic materials. Brain and Language, 14, 15-33.

Wechsler, A.F. (1973). The effect of organic brain disease on recall of emotionally charged versus neutral narrative texts. Neurology, 23, 130-135. 
Wechsler, D.W. (1987). Wechsler Memory Scale - Revised. San Antonio: Harcourt, Brace, \& Jovanovich Inc.

Wegner, M.L., Brookshire, R.H., \& Nicholas, L.E. (1984). Comprehension of main ideas and details in coherent and noncoherent discourse by aphasic and nonaphasic listeners. Brain and Language, 21, 37-51.

Weinstein, E.A. (1964). Affections of speech with lesions of the non-dominant hemisphere. Research Publications of the Association for Research in Nervous and Mental Disease, 42, 220-228.

Weylman, S.T., Brownell, H.H., Roman, M., \& Gardner, H. (1989). Appreciation of indirect requests by left- and right-brain-damaged patients: The effects of verbal context and conventionality of wording. Brain and Language, 36, 580-591.

Wilcox, M.J., Davis, G.A., \& Leonard, L.B. (1978). Aphasics' comprehension of contextually conveyed meaning. Brain and Language, 6, 362-377.

Winner, E., \& Gardner, H. (1977). The comprehension of metaphors in brain-damaged patients. Brain, 100, 717-727.

Zacks, R., \& Hasher, L. (1988). Capacity theory and processing of inferences. In L.L. Light and D.M. Burke (Eds.), Language, memory and aging. New York: Cambridge University Press.

Zacks, R., Hasher, L., Doren, B., Hamm, V., \& Attig, M.S. (1987). Encoding and memory of explicit and implicit information. Journal of Gerontology, 42, 418-422.

Zelinski, E.M., \& Miura, S.A. (1990). Anaphor comprehension in younger and older adults. International Joumal of Aging and Human Development, 31(2), 111-134. 


\section{APPENDIX A}

Stimuli for Pilot Study 2a and Experiment 2 (based on Caramazza et al., 1977*)

CC-VC Context consistent-Verb consistent

CC-VI Context consistent-Verb inconsistent

Note: Stimuli under the No context condition are the same VC and VI sentences (with the exceptions noted in Footnote 1) preceded by a string of X's and, therefore will not be repeated here.

\section{NP1 type verbs:}

\section{questioned}

CC-VC Tom was a detective and needed to know what happened to Jack.

Tom questioned Jack because he wanted to learn the truth.

CC-VI Tom knew that Jack was lying and withholding important information.

\section{followed}

Tom questioned Jack because he had not told the truth.

CC-VC Anne was new to the area and did not know where Gail lived.

Anne followed Gail because she did not know the way.

CC-VI Anne believed that Gail had a good sense of direction and knew how to get home.

approached

Anne followed Gail because she knew the way.

CC-VC Anne was in a great mood and wanted to be kind to Gail.

Anne approached Gail because she felt friendly.

CC-VI Anne thought that Gail seemed extremely fun-loving and very kind.

Anne approached Gail because she looked friendly.

sold

CC-VC Tim was broke and required money to pay the bills Mike discovered.

Tim sold the piano to Mike because he needed the cash.

CC-VI Tim knew that Mike was from a very rich family and extremely wealthy.

lied to

Tim sold the piano to Mike because he could pay cash.

CC-VC Ken was sworn to secrecy and was not allowed to tell the news to Pete.

Ken lied to Pete because he could not reveal the truth.

CC-VI Ken discovered that Pete was very confused and not prepared for the facts. Ken lied to Pete because he would not understand the truth. 
lost to

CC-VC Mark had an awful tennis serve and was a terrible player compared to Paul. Mark lost to Paul because he was a poor player.

CC-VI Mark knew that Paul had an excellent tennis serve and was the best player in the game.

confided

Mark lost to Paul because he was a great player.

CC-VC Jane was in big trouble and did not know what to do Gail discovered.

Jane confided in Gail because she needed advice.

CC-VI Jane knew that Gail was extremely wise and had plenty of counselling experience.

confessed

Jane confided in Gail because she could offer advice.

CC-VC

Mark felt extremely guilty for cheating and desired to be pardoned by Paul.

Mark confessed to Paul because he wanted forgiveness.

CC-VI Mark knew that Paul was an understanding priest and would grant absolution. won from

Mark confessed to Paul because he offered forgiveness.

CC-VC Anne had an excellent golf swing and was a fantastic golfer compared to Beth. Anne won the game from Beth because she was a great player.

CC-VI Anne discovered that Beth had a weak golf swing and was the worst golfer at the club.

Anne won the game from Beth because she was a lousy player.

\section{NP2 type verbs:}

trusted

CC-VC Pete believed that Mike was a great lawyer and second to none in the field.

Pete trusted Mike because he was the best in the business.

CC-VI Pete was in big trouble and required a talented lawyer like Mike.

punished

Pete trusted Mike because he needed the best in the business.

CC-VC Liz discovered that Rose was dishonest and had swindled some money.

Liz punished Rose because she admitted to cheating.

CC-VI Liz was very honourable and could not accept dishonesty from Rose.

Liz punished Rose because she disapproved of cheating. 
rewarded

CC-VC Beth knew that Liz always provided a strong effort and worked tirelessly for the company.

Beth rewarded Liz because she was a hard worker.

CC-VI Beth appreciated a strong effort and placed great importance on the good work done by Liz.

criticized

Beth rewarded $\mathrm{Liz}$ because she valued hard workers.

CC-VC John discovered that Bill never helped around the house and was always slacking off.

John criticized Bill because he was lazy.

CC-VI John would not stand for a weak effort and despised Bill slacking off.

feared

John criticized Bill because he hated laziness.

CC-VC Tom knew that Jack was a ruthless businessman and a very strong opponent.

Tom feared Jack because he was more powerful.

CC-VI Tom was vulnerable to attack and a very weak opponent compared to Jack.

envied

Tom feared Jack because he was less powerful.

CC-VC Pam knew that Gail came from a wealthy family and had lots of money.

Pam envied Gail because she was so filthy rich.

CC-VI Pam did not earn very much money and was quite poor compared to Gail.

scolded

Pam envied Gail because she could never be so rich.

CC-VC Beth discovered that Jane was irritating and always whining about something. Beth scolded Jane because she was annoying.

CC-VI Beth was in an extremely bad mood and was very irritated with Jane.

Beth scolded Jane because she was annoyed.

\section{congratulated}

CC-VC Tim knew that Paul was a top athlete and finished first in the race.

Tim congratulated Paul because he was successful.

CC-VI Tim had a high opinion of winners and valued the victory by Paul.

praised

Tim congratulated Paul because he appreciated success.

CC-VC Mike discovered that Ken was a skilled carpenter and did extremely fine work. Mike praised Ken because he did a very good job.

CC-VI Mike was extremely satisfied and content with the work done by Ken.

Mike praised Ken because he was pleased with the job. 


\section{APPENDIX B}

Stimuli for Pilot Study 2b (based on Caramazza et al., 1977*)

CC-VC Context consistent-Verb consistent

CC-VI Context consistent-Verb inconsistent

CU-VC Context unrelated-Verb consistent

CU-VI Context unrelated-Verb inconsistent

Note: Stimuli under the No context condition are the same VC and VI sentences as under $\mathrm{CC}$ and $\mathrm{CU}$ preceded by a string of X's and, therefore, will not be repeated here.

NP1 type verbs:

questioned

CC-VC Tom needed to know what happened Jack thought.

Tom questioned Jack because he wanted to learn the truth.

CC-VI Tom believed that Jack was withholding important information.

Tom questioned Jack because he had not told the truth.

CU-VC Tom went to the grocery store Jack thought.

Tom questioned Jack because he wanted to learn the truth.

CU-VI Tom knew that Jack was a good ball player.

followed

Tom questioned Jack because he had not told the truth.

CC-VC Anne did not know how to get home Gail thought.

Anne followed Gail because she did not know the way.

CC-VI

Anne believed that Gail knew how to get home.

Anne followed Gail because she knew the way.

CU-VC Anne enjoyed the concert Gail thought.

Anne followed Gail because she did not know the way.

CU-VI Anne thought that Gail read mystery novels.

Anne followed Gail because she knew the way.

"Reprinted by permission of the publisher and holder of the copyright, Academic Press, Inc. 
approached

CC-VC Anne was in a great mood Beth discovered.

Anne approached Beth because she felt friendly.

CC-VI Anne thought that Beth was particularly welcoming.

Anne approached Beth because she looked friendly.

CU-VC Anne went shopping Beth thought.

Anne approached Beth because she felt friendly.

CU-VI Anne thought that Beth was a red-head.

Anne approached Beth because she looked friendly.

CC-VC Tim had no money to pay the bills Mike discovered.

Tim sold the piano to Mike because he needed the cash.

CC-VI Tim guessed that Mike was extremely wealthy.

Tim sold the piano to Mike because he could pay cash.

CU-VC Tim was a fantastic ball player Mike believed.

Tim sold the piano to Mike because he needed the cash.

CU-VI Tim thought that Mike was a good cook.

Tim sold the piano to Mike because he could pay cash.

CC-VC Ken was sworn to secrecy Pete thought.

Ken lied to Pete because he could not reveal the truth.

CC-VI Ken agreed that Pete was not ready for the facts.

Ken lied to Pete because he would not understand the truth.

CU-VC Ken was a fantastic cook Pete thought.

Ken lied to Pete because he could not reveal the truth.

CU-VI Ken believed that Pete played tennis.

Ken lied to Pete because he would not understand the truth.

lost to

CC-VC Mark was the worst player on the team Paul thought.

Mark lost to Paul because he was a poor player.

CC-VI Mark knew that Paul was the best player on the team.

Mark lost to Paul because he was a great player.

CU-VC Mark was an art student Paul thought.

Mark lost to Paul because he was a poor player.

CU-VI Mark knew that Paul went to university.

Mark lost to Paul because he was a great player. 
confided in

CC-VC Jane had a serious problem Gail discovered.

Jane confided in Gail because she nesded advice.

CC-VI Jane believed that Gail was good at counselling.

Jane confided in Gail because she could offer advice.

CU-VC Jane was bored with the movie Gail thought.

Jane confided in Gail because she needed advice.

CU-VI Jane knew that Gail liked the colour red.

Jane confided in Gail because she could offer advice.

confessed to

CC-VC Mark felt guilty for stealing money Paul thought.

Mark confessed to Paul because he wanted forgiveness.

CC-VI Mark knew that Paul was an understanding priest. Mark confessed to Paul because he offered forgiveness.

CU-VC Mark went to night school Paul thought. Mark confessed to Paul because he wanted forgiveness.

CU-VI Mark knew that Paul attended university.

won from

CC-VC Anne played cards very well Beth thought.

Anne won the game from Beth because she was a good player.

CC-VI Anne discovered that Beth did not play cards well.

Anne won the game from Beth because she was a lousy player.

CU-VC Anne enjoyed the show Beth thought.

Anne won the game from Beth because she was a good player.

CU-VI Anne knew that Beth lived in the country.

Anne won the game from Beth because she was a lousy player. 
NP2 type verbs:

trusted

CC-VC Pete believed that Mike was a great lawyer.

Pete trusted Mike because he was the best in the business.

CC-VI Pete required a great lawyer Mike discovered.

Pete trusted Mike because he needed the best in the business.

CU-VC Pete knew that Mike owned a boat.

Pete trusted Mike because he was the best in the business.

CU-VI Pete went to the airport Mike thought.

punished

Pete trusted Mike because he needed the best in the business.

CC-VC Liz discovered that Rose had a history of being dishonest.

Liz punished Rose because she admitted to cheating.

CC-VI Liz was very strict Rose discovered.

Liz punished Rose because she disapproved of cheating.

CU-VC Liz knew that Rose had short black hair.

Liz punished Rose because she admitted to cheating.

CU-VI Liz went to the library Rose thought.

rewarded

Liz punished Rose because she disapproved of cheating.

CC-VC Beth knew that Liz was a great employee.

Beth rewarded Liz because she was a hard worker.

CC-VI Beth appreciated dedicated employees Liz discovered.

Beth rewarded Liz because she valued hard workers.

CU-VC Beth knew that Liz had dark hair.

Beth rewarded Liz because she was a hard worker.

CU-VI Beth was very tall Liz thought.

criticized

CC-VC John discovered that Bill never cleaned his room.

John criticized Bill because he was lazy.

CC-VI John despised people slacking off Bill discovered.

John criticized Bill because he hated laziness.

CU-VC John discovered that Bill owned a house. John criticized Bill because he was lazy.

CU-VI John was a talented lawyer Bill thought. John criticized Bill because he hated laziness. 
feared

CC-VC Tom knew that Jack was a strong opponent.

Tom feared Jack because he was more powerful.

CC-VI Tom was weak and vulnerable Jack thought.

Tom feared Jack because he was less powerful.

CU-VC Tom knew that Jack had a beard.

Tom feared Jack because he was more powerful.

CU-VI Tom was a bachelor Jack thought.

Tom feared Jack because he was less powerful.

envied

CC-VC Pam knew that Gail was wealthy

Pam envied Gail because she was so filthy rich.

CC-VI Pam was extremely poor Gail discovered.

Pam envied Gail because she could never be so rich.

CU-VC Pam knew that Gail played the trumpet.

Pam envied Gail because she was so filthy rich.

CU-VI Pam was a swimming instructor Gail thought.

Pam envied Gail because she could never be so rich.

scolded

CC-VC Beth discovered that Jane always whined about something.

Beth scolded Jane because she was annoying.

CC-VI Beth was in an extremely bad mood Jane discovered.

Beth scolded Jane because she was annoyed.

CU-VC Beth knew that Jane was a basketball coach.

Beth scolded Jane because she was annoying.

CU-VI Beth went to the store Jane thought.

congratulated

Beth scolded Jane because she was annoyed.

CC-VC Tim knew that Paul finished first in the race.

Tim congratulated Paul because he was successful.

CC-VI Tim valued excellence Paul discovered.

Tim congratulated Paul because he appreciated success.

CU-VC Tim thought that Paul had blonde hair.

Tim congratulated Paul because he was successful.

CU-VI Tim had blue eyes Paul thought.

Tim congratulated Paul because he appreciated success. 
praised

CC-VC Mike discovered that Ken was a skilled worker.

Mike praised Ken because he did a very good job.

CC-VI Mike was extremely satisfied with the work Ken believed.

Mike praised Ken because he was pleased with the job.

CU-VC Mike knew that Ken owned a motorcycle.

Mike praised Ken because he did a very good job.

CU-VI Mike was a good fisherman Ken thought.

Mike praised Ken because he was pleased with the job. 


\section{Appendix C}

Stimuli for Pilot Study 3 and Experiment 3 (from Hirst \& Brill, 1980*)

NP1-SP NP1 strongly preferred

NP1-LP NP1 likely preferred

NP2-SP NP2 strongly preferred

NP2-LP NP2 likely preferred

$\mathrm{N} \quad$ Neutral

Henry went to the party while John stayed at the store.

NP1-SP He danced with some women.

NPI-LP He laughed at the band.

NP2-SP He worked with little enthusiasm.

NP2-LP He chatted with a customer.

N He left after three hours.

Henry went to the party while John drove to the beach.

NP1-SP He stared at the decorations.

NP1-LP He drank up the whiskey.

NP2-SP He sped towards the coast.

NP2-LP He watched for signs.

N He arrived with a girlfriend.

Henry spoke at a meeting while John stayed at the store.

NP1-SP He relied on a few notes.

NP1-LP He argued with a listener.

NP2-SP He looked for a present.

NP2-LP He gossiped with the clerk.

$\mathrm{N} \quad \mathrm{He}$ glanced at the clock.

Henry spoke at a meeting while John drove to the beach.

NP1-SP He lectured on the administration.

NP1-LP He knocked over the water.

NP2-SP He brought along a surfboard.

NP2-LP He stopped at a store.

N He looked toward a friend.

John stood watching while Henry fell down some stairs.

NP1-SP He ran for a doctor.

NP1-LP He laughed with a vengeance.

NP2-SP He tripped over a skate.

NP2-LP He landed on a trampoline.

$\mathrm{N} \quad$ He thought of the future.

*Reprinted by permission of the publisher and holder of the copyright, Academic Press, Inc. 
John stood watching while Henry jumped across a ravine.

NP1-SP He stared back in amazement.

NP1-LP He applauded with little enthusiasm.

NP2-SP He fell into the river.

NP2-LP He messed up the approach.

$\mathrm{N}$

He picked up some money.

John kept fighting while Henry fell down some stairs.

NP1-SP He swung with both fists.

NP1-LP He picked up some gloves.

NP2-SP He rose without a scratch.

NP2-LP He called for some assistance.

$\mathrm{N} \quad \mathrm{He}$ grabbed for a rope.

John kept fighting while Henry jumped across a ravine.

NP1-SP

NP1-LP

NP2-SP

NP2-LP

$\mathrm{N}$

He slipped on a floor.

He punched with great strength.

He landed on a rock.

He relied on a rocket.

He took in some air. 
Appendix D

Verb test: Verbs plus response choices

$\begin{array}{llllll}\text { 1) } & \text { praise } & - & \text { gesture } & - & \text { flatter } \\ \text { 2) } & \text { win } & - & \text { be condemned } & - & \text { be victorious } \\ 3) & \text { question } & - & \text { interrogate } & - & \text { enjoy } \\ \text { 4) } & \text { envy } & - & \text { lust after } & - & \text { comment upon } \\ \text { 5) } & \text { lie } & - & \text { fib } & - & \text { yell } \\ 6) & \text { approach } & - & \text { move towards } & - & \text { place upon } \\ 7) & \text { fear } & - & \text { be proud } & - & \text { be afraid } \\ 8) & \text { scold } & - & \text { exaggerate } & - & \text { reprimand } \\ \text { 9) } & \text { warn } & - & \text { caution } & - & \text { discriminate } \\ 10) & \text { punish } & - & \text { discipline } & - & \text { succeed } \\ \text { 11) } & \text { trust } & - & \text { have concern } & - & \text { have faith } \\ 12) & \text { admire } & - & \text { respect } & - & \text { accuse } \\ 13) & \text { follow } & - & \text { install } & - & \text { trail } \\ 14) & \text { confess } & - & \text { disclose } & - & \text { repeat } \\ 15) & \text { sell } & - & \text { protect } & - & \text { vend } \\ 16) & \text { criticize } & - & \text { find fault } & - & \text { have pity } \\ 17) & \text { lose } & - & \text { be honoured } & - & \text { be defeated } \\ 18) & \text { congratulate } & - & \text { compliment } & - & \text { destroy } \\ 19) & \text { confide } & - & \text { divulge } & - & \text { annoy } \\ \text { 20) } & \text { reward } & - & \text { dislike } & - & \text { recompense }\end{array}$




\section{Appendix E}

Stimuli for Experiment 1 (based on Caramazza et al., 1977*)

$\begin{array}{ll}\text { A-VC } & \text { Ambiguous-Verb consistent } \\ \text { AI-VI } & \text { Ambiguous-Verb inconsistent } \\ \text { U-VC } & \text { Unambiguous-Verb consistent } \\ \text { U-VI } & \text { Unambiguous-Verb inconsistent }\end{array}$

\section{NP1 type verbs}

A-VC Tom questioned Jack because he wanted to learn the truth. A-VI Tom questioned Jack because he had not told the truth.

U-VC Tom questioned Anne because he wanted to learn the truth.

U-VI Anne questioned Tom because he had not told the truth.

A-VC Anne followed Gail because she did not know the way. A-VI Anne followed Gail because she knew the way.

U-VC Anne followed Pete because she did not know the way.

U-VI Pete followed Anne because she knew the way.

A-VC Anne approached Beth because she felt friendly.

A-VI Anne approached Beth because she looked friendly.

U-VC Anne approached Tom because she felt friendly.

U-VI Tom approached Anne because she looked friendly.

A-VC Tim sold the piano to Mike because he needed the cash.

A-VI Tim sold the piano to Mike because he could pay cash.

U-VC Tim sold the piano to Pam because he needed the cash.

U-VI Pam sold the piano to Tim because he could pay cash.

A-VC Ken lied to Pete because he could not reveal the truth.

A-VI Ken lied to Pete because he would not understand the truth.

U-VC Ken lied to Jane because he could not reveal the truth.

U-VI Jane lied to Ken because he would not understand the truth.

A-VC Mark lost to Paul because he was a poor player.

A-VI Mark lost to Paul because he was a great player.

U-VC Mark lost to Beth because he was a poor player.

U-VI Beth lost to Mark because he was a great player.

A-VC Jane confided in Gail because she needed advice.

A-VI Jane confided in Gail because she could offer advice.

U-VC Jane confided in Pete because she needed advice.

U-VI Pete confided in Jane because she could offer advice.

A-VC Mark confessed to Paul because he wanted forgiveness.

A-VI Mark confessed to Paul because he offered forgiveness.

U-VC Mark confessed to Liz because he wanted forgiveness.

U.VI Liz confessed to Mark because he offered forgiveness.

* Reprinted by permission of the publisber and holder of the copyright. Academic Press, Inc. 
A-VC Anne won the game from Beth because she was a good player. A-VI Anne won the game from Beth because she was a lousy player. U-VC Anne won the game from Tim because she was a good player. U-VI Tim won the game from Anne because she was a lousy player.

\section{NP2 type verbs}

A-VC Pete trusted Mike because he was the best in the business.

A-VI Pete trusted Mike because he needed the best in the business.

U-VC Anne trusted Pete because he was the best in the business.

U-VI

Pete trusted Anne because he needed the best in the business.

A-VC Liz punished Rose because she admitted to cheating.

A-VI

$\mathrm{Liz}$ punished Rose because she disapproved of cheating

U-VC Jack punished Liz because she admitted to cheating.

U-VI Liz punished Jack because she disapproved of cheating.

A-VC Beth rewarded Liz because she was a hard worker.

A-VI

U-VC

Beth rewarded Liz because she valued hard workers.

U-VI

Jack rewarded Beth because she was a hard worker.

A-VC John criticized Bill because he was lazy.

A-VI

John criticized Bill because he hated laziness.

U-VC

Anne criticized John because he was lazy.

U-VI

John criticized Anne because he hated laziness.

A-VC Tom feared Jack because he was more powerful.

A-VI Tom feared Jack because he was less powerful.

U-VC Liz feared Tom because he was more powerful.

U-VI

Tom feared $\mathrm{Liz}$ because he was less powerful.

A-VC Pam envied Gail because she was so filthy rich.

A-VI

U-VC

Pam envied Gail because she could never be so rich.

U-VI

Ken envied Pam because she was so filthy rich.

Pam envied Ken because she could never be so rich.

A-VC Beth scolded Jane because she was annoying.

A-VI

Beth scolded Jane because she was annoyed.

U-VC

U-VI

Jack scolded Beth because she was annoying.

A-VC Tim congratulated Paul because he was successful.

A-VI

U-VC

Tim congratulated Paul because he appreciated success.

U-VI

Gail congratulated Tim because he was successful.

Tim congratulated Gail because he appreciated success.

A-VC Mike praised Ken because he did a very good job.

A-VI Mike praised Ken because he was pleased with the job.

U-VC Anne praised Mike because he did a very good job.

U-VI Mike praised Anne because he was pleased with the job. 